\title{
Application of Global Search Methods to Materials Prediction and Design
}

Adam J. Payne

West Virginia University, apayne9@mix.wvu.edu

Follow this and additional works at: https://researchrepository.wvu.edu/etd

Part of the Condensed Matter Physics Commons

\section{Recommended Citation}

Payne, Adam J., "Application of Global Search Methods to Materials Prediction and Design" (2019). Graduate Theses, Dissertations, and Problem Reports. 7377.

https://researchrepository.wvu.edu/etd/7377

This Dissertation is protected by copyright and/or related rights. It has been brought to you by the The Research Repository @ WVU with permission from the rights-holder(s). You are free to use this Dissertation in any way that is permitted by the copyright and related rights legislation that applies to your use. For other uses you must obtain permission from the rights-holder(s) directly, unless additional rights are indicated by a Creative Commons license in the record and/ or on the work itself. This Dissertation has been accepted for inclusion in WVU Graduate Theses, Dissertations, and Problem Reports collection by an authorized administrator of The Research Repository @ WVU.

For more information, please contact researchrepository@mail.wvu.edu. 
Application of Global Search Methods to Materials Prediction and Design

\author{
Adam J. Payne \\ Dissertation submitted \\ to the Eberly College of Arts and Sciences \\ at West Virginia University \\ in partial fulfillment of the requirements for the degree of
}

Ph.D. in

Physics

\author{
Aldo H. Romero, Ph.D., Chair \\ Edward Flagg,Ph.D \\ Mikel Holcomb,Ph.D \\ Lian Li,Ph.D \\ Terence Musho,Ph.D \\ Tudor Stanescu,Ph.D \\ Department of Physics and Astronomy
}

Morgantown, West Virginia

2019

Keywords: structural search, density functional theory, metaheuristics,

firefly algorithm, noncollinear magnetism, orbital occupation, computational physics, condensed matter, NiTi, auxetic, global search, materials prediction, materials design

Copyright 2019 Adam J Payne 


\begin{abstract}
Application of Global Search Methods to Materials Prediction and Design
\end{abstract}

Adam J. Payne

Due to increased availability and power of computational resources over the past few decades, prediction and design of novel materials using computational methods has become feasible. Simulation of material systems has become vital to the further realization of novel material systems. In order to ascertain physical properties, accurate determination and identification of stable crystalline structures is necessary. Additionally, further identification of novel properties, such as magnetic moments or orbital occupation, is necessary to further realize this goal. Global search methods provide a path to accurate prediction of these

properties. In this dissertation, the Firefly algorithm and minima hopping methods are presented. The Firefly algorithm is applied to two problems:

Magnetic moment orientation optimization and orbital occupation optimization. For the first, three noncollinear systems investigated: $\mathrm{NiF}_{2}$, a weak ferromagnet, and $\mathrm{Mn} 3 \mathrm{Pt}$ and the molecular system $\left(\mathrm{Mn}_{I V}\right)_{3} \mathrm{O}_{4} \mathrm{~L}_{4}\left(\mathrm{H}_{2} \mathrm{O}\right)$, where $\mathrm{L}=$ $N, N^{\prime}$-bis(methylene)-Z-1,2-ethenediamine, both of which display frustration.

For the problem of orbital occupation, $\mathrm{UO}_{2}$, an oxide, and $\mathrm{KCoF}_{3}$, a perovskite-like antiferromagnet are investigated to show that FA works equally-well for $d$ and $f$ electron systems. The minima hopping method is applied to the problem of structural search in the binary system NiTi. The convex hull is constructed from this search. From this, results are compared to those previously reported. 


\section{Contents}

1 Introduction 1

2 Theoretical Background 5

2.1 Many-Body Problem: A prelude to DFT . . . . . . . . . . 5 5

2.2 Born-Oppenheimer Approximation . . . . . . . . . . . 7

2.3 Density Functional Theory . . . . . . . . . . . . 8

2.3.1 Thomas-Fermi . . . . . . . . . . . . 9

2.3.2 Hohenberg-Kohn Theorems ............ 11

2.3.3 Kohn-Sham Equation . . . . . . . . . . . . 12

2.3.4 Exchange-Correlation . . . . . . . . . . . 13

2.4 Bloch's Theorem . . . . . . . . . . . . . . . 14

2.5 Electron Correlation - Beyond DFT . . . . . . . . . . 16

2.5.1 Hubbard Model . . . . . . . . . . . . . . . . 17

$2.5 .2 \mathrm{LDA}+\mathrm{U} \ldots \ldots \ldots \ldots$

2.6 Geometric Relaxation . . . . . . . . . . . . . . . . 18

2.6.1 Newtonian Method . . . . . . . . . . . . . . . 19

2.7 Search Methods . . . . . . . . . . . . . . . . . . . . 19

2.7.1 Potential Energy Surface . . . . . . . . . . . 21

2.7.2 Minima Hopping . . . . . . . . . . . . . 22

2.7.3 Metaheuristic Methods . . . . . . . . . . . . 24

2.7.4 Firefly Algorithm .............. 26

3 Magnetic Moment Search 30

$3.1 \mathrm{NiF}_{2} \ldots \ldots \ldots \ldots \ldots \ldots \ldots$

$3.2 \mathrm{MnPt}_{1} \ldots \ldots \ldots \ldots \ldots$

$3.3\left(\mathrm{Mn}_{I V}\right)_{3} \mathrm{O}_{4} \mathrm{~L}_{4}\left(\mathrm{H}_{2} \mathrm{O}\right) \ldots \ldots \ldots \ldots \ldots$

3.4 Chapter Summary . . . . . . . . . . . . . . . . . 49 
4 Orbital Search

4.1 Introduction . . . . . . . . . . . . . . 59

4.2 Methodology ................... 61

4.3 Computational Details . . . . . . . . . . . 663

4.4 Results ..................... 65

$4.4 .1 \quad \mathrm{KCoF}_{3} \ldots \ldots \ldots \ldots 6 \ldots \ldots 65$

$4.4 .2 \quad \mathrm{UO}_{2} \ldots \ldots \ldots \ldots \ldots$

4.5 Chapter Summary . . . . . . . . . . . . 75

5 NiTi 77

5.1 Minima Hopping . . . . . . . . . . . . . . . . 779

5.2 Computational Details . . . . . . . . . . . . 82

5.3 Results And Discussion . . . . . . . . . . . . . . . 84

$5.4 \mathrm{NiTi}_{2} \ldots \ldots \ldots \ldots \ldots 7 . \ldots \ldots$

$5.5 \mathrm{NiTi} \ldots \ldots \ldots \ldots \ldots$

$5.6 \mathrm{Ni}_{3} \mathrm{Ti} \ldots \ldots \ldots \ldots \ldots$

$5.7 \mathrm{Ni}_{5} \mathrm{Ti} \ldots \ldots \ldots \ldots \ldots \ldots$

5.8 Chapter Summary . . . . . . . . . . . . . . . . . . 109

6 Summary and Outlook 


\section{Chapter 1}

\section{Introduction}

Due to increased availability and power of computational resources over the past few decades, prediction and design of novel materials using computational methods has exploded as a field of inquiry. In addition to the development of these resources, advances in Density Functional Theory (DFT), and other software packages based on quantum mechanics has allowed for this field to flourish. Through these developments, simulation of material systems has become vital to the further realization of novel material systems. What previously took years of trial and error can now be done in a fraction of the time computationally. First-principles calculations have been and can be used to accurately predict properties of a vast multitude of material systems, in particular to this work has been its success in prediction of new crystal phases.

However, this is not to say that these methods are complete. While firstprinciples methods based on DFT have been hugely successful in the prediction and design of new crystalline structures, there are limitations. DFT fails dramatically at predicting the properties of any system in which electrons can become localized. However, there have been a number of methods which either build upon DFT or use other methods to treat this problem. These include DFT+U[20], dynamical mean field theory[46], and hybrid functionals[100, 86].

In addition to issues within DFT itself, there are also fundamental issues with predicting novel materials, be it from the standpoint of structural optimization, where the atomic positions are optimized, or from targeting a particular property of interest, such as the band gap, magnetic moments, or orbital occupation. The essence of this problem is the exploration of the Potential Energy Surface (PES), which is a multi-dimensional surface which describes the energy of a system in terms of the system's degrees of freedom. These degrees of freedom are the prop- 
erties of interest for some system, and in regard to past research, these are typically taken to be the positions of atoms in the unit cell of a crystalline material. However, as previously pointed out, the PES does not need to be limited to the atomic positions, but could entail other degrees of freedom, such as magnetic moments, band gaps, or any other physical property that to be optimized. As the PES is a surface which determines the energetics of possible physical configurations of a system's degrees of freedom, solutions which correspond to minima of the surface are energetically favorable solutions. With this in mind, the problem of structural prediction, or that of optimization of any general degrees of freedom reduces to the problem of locating the minima of a multi-dimensional surface, in particular the global minimum, as this would correspond to the most energetically favorable solution, i.e. the ground state configuration of the system. However, this is a highly non-trivial problem, as exhaustive methods to locate the global minimum are computationally infeasible. Thus, methods which are "good enough", meaning that they explore enough of the PES to capture the ground state are necessary to tackle this problem.

In regard to structural search, there have been several methodologies which have been developed and used successfully. These include, but are not limited to genetic algorithms, basin-hopping, minima hopping, simulated annealing, and metaheuristic methods such as particle swarm optimization. While these methods have been wildly successful for the problem of structural prediction, optimization of other degrees of freedom of material systems remains mostly unexplored. In this work, two methods are applied to the problem of optimization of material properties. The first is the minima hopping method (MHM), which has been used successfully in a number of material systems for the problem of structural search. This method is applied to the binary nickel titanium system in order to predict stable crystalline structures for a number of differing stoichiometries.

Nickle titanium alloys, in particular those of near equiatomic composition, are well-known for displaying the properties of shape-memory and the related property of pseudo-elasticity. Discovered in the 1960s, this effect allows for equiatomic NiTi alloys to "remember" their shape after subjected to heating. This effect has its origins in what is known as a martensite transformation. This is a deformation reversing transformation between a low-temperature martensite phase, which has been identified as a monoclinic phase with space group $P 2_{1} / m$ in NiTi, and a high-temperature austenite phase, which is a cubic structure with space group Pm-3m. The martensite phase has a limited ability to undergo limited deformation without breaking its bonds; this consists of rearrangement of atomic planes without causing permanent deformation, and is known as twinning. This 


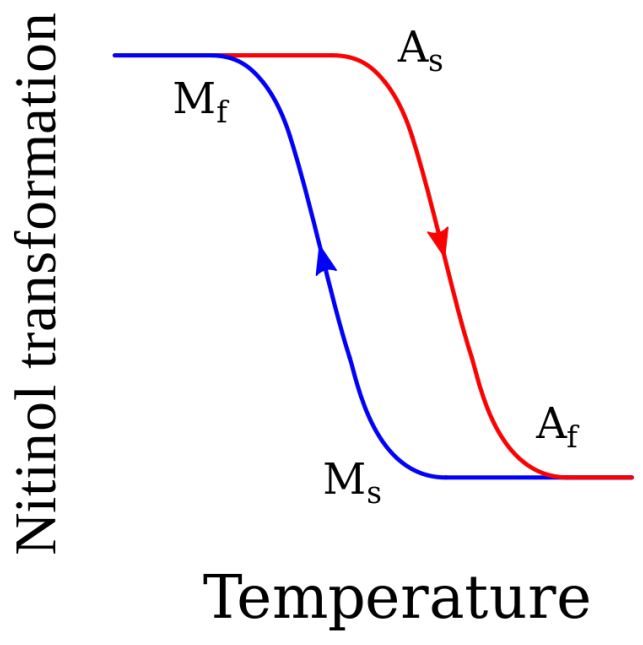

Figure 1.1: Thermal hysteresis in equiatomic NiTi.

twinning is the aforementioned pseudo-elasticity. Upon heating, the system transforms into the austenite phase and this rearrangement is reversed. This is process is the shape-memory effect, and its signature is a thermal hysteresis loop. This hysteresis loop is displayed in Figure 1.1 However, while equiatomic NiTi is known to support this effect, it is unknown if it exists for other compositions of Ni and Ti. Additionally, the phase-space of NiTi is not very well-explored. This motivates the use of search methods to perform a global search of stable crystalline phases across the phase-space of binary NiTi. In this dissertation, the MHM is used to perform this global search. The second is a new method based on the metaheuristic firefly algorithm (FA) which is used not for structural search, but instead applied to the optimization of magnetic moments as well as orbital occupations.

The remainder of this thesis is divided into four chapters. The first chapter consists of presentation of the theoretical background necessary to perform the research presented in this work. In the second chapter, FA is applied to the problem of magnetic moment optimization in magnetic systems. Several systems which have previously been widely studied in the literature are used as test cases to show that FA yields the correct ground states. These include the weak antiferromagnetic $\mathrm{NiF}_{2}$, the frustrated magnetic system $\mathrm{MnPt}$, and a molecular system. It will be seen that the diversity of the magnetic systems tested will demonstrate 
that FA does not depend on system specific details, but can be generalized to any magnetic system.

In the third chapter, FA is applied to the problem of orbital occupation optimization, and the test cases uses include the pervoskite-like $\mathrm{KCoF}_{3}$ as well as $\mathrm{UO}_{2}$, both known for having highly localized d and f-electrons, respectively. From this second case, it will also be shown that FA generates good results which are in agreement with both previous theoretical and experimental works. This will demonstrate like before that FA not only does not depend on system specific details, but also has no dependence on the choice of property to be optimized.

In the fourth chapter, the optimization of a third property is presented. Here, the structural parameters of the binary NiTi system will be optimized using MHM across several differing compositions. The results of the search will be codified through the presentation of the theoretically determined convex hull. Structures on both the convex hull as well as meta-stable states of interest which are within several hundred $\mathrm{meV}$ of the hull structures will be presented.

In the final chapter, the major findings of this work will be briefly summarized in addition to a discussion of the future outlook of research in this area. 


\section{Chapter 2}

\section{Theoretical Background}

In this chapter, the underlying theory which was necessary to perform the research in this work is presented. First, the necessary background to motivate the use of Density Functional Theory (DFT) will be introduced. From there several important approximations and techniques necessary to perform the DFT calculations presented in this work will be discussed. Finally, global search methods will be motivated through a presentation of the potential energy surface (PES), and different methods to perform a global search over physical systems will be presented. In particular, FA and MHM will be focused upon, as these methods are the two which are used in this work.

\subsection{Many-Body Problem: A prelude to DFT}

While presenting DFT is one of the primary purposes of this chapter, first the question of "Why DFT?" must be answered. The underlying problem DFT sought to solve is that of the many-body problem, which ultimately leads to "What is the many-body problem?" and from there "How does DFT solve the many-body problem?"

Everything is comprised of atoms, and those atoms themselves are comprised of a positively-charged nucleus and a negatively-charged electron cloud. In solid state material systems there exists on the order of $10^{23}$ atoms, each with their own cloud of electrons, which interact to form crystalline solids with periodic arrangements of atoms, or amorphous solids with non-periodic arrangements. In both cases, the interactions between electrons in effect determines the particular arrangement of atoms, so understanding the electronic structure of solids is tanta- 
mount. These interactions form the basis of the money-body problem. To solve this, the starting point must be quantum theory, as the electrons and nuclei interact on length scales where quantum mechanics is necessary.

The development of quantum theory in the 1920s yielded the tools necessary to understand electronic structure. Schrödinger, with his work on the equation bearing his name in 1926 provides the mathematical framework to understand quantum systems. This is the famed Schrodinger equation, which is a wave-equation which predicts the time-evolution of of the state of a quantum system.

$$
\hat{H}|\psi\rangle=i \hbar \frac{d}{d t}|\psi\rangle
$$

Here, $\hat{H}$ is the Hamiltonian of the system, $|\psi\rangle$ is the wave-function, $\hbar$ is the reduced Planck's constant. However, it will be more useful to considered the time-independent form of this equation:

$$
\hat{H}|\psi\rangle=E|\psi\rangle
$$

The time-independent form of the Schrödinger equation can be derived with the assumption that the system stays in the same state as time evolves, i.e. $\mid \psi(\mathbf{x}, t\rangle=$ $e^{i E t / \hbar}|\psi(\mathbf{x})\rangle$ The Hamiltonian can be re-written as:

$$
\hat{H}=\hat{T}+\hat{V}
$$

where $\hat{T}$ is the kinetic energy operator and $\hat{V}$ is the potential energy operator. For a solid system comprised of multiple atoms, this can be further re-written in terms of nuclei and electron contributions:

$$
\hat{H}=\hat{T}_{n u c}+\hat{T}_{e l}+\hat{V}_{n u c-n u c} \hat{V}_{e l-e l+\hat{V}_{n u c-e l}}
$$

Since solid systems are comprised of many atoms, each term can be expressed as in terms of the individual nuclei and electrons:

$$
\begin{gathered}
\hat{T}_{n u c}=\frac{-\hbar}{2 M_{I}} \sum_{I=1}^{N} \nabla_{I}^{2} \\
\hat{T}_{e l}=\frac{-\hbar}{2 n_{e}} \sum_{i=1}^{N} \nabla_{i}^{2}
\end{gathered}
$$




$$
\begin{gathered}
\hat{V}_{\text {nuc-nuc }}=\frac{e^{2}}{2} \sum_{I=1}^{N} \sum_{J \neq 1}^{N} \frac{Z_{I} Z_{J}}{\left|\mathbf{R}_{\mathbf{I}}-\mathbf{R}_{\mathbf{J}}\right|} \\
\hat{V}_{\text {nuc-el }}=-\frac{e^{2}}{\sum_{I=1}^{N}} \sum_{i=1}^{N} \frac{Z_{I}}{\left|\mathbf{r}_{\mathbf{i}}-\mathbf{R}_{\mathbf{I}}\right|} \\
\hat{V}_{e l-e l}=\frac{e^{2}}{2} \sum_{i=1}^{N} \sum_{i \neq 1}^{N} \frac{1}{\left|\mathbf{r}_{\mathbf{i}}-\mathbf{r}_{\mathbf{j}}\right|}
\end{gathered}
$$

Since the physical origin of interaction between the nuclei and electrons is electromagnetic in origin, the potential energy terms can be explicitly re-written in terms of Coulomb interactions. In these equations, $\mathbf{R}=\left\{\mathbf{R}_{\mathbf{I}}\right\}$ are the coordinates of the nuclei, $\mathbf{r}=\left\{\mathbf{r}_{\mathbf{i}}\right\}$ are the coordinates of the electrons, $\mathbf{Z}_{\mathbf{I}}$ is the atomic number of the Ith nucleus. $\mathbf{M}_{\mathbf{I}}$ is the mass of the $I$ th nucleus and $\mathbf{m}_{\mathbf{e}}$ is the electron mass. $\mathbf{h}$ is the reduced Planck's constant, with $\hbar=1.054^{-34}$ Js. For a system with this Hamiltonian, the wave-function should only depend upon the electron coordinates, $\mathbf{r}$, and the nuclei coordinates, $\mathbf{R}$ :

$$
\hat{H} \psi_{i}\left(\mathbf{r}, \mathbf{R}=E \psi_{i}(\mathbf{r}, \mathbf{R}\right.
$$

with,

$$
\psi\left(\mathbf{r}, \mathbf{R}=\psi\left(\mathbf{r}_{1}, \mathbf{r}_{2}, \mathbf{r}_{3}, . ., \mathbf{r}_{\mathbf{n}}, \mathbf{R}_{1}, \mathbf{R}_{2}, \mathbf{R}_{3}, \ldots, \mathbf{R}_{\mathbf{N}}\right)\right.
$$

Equation 2.10 is the many-body Schrodinger equation for solid state systems. Since solids contain $10^{23}$ atoms, each atomic site becomes coupled to every other site through the Coulomb interaction. Therefore, this is impossible to compute exactly using current computational resources. To make this problem tractable, a series of physically reasonable approximations must be applied for it to be solved. Next, one of these approximations which is relevant to DFT will be presented.

\subsection{Born-Oppenheimer Approximation}

The Born-Oppenheimer approximation is based around that fact that electrons and much smaller than nuclei, $m_{e}<<M_{I}$. Because of this, electrons will react much faster than the nuclei to any potential. Electron motion is therefore assumed to be instantaneous with respect to motion of the nuclei. This implies that $\hat{T_{n u c}}<<\hat{T_{e l}}$, so the kinetic energy contribution from the nuclei can be ignored; the nuclei can be 
effectively treated as frozen classical particles. This then implies that the Coulomb interaction between nuclei is merely a constant scalar factor, as the coordinates of the nuclei, $\mathbf{R}$ remain fixed. Now the coordinates of the electrons can be described independently of the coordinates of the nuclei; the only remaining effect of the nuclei is the fixed background they created, which is ultimately based upon their geometrical arrangement. From this approximation, it can be seen that the geometrical arrangement of atoms influences the electronic structure of the system. To show this more clearly, equation 2.4 can be re-written:

$$
\hat{H}=\hat{T}_{e l}+\hat{V}_{e l-e l}+\hat{V}_{\text {nuc-el }}
$$

The first two terms are the same for any solid state system. Only the last term varies; since it describes the Coulomb interaction between the nuclei and the electrons it must depend on the geometrical arrangement of the nuclei; differing arrangements of the nuclei yield different solutions to the electronic structure problem. While this term does depend on the coordinates of the nuclei, they remain fixed, so it can be re-written as a function that depends solely on the electronic coordinates:

$$
\hat{V}_{n u c-e l}=\hat{V}_{e x t}=\sum_{i=1}^{n} V_{i}\left(\mathbf{r}_{\mathbf{i}}\right)
$$

This allows us to re-write the Schrodinger equation under this approximation:

$$
\hat{H}_{\text {elec }} \psi_{\text {elec }}(\{\mathbf{r}, \mathbf{R}\})=E(\{\mathbf{r}, \mathbf{R}\}) \psi_{\text {elec }}(\{\mathbf{r}, \mathbf{R}\})
$$

Unlike Equation 2.10, the dependence on the coordinates of the nuclei in Equation 2.14 is parametric. While this has reduced the complexity of the manybody problem, this is still nearly impossible to solve. For a system of $\mathrm{N}$ electrons, there are $3 \mathrm{~N}$ independent variables in the wave-function. DFT provides an alternative approach to this problem.

\subsection{Density Functional Theory}

DFT has been wildly successful as a tool for prediction of properties of material systems. In essence, the idea is to replace the many-body wave-function of $3 \mathrm{~N}$ coordinates for a system with $\mathrm{N}$ electrons with the electronic density, $n(\mathbf{r})$, which 
depends only on three Cartesian coordinates, $\mathbf{r}=(x, y, z)$. This density can be calculated using Born's rule from ordinary quantum mechanics:

$$
n(\mathbf{r})=\left|\psi_{\text {elec }}(\mathbf{R}, \mathbf{r})\right|^{2}=\psi_{\text {elec }}^{*}(\{\mathbf{R}, \mathbf{r}\}) \psi_{\text {elec }}(\{\mathbf{R}, \mathbf{r}\})
$$

Just as in the case of Equation 2.14, the density depends only parametrically on $\mathbf{R}$.

\subsubsection{Thomas-Fermi}

It is of importance to note that the use of the electronic density rather than the wave-function predates DFT by several decades. The Thomas-Fermi model provides a functional form for the kinetic energy of a non-interacting electron gas subjected to an external potential. In a uniform system of non-interacting Fermions, the Fermi momentum, $k_{F}$ can be related to the electronic density by the following expression:

$$
\frac{4 \pi}{3} k_{F}^{3} / \frac{(2 \pi)^{3}}{\Omega}=\frac{N}{2}
$$

Thus,

$$
k_{F}^{3}=3 \pi^{2} \frac{N}{\Omega}=3 \pi^{2} n
$$

where $\Omega$ is the volume occupied in k-space.

The kinetic energy of this system is then given by:

$$
T=\sum_{k<k_{F}} \sum_{\sigma} \frac{\hbar^{2} k^{2}}{2 m_{e}}=\frac{\Omega}{(2 \pi)^{3}} \int_{0}^{k_{F}} 4 \pi k^{2} d k \frac{\hbar^{2} k^{2}}{2 m_{e}}=\frac{\Omega}{\pi^{2}} \frac{\hbar^{2}}{10 m_{e}} k_{F}^{5}
$$

The total number of electrons can be found similarly:

$$
N=\sum_{k<k_{F}} \sum_{\sigma} 1=2 \frac{\Omega}{(2 \pi)^{3}} \int_{0}^{k_{F}} 4 \pi k^{2} d k=\frac{\Omega}{3 \pi^{2}} k_{F}^{3}
$$

Thus, the total kinetic energy per particle (or unit volume) is:

$$
T=N \frac{3}{5} \frac{\hbar^{2} k_{F}^{2}}{2 m_{e}}=\Omega \frac{3}{5} \frac{\hbar^{2} k_{F}^{2}}{2 m_{e}} n
$$


In the semiclassical approximation, a non-uniform system with electronic density $n(\mathbf{r})$ is assumed to have the same functional dependence as above, and:

$$
n(\mathbf{r})=\frac{k_{F}^{3}(\mathbf{r})}{3 \pi^{2}}
$$

Now the kinetic energy becomes a functional of $n(\mathbf{r})$ :

$$
T[n]=\int d^{3} r \frac{3}{5} \frac{\hbar^{2} k_{F}^{2}(\mathbf{r})}{2 m_{e}} n(\mathbf{r})
$$

Lagrange multipliers are used to minimize this under the constrain of constant electron number:

$$
\delta\left(T+\int d^{3} r n(\mathbf{r}) V(\mathbf{r})-\mu \int d^{3} r n(\mathbf{r})\right) / \delta n
$$

This yields the following:

$$
\mu=\frac{\hbar^{2} k_{F}^{2}(\mathbf{r})}{2 m_{e}}+V(\mathbf{r})
$$

Here, $\mu$ is the equilibrium chemical potential. The semiclassical approximation implies that $|\Delta V(\mathbf{r})| / V(\mathbf{r})<<k_{F}(\mathbf{r})$, or that the variation of the external potential is small in comparison to the Fermi wavelength. Eliminating $k_{F}(\mathbf{r})$ in Equations 2.21 and 2.24yields:

$$
n(\mathbf{r})=\frac{1}{3 \pi^{2} \hbar^{3}}\left\{2 m_{e}[\mu-V(\mathbf{r})]\right\}^{3 / 2}
$$

This equation is the main result of Thomas-Fermi. In the interacting case, the potential, $V(\mathbf{r})$ can be replaced with $V_{e f f}=V+V_{H}$, where $V_{H}$ is the Hartree potential, which comes from the Coulomb interaction between electrons:

$$
V_{H}=e^{2} \int d \mathbf{r}^{\prime} \frac{n\left(\mathbf{r}^{\prime}\right)}{\left|\mathbf{r}-\mathbf{r}^{\prime}\right|}
$$

With this, Equation 2.25 becomes:

$$
n(\mathbf{r})=\frac{1}{3 \pi^{2} \hbar^{3}}\left\{2 m_{e}\left[\mu-V(\mathbf{r})-e^{2} \int d \mathbf{r}^{\prime} \frac{n\left(\mathbf{r}^{\prime}\right)}{\left|\mathbf{r}-\mathbf{r}^{\prime}\right|}\right]\right\}^{3 / 2}
$$

Using this in Equation 2.24yields:

$$
\mu=V(\mathbf{r})+e^{2} \int d \mathbf{r}^{\prime} \frac{n\left(\mathbf{r}^{\prime}\right)}{\left|\mathbf{r}-\mathbf{r}^{\prime}\right|}+\frac{\hbar^{2} k_{F}^{2}(\mathbf{r})}{2 m_{e}}
$$


However, this method has several drawbacks. For one, the Pauli exclusion principle is completely ignored, so the exchange energy is not accurately modelled. Secondly, electron correlation effects are not included. While the exchange energy problem was rectified by Dirac in 1928, there still existed the problem of including correlation. Additionally, 1962, Teller showed that Thomas-Fermi is incapable of describing molecular bonding, as the total energy of any molecular system decreases as bond length is increased. The theorems of Hohenberg and Kohn rectify this problem.

\subsubsection{Hohenberg-Kohn Theorems}

DFT is made possible entirely due to the two theorems proposed by Hohenberg and Kohn.

1. Theorem 1: For any interacting system, the external potential, $V_{e x t}(\mathbf{r})$ is a unique functional of the electron density. $n(\mathbf{r})$ in the ground state.

2. Theorem 2: A universal functional of the energy, $E[n(\mathbf{r})]$, can be defined in terms of the density. The exact ground state is the global minima of this functional.

While proofs of these theorems are beyond the scope of this chapter, they imply that any property of a many-body electron system can be determined uniquely as a functional of the ground state density. Now, the many-body Schrodinger equation can be reformulated in terms of the density using the Hamiltonian from Equation 2.12:

$$
E=\left\langle\psi_{e l}\left|\hat{T}_{e l}+\hat{V}_{e l-e l}+\hat{V}_{e x t}\right| \phi_{e l}\right\rangle=\left\langle\psi_{e l}\left|\hat{T}_{e l}+\hat{V}_{e l-e l}\right| \psi_{e l}\right\rangle+\left\langle\psi_{e l}\left|\hat{V}_{e x t}\right| \psi_{e l}\right\rangle
$$

The terms on the right-hand side in terms of the density can be expressed as:

$$
E[n(\mathbf{r})]=T\left[n(\mathbf{r}]+U\left[n(\mathbf{r}]+\int d \mathbf{r} V_{\text {ext }} n(\mathbf{r})\right.\right.
$$

The first-two terms on the right-hand side are universal functionals, as they are the same regardless of the system considered. Only the final term depends on the specifics of the system; from the Born-Oppenheimer approximation is must depend on the specific geometrical arrangement of the nuclei. While the kinetic energy functional, $T[n(\mathbf{r}]$ is relatively straightforward to solve, the evaluation of the electron-electron interaction in the functional $U[n(\mathbf{r}]$ is still challenging. Kohn and Sham were able to solve this problem a year later. 


\subsubsection{Kohn-Sham Equation}

In their groundbreaking work in 1965, Kohn and Sham were able to solve the complicated problem of including the effects of the many-body electron-electron interaction problem. The underlying idea was to replace the complicated manybody system of interacting electrons with the relatively simple problem of a noninteracting system under the effect of a local potential, $V_{\text {eff }}(\mathbf{r})$. With this, the Schrodinger equation can be re-written as:

$$
\left[-\frac{1}{2} \nabla^{2}+V_{e f f}\right] \phi_{i}=\epsilon_{i} \phi_{i}
$$

with,

$$
V_{e f f}=V_{e x t}+V_{H}+V_{x c}
$$

With $V_{\text {ext }}$ arising from the background potential form the nuclei as defined from Equation 2.13, $V_{H}$ is the Hartree potential arising from the Coulomb interaction between electrons as defined in Equation 2.26. $V_{x c}$ is the potential arising from exchange and correlation effects. This term contains all of the missing information form mapping the interacting problem to the non-interacting problem. This equation is known as the Kohn-Sham equation, and in principle, solving it would yield the exact ground state density of the many-body interacting electron system. It must be noted that the states $\phi_{i}$ and energies $\epsilon_{i}$ are not the electronic states and electronic energies, respectively, but are the Kohn-Sham states and corresponding Kohn-Sham energies.

However, there is still one glaring issue with this approach. The exact form of $V_{x c}$ is unknown. This problem will be discussed further in the subsequent section. Assuming that the form of the exchange-correlation is known, one other piece is needed to solve the Kohn-Sham equations. While the Kohn-Sham states are not the electronic states, the total number of electrons in the system still must remain fixed:

$$
N=\int n(\mathbf{r}) d \mathbf{r}
$$

with,

$$
n(\mathbf{r})=\sum_{i=1}\left|\phi_{i}(\mathbf{r})\right|^{2}
$$

With this, the Kohn-Sham equations can be solved, provided $V_{\text {eff }}$ is known. It is of vital importance to emphasize that The equations are solved iteratively, as the potential, $V_{e f f}$ depends on $V_{H}$, which in turn depends on $n(\mathbf{r})$, and knowledge 
of $n(\mathbf{r})$ depends on $V_{\text {eff }}$. To solve the Kohn-Sham equations, the following is performed:

1. Construct $V_{\text {eff }}$ using a trial density, $n_{\text {trial }}(\mathbf{r})$

2. Solve the Kohn-Sham equations to find the Kohn-Sham states, $\phi_{i}$

3. Construct the new density, $n_{K S}(\mathbf{r})$ from the Kohn-Sham states

4. Compare this density, $n_{K S}(\mathbf{r})$, to the original trial density $n_{\text {trial }}(\mathbf{r})$. If they are below some predefined tolerance (ideally zero), stop the calculation, as the ground state density has been found. Otherwise use the new density $n_{K S}(\mathbf{r})$ to construct an updated $V_{e f f}$ and continue the process iteratively until the tolerance is reached.

Thus, the Kohn-Sham equations provide a method to self-consistently construct the ground-state density. The energy corresponding to this density is in principle exact, but the problem of the unknown form of the exchange-correlation terms confounds this. Next, attempts at determining the form of the exchangecorrelation energy functional will be presented.

\subsubsection{Exchange-Correlation}

As previously stated, the exact form of the exchange-correlation functional is not well-defined, but it is of vital importance since it contains the missing information lost from mapping the interacting problem to the non-interacting problem, as it takes into account the exchange energy which arises from the Pauli exclusion principle, as well as the correlation effects between electrons. This term must also take into account the differences in kinetic energy between the non-interacting and interacting cases; as the inclusion of interactions should modify the kinetic energy. As the exact form of this is unknown, there are several popular approximations which are typically used.

\section{Local Density Approximation}

The Local Density Approximation (LDA) was the very first attempt at approximating the exchange-correlation functional, as it still remains as one of the most popular approaches. Much as it's name suggestions, it is local; that is the exchangecorrelation energy, $E_{x c}[n(\mathbf{r})]$, only depends on the density of a homogeneous electron gas at a given point, $r$. Since it assumes the exchange-correlation can 
be approximated as a homogeneous electron-gas, LDA works well for systems in which the electron density is relatively uniform. An important note is that LDA because of this, LDA tends to overestimate exchange and correlation energies. This manifests in the prediction of chemical bonding which is stronger than what is observed in experiment. Thus, mechanical properties like the Bulk modulus are overestimated and bond lengths and strengths are underestimated. The functional form of the exchange-correlation energy under LDA is as follows:

$$
E_{x c}^{L D A}[n(\mathbf{r})]=\int n(\mathbf{r}) \epsilon_{X C}^{L D A}\left[n_{H E G}(\mathbf{r})\right] d \mathbf{r}
$$

where $\epsilon_{X C}^{L D A}\left[n_{H E G}(\mathbf{r})\right]$ is exchange-correlation energy of a particle in a homogeneous electron-gas.

\section{Generalized Gradient Approximation}

The Generalized Gradient Approximation (GGA) is another widely-used approximation to the exchange-correlation energy. It can be thought of as an extension to LDA, as the exchange-correlation energy depends on the local density of a homogeneous electron gas; but it also adds to this a dependence on the gradient of of its local density. The inclusion of this gradient term is to try and capture the effects of in-homogeneity in the electron density. The functional form of the exchange-correlation energy under GGA is as follows:

$$
E_{x c}^{G G A}[n(\mathbf{r})]=\int n(\mathbf{r}) \epsilon_{X C}^{G G A}\left[n_{H E G}(\mathbf{r}), \nabla n(\mathbf{r})\right] d \mathbf{r}
$$

\subsection{Bloch's Theorem}

While in principle everything presented is enough to perform calculations for real solid systems, there is still one problem from a computational standpoint. While the Kohn-Sham equations make the many-body problem tractable in principle, in real systems, there are on the order of $10^{20}$ atoms per cubic millimeter. Similarly to everything presented thus far, this is not computationally possible. However, many solid-state systems are periodic; that is they form crystals. For this reason, the periodic nature of many solid-state systems can be exploited to make computation possible. Bloch's theorem is then used to significantly reduce the total number of degrees of freedom of any periodic solid-state system. From Bloch's 
theorem, an electronic wavefunction, in a periodic potential, $V(\mathbf{r})=V(\mathbf{r}+\mathbf{T})$, $\Psi_{k}^{n}(\mathbf{r})$ can be written in the following form:

$$
\Psi_{k}^{n}(\mathbf{r})=u_{k}^{n}(\mathbf{r}) e^{i \mathbf{k} \cdot \mathbf{r}}
$$

where $u_{k}^{n}(\mathbf{r})$ is a periodic function with the same periodicity as the lattice, $u_{k}^{n}(\mathbf{r})=$ $u_{k}^{n}(\mathbf{r}+\mathbf{T})$,

$\mathrm{k}$

is the crystal momentum, and $e^{i \mathbf{k} \cdot \mathbf{r}}$ is a plane-wave, and $n$ is the band index. Due to Bloch's theorem, wave-functions of this form in a periodic lattice are energy eigenstates. A proof of Bloch's theorem is beyond the scope of this chapter and the interested reader can refer to . Since the system is periodic in real space, it must also be periodic in reciprocal space. The crystal momentum, $\mathrm{k}$ can be can also be written as $\mathbf{k}+\mathbf{K}$, where $\mathbf{K}$ is any reciprocal lattice vector. This implies that any crystal momentum vector which differs from another only by a reciprocal lattice vector is equivalent. This equivalence means that only the set of inequivalent crystal moment vectors needs to be found. The smallest set of inequivalent lattice vectors defines the first Brillouin zone. Therefore, the summation in Equation 2.37 can be restricted to the first Brillioun zone (BZ).

Since $u_{k}^{n}(\mathbf{r})$ is a periodic function with the same period as the periodicity of the crystal, it can be expressed as a Fourier series:

$$
u_{k}^{n}(\mathbf{r})=\frac{1}{\Omega_{\text {cell }}} \sum_{\mathbf{G}} C_{k}^{n}(\mathbf{G}) e^{i \mathbf{G} \cdot r}
$$

where $\Omega_{\text {cell }}$ is the cell volume, $C_{k}^{n}(\mathbf{G})$ are the Fourier coefficients, and $\mathbf{G}$ are the reciprocal lattice vectors. Each one of the Fourier basis functions, $e^{i \mathbf{G} \cdot r}$, represents a plane-wave, propagating perpendicularly to $\mathbf{G}$, with $\mathbf{G} \cdot \mathbf{r}=2 \pi m$, with $m \in Z$. Thus there an an infinite number of allowable reciprocal vectors, $G$. Therefore, in principle, an infinite number of $\mathbf{G}$ vectors are required for maximal accuracy. However, the Fourier coefficients become smaller and smaller as $|\mathbf{G}|^{2}$ becomes larger and larger. In practice, an energy cut-off is defined:

$$
E_{\text {cut }}=\frac{\hbar^{2}}{2 m_{e}}|\mathbf{G}|^{2}
$$

Only plane waves with energies smaller than $E_{c u t}$ are included. In any real calculation, care must be taken to ensure that the cut-off energy is large enough for accurate results. Typically, convergence tests are performed with increasing $E_{c u t}$ 
to determine precisely the value in which further increase yields no numerically relevant difference in predicted properties, which is typically the total energy of the system.

Building upon this, many important properties, such as the charge-density, density of states, and response functions, are defined as integrals over the first BZ. In principle, these are integrated over every infinitesimal point in the first BZ. However, infinite sampling of the space is numerically not possible, so in practice weighted sums are computed. Great care must be taken in selecting a sufficient number of points to yield accurate predictions of these properties. The k-mesh These points are called k-points, and several methods have been developed to determine them. First and foremost, the symmetries of the system can be used to reduce the total number of k-points required; if two k-points can be related through symmetry operations, then determining the results at one k-point automatically yields the result at the other. As the k-mesh is effectively a sampling of a continuous space, this entails that a denser k-mesh will yield better numerical accuracy. However, just as the cut-off energy in Equation 2.39, convergence tests must be performed in order to determine accuracy. As a general rule of thumb, metallic systems require larger k-meshes for convergence than insulating systems.

\subsection{Electron Correlation - Beyond DFT}

A problem with the previously described approximations to the exchange-correlation energy is that both fail to capture the electronic structure of materials with partially filled d and f-electrons; that is they fail to capture the effects of strong electron localization. This particular failure comes from the tendency of LDA/GGA to delocalize electrons due to the loss of self-interaction. The most well-documented failure of DFT is that of Mott insulators. These systems are insulating due to strong Coulomb repulsion between electron, as the contribution to the total energy from this repulsion is larger than the kinetic energy contribution. This forces the electrons into localized atomic-like orbitals. Systems with behavior similar to this are known as strongly-correlated materials, and the correlation energy is formally defined as $E_{c}=E_{\text {exact }}-E_{H F}$, where $E_{H F}$ is the exact value of the Hartree-Fock approximation. Several methods have been developed to correct this problem, e.g. the self-interaction correction (SIC). [83, 31, 20, 91] These include the inclusion of Hubbard U repulsion into the DFT Hamiltonian for some selected orbitals [7], hybrid-functionals [45, 100, 7] or dynamical mean-field theory (DFT+DMFT) [6, 60, 36]. However, other than the inclusion of a Hubbard term to the Hamiltonian, 
each of these methods is computationally expensive. For this reason, the method based on the Hubbard model to include correlation effects is used. In the next section, the Hubbard model is presented.

\subsubsection{Hubbard Model}

One of the more simplistic models use for corrections in strongly-correlated systems is the Hubbard model. In it's simplest form, the Hubbard Hamiltonian is written as:

$$
H_{H u b}=t \sum_{<i, j>, \sigma}\left(c_{i, \sigma}^{\dagger} c^{j, \sigma}+\text { h.c. }\right)+U \sum_{i} n_{i, \uparrow} n_{i, \downarrow}
$$

where $\left\langle i, j>\right.$ denotes the sum is over nearest-neighbor sites, $\sigma$ is the spin, $c_{i, \sigma}^{\dagger}$, $c^{j, \sigma}$, and $n_{i, \sigma}$ and the creation, annihilation and number operators, respectively, H.C. denotes the Hermitian conjugate. The motion of strongly-localized electrons can be described by hopping from one atomic site to it's nearest-neighbor atomic sites. This is the first term in Equation 2.40, and the parameter $t$ is proportional to the bandwidth of valence electron states. Due to strong localization, the Coulomb repulsion can be accounted for between electrons on the same atomic site, meaning that it must be proportional to the product of occupation numbers of atomic states on the same site. The strength of the repulsion is the parameter $U$, this is the second term in Equation 2.40. These two terms, the hopping term (which represents the kinetic energy of the electrons), and the on-site Coulomb repulsion, are the minimal set of parameters necessary to describe the physics of Mott insulators. The insulating nature of these systems comes about when the onsite Coulomb repulsion overcomes the hopping term, that is, when $U>>t$; that is, when the electrons are unable to hop between atomic sties because they lack the energy to overcome the repulsion from other electrons on neighboring atomic sites. This can even occur at half-filling, in which band theory would ordinarily predict metallic behavior.

\subsubsection{LDA+U}

LDA+U is essentially the Hubbard Hamiltonian adapted to DFT, meaning it is correction applied to ordinary DFT functionals (not necessarily LDA as the name suggests). As it is based off of the Hubbard model, it is one of the simplest approaches to correct the description of strongly-correlated materials, and typically 
these are systems in which $\mathrm{d}$ or f-electrons are strongly localized. The total energy of a system within the LDA+U approximation can be written as:

$$
E_{L D A+U}[n(\mathbf{r})]=E_{L D A}[n(\mathbf{r})]+E_{H u b}\left[n\left\{\begin{array}{c}
I \sigma \\
m m^{\prime}
\end{array}\right\}\right]-E_{d c}\left[n\left\{\begin{array}{l}
I \sigma \\
m m^{\prime}
\end{array}\right\}\right]
$$

where the first term is the ordinary energy from DFT, the second is from the Hubbard correction, and the third is a double-counting term. This term is necessary, as it is vital to remove the part of the interaction energy which is accounted for by $E_{H u b}$ from the DFT energy functional, $E_{L D A}$. Due to the approximate nature of the LDA energy functional, the double-counting term cannot be uniquely defined, and there are a multitude of methods to account for it. It must also be stressed that the Hubbard correction is only applied to the localized states of a system. The most general formulation of the Hubbard correction to the LDA energy functional has the following form:

$$
E_{L D A}+\left[\sum_{I} \frac{U^{I}}{2} \sum_{m, \sigma \neq m^{\prime}, \sigma^{\prime}} n_{m}^{I \sigma} n_{m^{\prime}}^{I \sigma^{\prime}}-\frac{U^{I}}{2} n^{I}\left(n^{I}-1\right)\right]
$$

In this equation, $n^{I}=\sum_{m, \sigma} n_{m}^{I \sigma}$, where the index $m$ denotes the localized states on the same atomic site $I$. The second and third terms of the expression denote the Hubbard and double-counting terms, respectively.

\subsection{Geometric Relaxation}

Thus far the methods to treat the electronic density using DFT has been presented, yet methods of structural prediction could not be developed if DFT software does not also include methods to allow relaxation of ionic coordinates and cell parameters. A fully relaxed cell is one where the forces and stresses on the ions in the system vanish. In practice, the forces and stresses must be below some threshold value in order for the cell to be considered relaxed. As knowledge of both forces and stresses are necessary, this must first be calculated. This can be done through the use of the Hellmann-Feynman theorem:

$$
\nabla_{I} \varepsilon_{0}(\mathbf{R})=\frac{\partial}{\partial \mathbf{R}_{\mathbf{I}}}\left\langle\psi_{0}\left|H_{e}(\mathbf{R})\right| \psi_{0}\right\rangle=\left\langle\psi_{0}\left|\nabla_{I} H_{e}(\mathbf{R})\right| \psi_{0}\right\rangle
$$

From this expression, forces acting on the ions can be calculated by finding the expectation values of the gradient of the electronic Hamiltonian in the ground-state. Similarly, the stresses acting on the cell can be calculated in a similar fashion. 
Since the forces and stresses vanish when a cell is relaxed, this is equivalent to saying that the system is in an minimum on the PES. Recall that for the first derivative of the potential energy must vanish in a minimum. For a PES that depends only on ionic coordinates, this is equivalent to the statement that the forces on the ions vanish. Figure 2.1 displays this for a simple 1-D case. However, this can be generalized to multiple dimensions and to any type of generalized coordinates. In fact, the cell shape and the cell volume are examples of non-spatial coordinates that are necessary to fully relax the cell. Relaxation then becomes an iterative process where the forces and stresses are computed in the first step, then the ions are moved along the direction of the forces acting on them in the second. There are several algorithms which are commonly used for this.

\subsubsection{Newtonian Method}

The Newtonian method is the simplest example of a geometrical relaxation algorithm. It consists of the following steps, performed iteratively.

1. Start with an arbitrary point on the PES, $\mathbf{x}=\left(x_{1}, x_{2}, \ldots, x_{N}\right.$

2. Compute both the Hellmann-Feynman forces, $G(\mathbf{x})=\frac{\partial}{\partial \mathbf{x}} U(\mathbf{x})$ and stresses as well as the Hessian, $B$, of the energy

3. Move $\mathrm{x}$ to $\mathrm{x}^{\prime}: \mathrm{x}^{\prime}=\mathrm{x}-B^{-1} g(\mathbf{x})$

4. Repeat steps until the geometry is converged

As this method has a relatively high computational cost, in practice the Hessian matrix is approximated by its largest eigenvalue, $B=\Gamma_{\max }$. Within this approximation, the Newtonian method essentially becomes the steepest descent method, as each step is taken along the direction of the steepest descent.

\subsection{Search Methods}

While first-principles methods such as DFT are useful for determining physical properties of crystalline systems, prediction of new materials is a problem which generally goes beyond the calculation of these properties. The sorts of questions which must be answered are those like "Given a collection of some elements, what combinations form stable crystalline structures?" or "How can we design a 


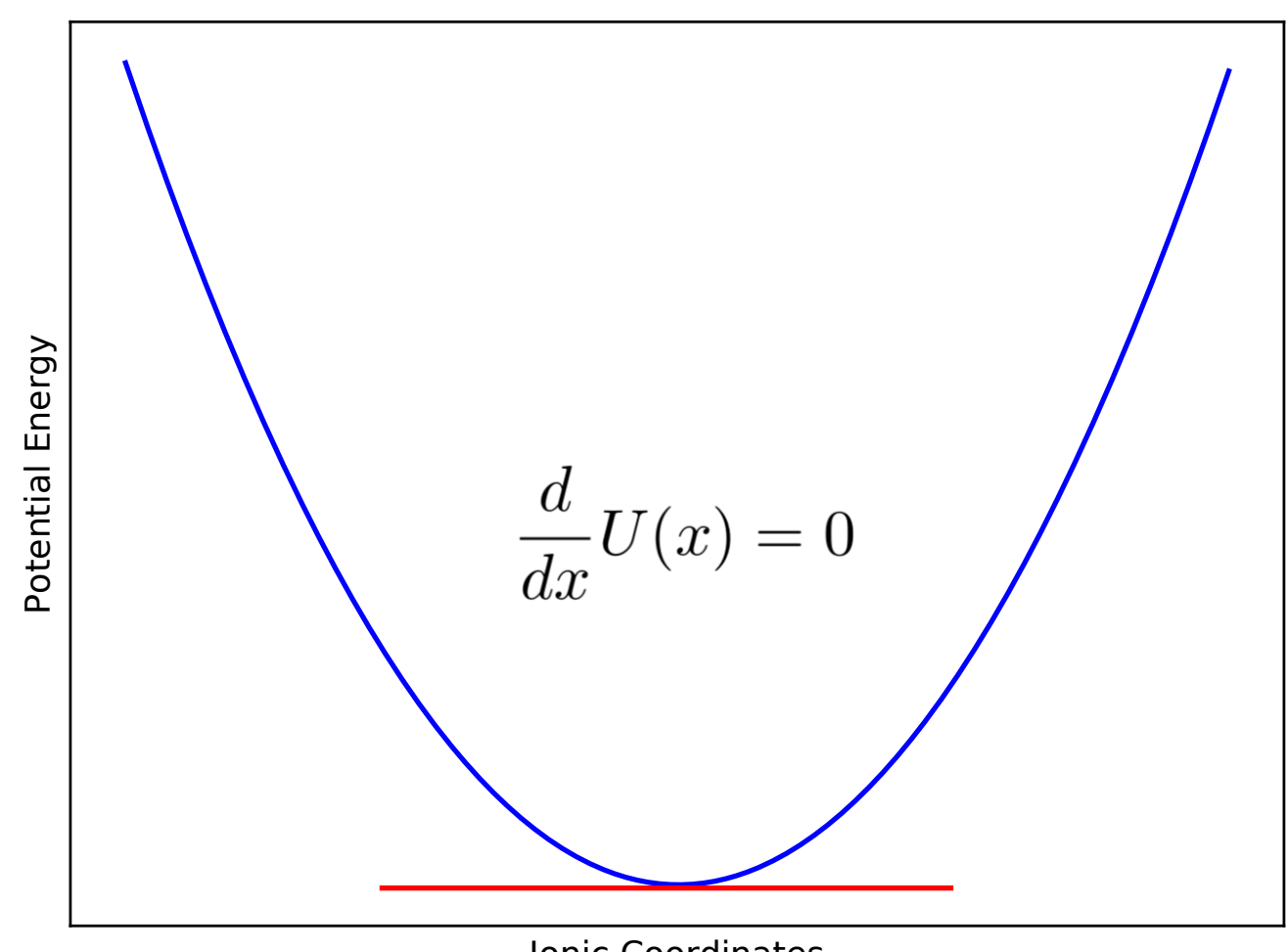

Figure 2.1: 1-D illustration of a minimum on the PES. In this instance, the potential energy, $U \mathbf{x}$ is a function of a single coordinate $x$. At the minimum, this slope of the potential energy function is zero, so the first derivative of this function must vanish. 
new material with useful transport properties?" These are the fundamental problems with materials prediction; given some property, how can optimal solutions be generated? Prior to the advent of cheap and easily available computational resources, this was an arduous task that required many, many years of trial and error. Now, this can be done in a fraction of the time computationally. In general, optimal solutions are those which minimize the formation energy of the system. In the case of structural prediction, such a solution would be the collection of ionic positions and cell parameters which minimize this energy; for magnetic moments with would be the $3 \mathrm{~N}$ coordinates which yield the vectors that describe the $N$ magnetic moments of $N$ magnetic cations which minimize this energy. In addition, there can be multiple optimal solutions. For example, with magnetic frustration there can exist multiple degenerate magnetic moment configurations. More generally, for any material system there will be a collection of $N$ parameters which can vary to generate physically different states. Some of these states can be energy degenerate, and there can also exist several metastable states. While these metastable states will minimize the energy of the system, they do not result in the lowest energy configuration. Taking these different ideas together sets up potential energy surface (PES).

\subsubsection{Potential Energy Surface}

As hinted at above, the PES is the space of potential energies of a collection of atoms or molecules which is a function of their generalized coordinates. These generalized coordinates can be atomic coordinates, bond-lengths, magnetic moments, orbital occupation, or any other degrees of freedom whose variance changes the total energy of the system. This can be defined as:

$$
E=E\left(\mathbf{r}_{1}, \mathbf{r}_{2}, \mathbf{r}_{3}, \ldots, \mathbf{r}_{\mathbf{N}}\right)
$$

where $\mathbf{r}_{\mathbf{i}}$ is the generalized coordinates of atom or molecule $i$. Thus, each point on the PES corresponds to some configuration of the generalized coordinate.

The exploration of the PES in effect is an optimization problem, as solutions which minimize Equation 2.44, i.e. solutions that minimize the total energy are those of interest, as minima on the PES are stable configurations of the generalized coordinates. This is easy to see in the case of $\mathrm{N}$ atoms in a fixed box. In this case the generalized coordinates of the system are just the $3 \mathrm{~N}$ ionic coordinates, and minma on the PES are configurations of these coordinates which satisfy the 
following:

$$
\frac{\partial}{\partial x_{i}} V\left(x_{i}\right)=0, \text { for } \forall i \in 3 N
$$

For atomic coordinates, a minima on the PES is also a solution in which all forces vanish.

In general the PES can depend on any number of generalized coordinates, which means it can be a very complicated function in multiple-dimensions. The multi-dimensional nature is, in essence, the origin of the problem of optimization: Given a multidimensional function, how can minima of the function, both local and global, be found? In principle, it would be ideal if the entirety of the PES could be sampled. Such brute-force methods are computationally impossible, so efficient and accurate techniques to sample the PES have been developed. These include metaheurisitic methods such as genetic algorithms, particle-swarm optimization, and the firefly algorithm, methods based on thermodynamics principles such as simulated annealing and basin hopping, methods based on random sampling such as Monte Carlo and physically-inspired methods such minimum hopping. In the following subsections only minima hopping and metaheuristic methods will be described in more detail detail, as both are the only methods which are relevant to this work.

\subsubsection{Minima Hopping}

One of the more successful methods to sample the PES, which has been widely used for structural-prediction is the Minima Hopping Method (MHM). This method uses both density functional theory (DFT) and short molecular dynamics (MD) simulations to effectively hop around the PES. DFT is used in order to locate local minima. This is done since given any starting point on the PES, which corresponds to both the cell-parameters and ionic coordinates, geometric relaxation can be performed with DFT to find stable configurations of these parameters. Due to equation 2.45, local minima are equivalent to the optimized structures found from geometric relaxation performed using DFT. Using DFT to relax structures to an energy minima is not unique to MHM. In fact, this techniques is common to all the search methods mentioned in the previous section. How each method differs is what is done once a minima is found. In the case of MHM, this is where the MD simulation comes into play. The MHM consists of a two-step process: an inner part which jumps from the current local minima onto a point on the PES in another basin of attraction, with subsequent geometric relaxation, and an outer 


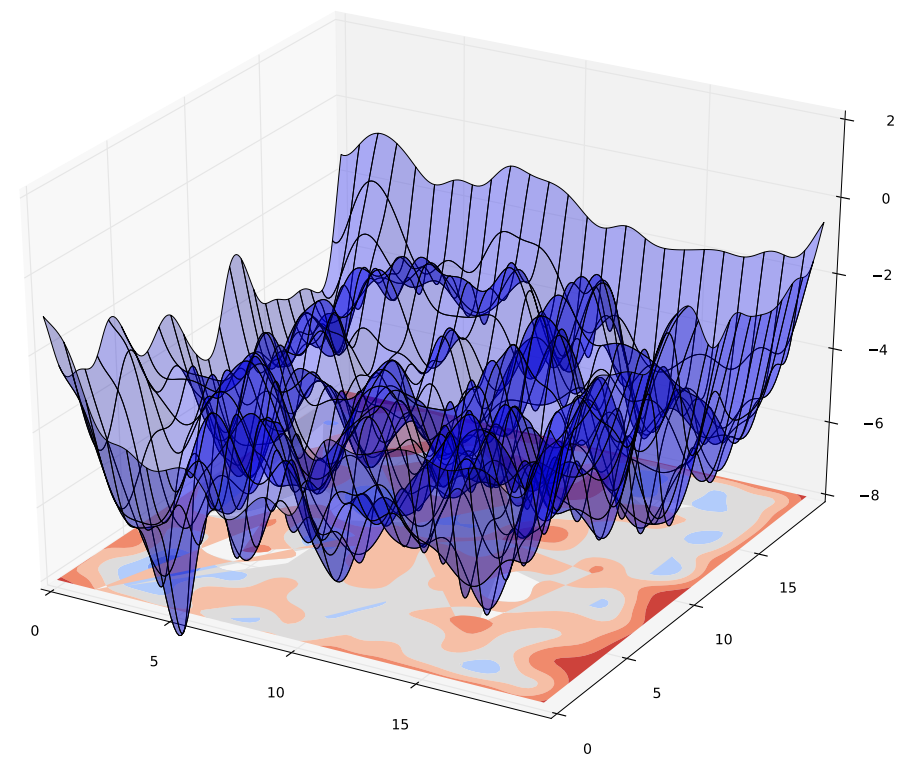

Figure 2.2: Potential energy surface of a system with two generalized coordinates.

part which either accepts or rejects the minima resulting from the geometric relaxation. The jump from the inner part consists of an escape step which is performed with the MD simulation. In this simulation, the atoms have a Boltzmann velocity distribution such that their kinetic energy is equal to $E_{\text {kinetic }}$ This will allow the system cross over energy barriers of height less than $E_{k i n e t i c}$, relative to the current minimum. With this, either $E_{\text {kinetic }}$ is not large enough, and system falls back into the starting local minimum, or it is large enough, and ejection from the current local minima and into another basin of attraction occurs. While is seems that a large value of $E_{\text {kinetic }}$ would be preferable, as it will always result in ejection from the current local minimum, there is a correlation between barrier heights and new minima, due to the Bell-Evans-Polanyi principle. This principle comes from chemistry, in which it states that highly exothermic reactions have low activation energy. It may not seem entirely obvious, but in the structural search context this entails that a new low-energy minimum is more likely found if a low-energy bar- 
rier is crossed rather than a high energy barrier. Figure 2.3 provides an illustration of this principle. Effectively, the smaller the energy difference between two local minima, the larger the energy barrier between the two. With this principle, the MHM method can be used to efficiently search over the low-energy local minima on the PES.

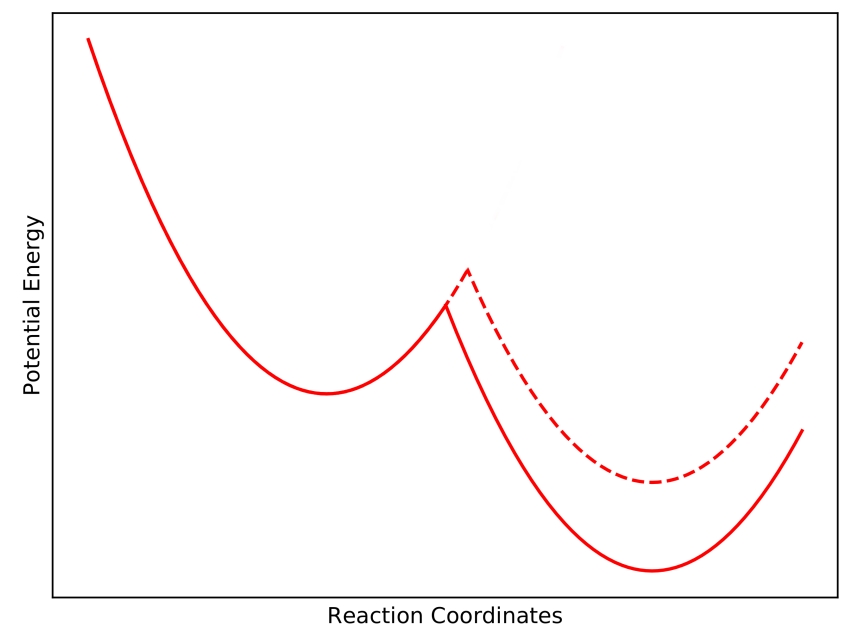

Figure 2.3: Bell-Evans Polyani prinicple. The potential energy of two minima is approximated as two parabolas. The height of the enegry barrier between the two minima depends upon where the two parabolas intersect. The dashed line is a higher-energy local minimum. The height of the energy barrier between the two is raised if the local-minimum on the right is raised.

\subsubsection{Metaheuristic Methods}

Another method to sample the PES is through the use of metaheursitic algorithms. Metaheuristics are a class of computational optimization techniques which seek to generalize heuristic techniques in a problem-independent way. To make this more concrete, first heuristics will be defined and discussed.

Optimization techniques can be roughly divided into two varieties: Exact algorithms and heuristics. Exact algorithms, as their name suggests, are designed to find the exact optimal solution for a given problem in a finite amount of time. There are drawbacks to exact algorithms, however. For the class of NP-hard 
problems, the amount of time to find the optimal solution make scale exponentially with respect to the dimensionality of the problem. Heuristics, on the other hand, are techniques to find optimal solutions to some problem which depend on specifics of the problem at hand. By using system specific details, a good solution to the problem can be found much more quickly than traditional optimization techniques. As a trade-off for an improvement in time, heuristics compromise on accuracy and rigor; in essence the solutions heuristics generate may not be the truly optimal solution, or it may only approximate the optimal solution.

The canonical example is that of the "traveling salesman". The problem is, given $\mathrm{N}$ cities and the distances between pairs of these $\mathrm{N}$ cities, what is the shortest possible route that visits each city and returns to the origin city? Or, more rigorously, given a set of cities $\left\{c_{1}, c_{2}, c_{3}, \ldots, c_{N}\right\}$ and the distance between pairs of cities, $d\left(c_{i}, c_{j}\right)$, what is the ordering $\pi$ which minimizes the following expression:

$$
\sum_{i=1}^{N-1} d\left(c_{\pi(i)}, c_{\pi(i+1)}\right)+d\left(c_{\pi(N)}, c_{\pi(1)}\right)
$$

The most complete way to find the exact solution to this problem would be to use a brute-force method, which would entail considering all possible permutations and then determine which path is the shortest. However, the problem with this approach is that the run time for such an approach is on the order of $O(n !)$, which means this method becomes impractical with as few as twenty cities. The nearest-neighbor algorithm, which is a type of heuristic, allows for this problem to be solved in a fraction of the time. With this method, the salesman will visit the next nearest city, and continues this until all possible cities are visited. Instead of trying to compute every possible path, a single path is generated. There are drawbacks, however. In principle, there is no reason why this method should generate the most optimal solution, and for $\mathrm{N}$ cities randomly located in a plane, the nearest-neighbor algorithm is $25 \%$ longer than the shortest path. Thus, the use of heuristic methods necessitates a trade-off between accuracy and cost.

Metaheuristics are "higher-level" heuristics which attempt to solve the limitations of heuristics in that metaheuristics do not depend on system-specific details. In general, all metaheuristic methods seek the optimal solution to some problem through iteratively attempting to improve a candidate solution with regard to some objective function. The objective function itself is typically the property in which the candidate solution optimizes. From the condensed matter standpoint, the objective function is typically the total energy of the system, and the candidate with 
minimal energy optimizes this objective function, as typically the ground state is what is sought.

\subsubsection{Firefly Algorithm}

The Firefly algorithm (FA) is a population-based metaheuristic which is inspired by the behavior of fireflies. In nature, fireflies are attracted to regions of space with brighter fireflies, and this attraction is modulated by the distance between them. The allows for optimization based on this attraction. While genetic algorithms have been used successfully for structural search [101, 26, 53, 54], for optimization of other physical properties in which energy differences between low-energy states is small $(>1 \mathrm{E}-5 \mathrm{eV})$, it will be seen that FA is better-suited to the task of optimization. This is because the candidate configurations in FA tend to cluster around minima. An objective function, $f(x)$ can be defined and a population of $\mathrm{N}$ candidates $x_{i}$ where $i=1,2,3, \ldots, N$ can be randomly generated. The extremum of $f(x)$ can then be used to define the "brightness" of each candidate. The objective function is then evaluated for each candidate. Candidates are then moved towards one another based on both "brightness" and "distance". The updated candidates are then promoted to the next generation, then continue this entire process iteratively, until a candidate survives for some set number of generations. The evolution of candidate $x_{i}$ can be defined through the following expression:

$$
x_{i}^{t+1}=x_{i}^{t}+\sum_{j} \beta e^{-\gamma r_{i j}^{2}}\left(x_{j}^{t}-x_{i}^{t}\right)+\alpha_{t} \epsilon_{t}
$$

This expression governs how the candidate $i$ in generation $t$ is updated to form candidate $i$ in generation $t+1$. The first term on the right-hand side is the unmodified candidate in generation $i$. Each candidate is generally a vector of some relevant property which is to be optimized. For example, this could be the $3 \mathrm{~N}$ Cartesian coordinates of atoms in a crystal, the $3 \mathrm{~N}$ Cartesian coordinates of magnetic moments for $\mathrm{N}$ magnetic atoms, or the $\mathrm{N} 5 \times 5$ occupation matrices of $\mathrm{N}$ atoms with localized d-orbitals. How candidate $i$ is updated from generation $t$ to generation $t+1$ works as follows. The components of candidate $i$ are modified from generation $t$ to generation $t+1$ by every candidate configuration which is lower in energy. The "weight" of the modification is based upon the distance between candidate $i$ and all candidates $j$ which are lower in energy. Two cases are possible, based on this:

1. Candidate $i$ is the lowest energy candidate. Is is automatically promoted to 
generation $t+1$ either without modification $\left(\alpha_{t}=0\right)$, or with some small amount of randomness $\left(\alpha_{t} \neq 0\right)$. The summation over $j$ is for all candidates $j$ lower in energy than candidate $i$, so the summation term evaluates to zero.

2. There are one or more candidate configurations with energies lower than candidate $i$. In this case, the summation term on the right hand side of Equation 2.47 is evaluated over all candidates which are lower in energy than candidate $i$. This means that candidate $i$ 's magnetic moments will move closer to those which are lower in energy, weighted by the distance between them.

Here this expression be expounded upon in greater detail. First, the third term on the r.h.s. adds randomness to each candidate based on taking values from a Gaussian distribution, $\epsilon_{t}$. The weight of this randomness is determined by $\alpha_{t}$. Since the goal is to locate a minimal energy configurations for some property, then each low-energy candidate found in each generation should be promoted without modification to the next generation. For this reason, $\alpha_{t}=0$ for all performed searches. In the second term, there are two free parameters which must be determined through testing, $\beta$ and $\gamma$. $\beta$ determines the overall strength of the attraction. If $\beta \rightarrow \infty$, then each candidate $i$ is equally attracted to all lower energy candidates $j$, in this case the algorithm becomes a Particle Swarm Optimization (PSO). If $\beta \rightarrow 0$, the algorithm becomes a random walk if $\alpha_{t} \neq 0$. If $\alpha_{t}=0$, then no search is performed since each candidate is automatically updated without modification in each subsequent generation. $\gamma$ is a term in the exponential, which scales the distance between candidates $i$ and $j$; it is the length scale of the considered system. As $\gamma \rightarrow \infty$, the exponential $e^{-\gamma r_{i j}^{2}} \rightarrow 0$, so just as the case where $\beta \rightarrow 0$, the algorithm becomes a random walk. As $\gamma \rightarrow 0$, the weight between different candidates is equal, so candidate $i$ is equally attracted to all lower energy candidates. It is vital that $\gamma$ is chosen with care, as only candidates which are close to each other on the PES should be attracted to one another; candidates outside of the same basin on the PES should not feel attraction to one another. The algorithm itself is an iterative process that is illustrated in Figure 2.4. This flow chart uses magnetic moment optimization as an example, but any property of interest, such as crystal structures or orbital occupations, could be used just as well. The algorithm consists of the following steps:

1. Define an initial population of random candidates.

2. Evaluate the objective function for each candidate. 
3. Compute the distance between candidates.

4. Move candidates towards all candidates which are lower in energy. This movement is defined by Equation 2.47.

5. Compute distances between the candidates, discard identical candidates, replace with random candidates

6. Promote the population to the next generation, continue this process until a candidates survives for a set number of generations.

Here, random means that the property which is being optimized is randomly defined for a collection of different candidates. The objective function evaluation is almost always the evaluation of the energy of each candidate, this is where DFT comes into play. The distance is in effect as measure of similarity between pairs of candidates, and is only the Cartesian distance in instances of structural search. It will be shown in the two test cases of FA where magnetic moments and orbital occupations were optimized that this distance will be defined in terms of angles. In general, this distance Movement means that the property of interest is being modified to be more similar candidates which are lower in energy, with weighting based on the distances between the candidates as in Equation 2.47. Once the movement of candidates occurs, the discarding of identical candidates is based on a pre-defined tolerance in the distance function; if the distance between a pair of candidates is less than this tolerance, they are considered identical and are discarded. This discarding of identical candidates allows for further exploration of the PES, as the number of fireflies per generation remains fixed. These new, random candidates can land in previously unexplored regions of the PES. The number of generations in which a candidate must survive is denoted as the stabilization limit, and it is a free parameter which must be defined for any search performed with FA. 


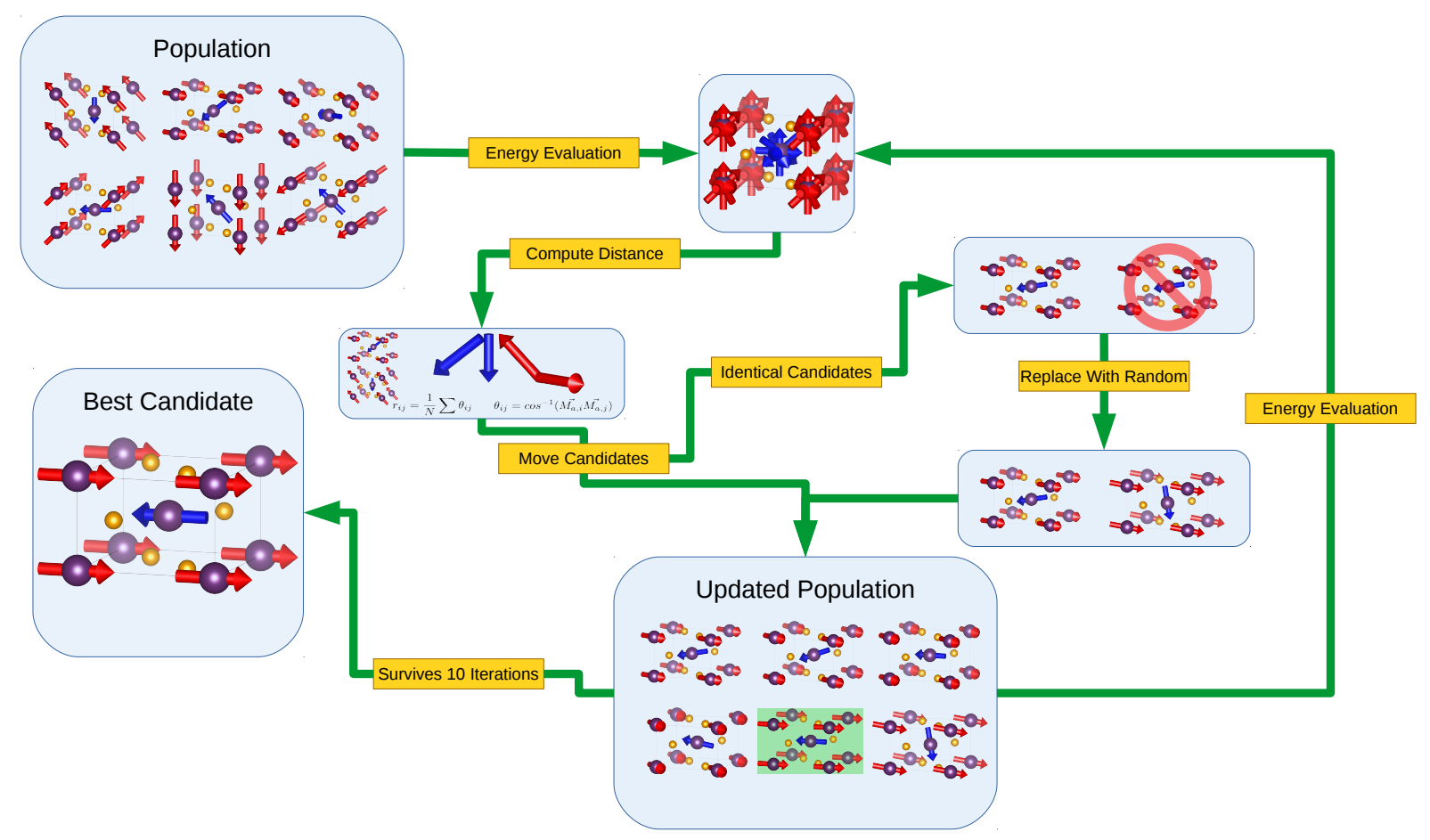

Figure 2.4: Firefly algorithm flowchart. Step one corresponds to the evaluation of the candidates energy. After evaluation, the similarity between pairs of candidates is evaluated by using the distance function. If a candidate is identical to any other candidate, it is discarded. This corresponds to steps 3 and 4 . Step 5 corresponds to when the stabilization limit is reached. This candidate is considered the ground state. 


\section{Chapter 3}

\section{Magnetic Moment Search}

The first application, \outside of structural search, for FA was to the problem of magnetic moment optimization. The magnetic properties of materials are a particularly enticing application for global search methods, from both technological and physical standpoint. Technologically, novel magnetic states could prove to be useful for next-generation storage devices. From the standpoint of basic physics, noncollinear magnetic states are of particular interest since they arise from electron correlation effects, spin-orbit coupling (SOC), surface broken symmetries and/or geometric frustration in strongly correlated materials (SCM)[25]. DFT must be extended to one of the approximations described in the theory chapter with noncollinear magnetic systems, as magnetism ultimately has origins in the exchange-correlation functional problem (as magnetic states in transition metals have their origins in localized d-electrons). For this reason, there has only been limited success in treating non-collinear magnetic systems. This is due to the large number of metastable states that common self-consistent algorithms, such as DFT+U with spin-orbit coupling, fail to handle when the ground state has to be identified [13, 1]. For any single DFT calculation, the initially defined magnetic moment orientation corresponds to a random point on the potential energy surface (PES), which upon relaxation could end in any of these minima, be it a fictitious minima from the approximation to the electron correlation, such as in $\mathrm{LDA}+\mathrm{U}$, or a minima which has a physical meaning. Regardless of origin, most of these minima are very close in energy when compared to the ground state. It was found that the energies between different magnetic moment configurations can be as small as $10^{-5} \mathrm{eV}$. Therefore, search methods which can both identify and differentiate

Adapted with permission from J. Chem. Theory Comput. 2018, 14, 8, 4455-4466. Copyright 2019 American Chemical Society 
between many different minima are necessary to identify the correct ground state. These three test cases will be used to show that FA is well-suited to this problem.

Metaheuristic algorithms can provide a solution to this multiple minima problem. In general, they provide rules for both modifications to different states in the configuration space as well as evaluating how optimal a state is. In other words, metaheuristic methods are used to determine how energetically favorable a given state is. In addition, since metaheuristic methods do not depend on any system specific details, a given algorithm can be applied across a multitude of systems. Here FA is used as it is ideally-suited to the problem at hand. It should be pointed out that though $\mathrm{DFT}+\mathrm{U}$ is the chosen approximation method, the methodology does not rely on this approximation and can be used with any exchange correlation functional or even different methodologies used to describe the system's magnetic properties, as FA merely provides rules for how to generate more energetically favorable candidates; the method does not depend on how the energy of a candidate is evaluated. It must be also be emphasized that the problem of the selection of the value of $U$ and $J$ is not being solved, or any other problems inherent to DFT $+\mathrm{U}$ in the context of ground state prediction. Rather it will be shown that the FA allows for the identification of the energy spectra of all local minima of a magnetic crystal and thus predict which one has the lowest energy. $\mathrm{DFT}+\mathrm{U}$ is used because resource-wise it is comparable to ordinary DFT, and the fictitious minima the method generates provides many different local minima to search over.

Since Equation 2.47 defines FA,appropriate values of the free parameters must be chosen in this expression. From previous results where FA was applied to structural search for phosphorus and Leonard-Jones clusters, as well as several tests cases with small numbers of candidates and high energy tolerances, $\beta=0.8$ and $\gamma=0.3$ where found to yield good results, meaning the strength of attraction between candidates was large enough to modify their magnetic moment values so that the PES was actually explored, and the weighting based of of $\gamma$ was large enough so that little attraction was felt between candidates not in the same basin of attraction, but small enough to capture candidates within the same basin of attraction. These test cases involved at most five candidates, with an energy tolerance of $1 e^{-3} \mathrm{eV}$.

Now that the parameters have been selected, how the algorithm is applied specifically to optimizing magnetic moment configurations can be described in greater detail. Before any calculations proceed, three more parameters must be defined: The generation size, the stabilization limit, and the magnetic atoms. The generation size is the number of candidate configurations per generation. The 
value of this parameter must be considered carefully; too small of a size will cause the searcher to inadequately sample the PES. Too large, and computation time increases without additional accuracy. Thus, this parameter must be chosen in a way that strikes a balance between these two extremes. Based on initial testing and previous work, a generation size of 16 candidates per generation was found to adequately explore the PES. This is also useful considered the architecture of the HPC resources used for the search, as a typical calculation will use 16 nodes. The stabilization limit is the number of generations a lowest-energy candidate must survive to be considered the energy minimum found by the searcher. Caution must be taken with this previous statement, as there are no guarantees the global minimum can ever be found for multi-dimensional surfaces. Similarly to the generation size, a value which is too small will stop the searcher too soon, thus the PES will not be adequately explored, and too large of a size increases computation time with no gain in accuracy. A stabilization limit of 10 generations was found to strike the balance between these two extremes. Finally, the magnetic atoms must be defined. With the implementation of FA in the PyChemia software package, these can be input by hand, or read from the pseudo-potentials which must be supplied for the DFT calculations.

Once these parameters are defined, the searcher can be launched. First, random configurations equal in number to the generation size must be defined. The magnetic moment orientations are found by generating a random unit vector for each magnetic atom. The magnitude of the magnetic moments is determined by stand-alone relaxations before FA is launched. Since both test cases are for previously studied systems, the magnetic moments are initially set to their experimentally known values, and the system is relaxed. This numbers are pulled from a uniform distribution defined on the interval $[0,1)$. Next, the energy of each configuration must be evaluated. This energy can be evaluated with any preferred DFT software package that allows for constraining the magnetic moment directions; the magnetic moment directions must remain fixed during energy evaluation. For all calculations performed, VASP was used to determine the energies of each candidate configuration. After each candidate's energy is evaluated, the distance between each pair of candidates is evaluated. Since physical distance is meaningless when comparing orientations in space, a distance function must be devised in order to evaluate the similarity between candidate configurations. The average of the angle between magnetic moments defined in Equation 3.1, which is the angle between magnetic moments on the same magnetic site, is used for this 
purpose.

$$
\begin{gathered}
r_{i j}=\frac{1}{N} \sum_{k}^{N} \theta_{i j}^{k} \\
\theta_{i j}^{k}=\cos ^{-1}\left(\overrightarrow{M_{k, i}} \overrightarrow{M_{k, j}}\right)
\end{gathered}
$$

Once the distances are evaluated, identical candidates are discarded and replaced with random candidates. Identical here means that the distance between two candidates is a less than a specified distance tolerance. Once this replacement occurs, the population consists of new candidates with random magnetic moments that replaced identical candidates in addition to the moved candidates. This defines the subsequent generations population. Now the algorithm can begin anew with energy evaluation. This process continues iteratively, until the lowest-energy candidate survives for a number of generations equal to the stabalizaiton limit. While this candidate is not guaranteed to be the ground state magnetic configuration, if the stabilization limit is large enough (and the parameters in Eq. 2.47 are defined appropriately), then it should either correspond to or have properties which are close to the true ground state. This problem will be discussed in greater detail when specific results for the searcher applied to test material systems are discussed. This leads into the subsequent sections: Now the application of the algorithm to three test systems; $\mathrm{NiF}_{2}$, a weak ferromagnet with a small canting angle between magnetic moments, $\mathrm{Mn}_{3} \mathrm{Pt}$, and an organic molecule, both frustrated magnetic systems, will be expounded upon.

\section{1 $\quad \mathbf{N i F}_{2}$}

The first test system that FA for magnetic moment optimization was applied to one of the most well-studied weak ferromagnets, $\mathrm{NiF}_{2} . \mathrm{NiF}_{2}$ is a tetragonal crystal with space group $P 4_{2} / \mathrm{m}$. There are six atoms in the unit cell; two Ni atoms and four $\mathrm{F}$ atoms. Only the Ni sites are magnetic. From both experimental and theoretical work, it has been determined that The magnetic moments deviate slightly from an anti-parallel alignment; this deviation manifests as a small canting along the c-axis that drives weak ferromagnetism in this direction [23, 50]. The structural information is listed in Table 3.2. The structural parameters were found from experiment, then convergence test were performed to optimize the energy cut-off as well as the k-point mesh. From these convergence tests, and energy cut-off of $800 \mathrm{eV}$ and a k-point mesh of $6 \times 6 \times 10$ were found to reduce the error in the total 
energy to less than $1^{-8} \mathrm{eV}$. This stringent tolerance is required, as it will be shown that the energy differences between differing magnetic moment configurations can be as low as $1^{-6} \mathrm{eV}$.

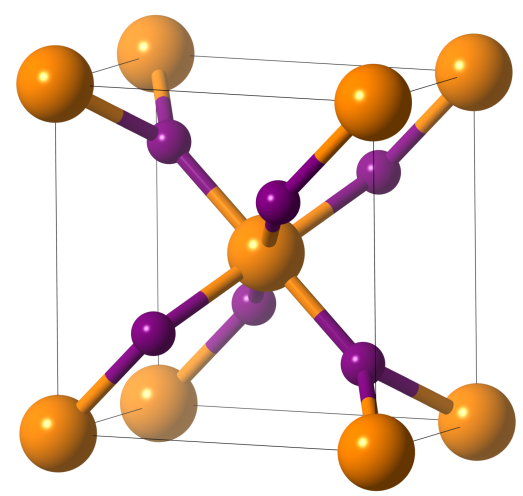

Figure 3.1: Unit cell of $\mathrm{NiF}_{2}$ with space group $P 4_{2} / m$.

\begin{tabular}{|c|c|c|}
\multicolumn{3}{|c}{$\mathbf{N i F}_{2} \boldsymbol{P} 4_{2} / \boldsymbol{m}$} \\
\hline $\mathbf{a}(\AA)$ & $\mathbf{b}(\AA)$ & $\mathbf{c}(\AA)$ \\
\hline 4.54 & 4.54 & 2.99 \\
\hline
\end{tabular}

\begin{tabular}{|c|c|c|c|c|}
\hline Atom & $\begin{array}{c}\text { Wyckoff } \\
\text { Position }\end{array}$ & $\mathrm{x}$ & $\mathrm{y}$ & $\mathrm{z}$ \\
\hline $\mathrm{Ni1}$ & $\mathrm{a}$ & 0.00 & 0.00 & 0.00 \\
$\mathrm{Ni} 2$ & $\mathrm{a}$ & 0.50 & 0.50 & 0.50 \\
F1 & $\mathrm{f}$ & 0.30 & 0.30 & 0.00 \\
F2 & $\mathrm{f}$ & 0.69 & 0.69 & 0.00 \\
F3 & $\mathrm{f}$ & 0.80 & 0.20 & 0.50 \\
F4 & $\mathrm{f}$ & 0.20 & 0.80 & 0.50 \\
\hline
\end{tabular}

Figure 3.2: Structural information for $\mathrm{NiTi}_{2}$ with space group $\mathrm{I} / \mathrm{mmm}$

The canting between different magnetic sites is very small and has been measured to be $0.5^{\circ}[23], 0.38^{\circ}$, [87] and $2.5^{\circ}[75]$. The canting is believed to arise from the magneto-crystalline anisotropy (MCA)[93]. Since LDA+U is used for all calculations, the results should be compared with the experiments, as energies cannot be compared between differing values of $U$ and J. While the MCA energy has never been measured experimentally, theoretically it has been found to vary between 200-300 $\mu \mathrm{eV}$ depending on values of $\mathrm{U}$ and $\mathrm{J}$ which are used.[13] Additionally, it has a bandgap of $6.5 \mathrm{eV}[105]$ and an atomic magnetic moment of $2.213 \pm 0.012 \mu_{B}[95]$. The challenge is to show that FA can locate the multitude of minima which lie in the PES, and to compare them with experimental results. The firefly searcher was ran with sixteen candidates per generation, with a stabilization limit of ten generations. This search was performed values of $\mathrm{U}=1.0,2.0,3.0,4.0,5.0,6.0 \mathrm{eV}$ and $\mathrm{J}=0.0,1.0 \mathrm{eV}$ with $\mathrm{U}>\mathrm{J}$. The results in terms of canting angles for this search are listed in Table 3.1 .

Before any physics is discussed, it must be stressed that the FA works as intended. First it must be shown that the searcher actually explores a significant region of the PES. Secondly, it must be shown that this exploration is both targeted and is fundamentally different than that of a random search. Finally, as the 
generation increases, it must be shown that the energy of the low-energy candidate decreases until a lowest-energy candidate is found.

Figure 3.4 shows the initial canting angles and angles with respect to the $\mathrm{x}$-axis and of each candidate for $\mathrm{U}=2 \mathrm{eV}, \mathrm{J}=0 \mathrm{eV}$. It is clear that the searcher explored a large region of the PES. Figure 3.5 displays the targeted nature of FA more convincingly. Each node represents a single candidate, that is, each node corresponds to a unique point on the PES. For this case, there were 563 unique candidates evaluated. It must be noted that the spatial extent of this plot has no physical meaning, only whether or not candidates are connected is relevant. The blue regions correspond to the lowest energy candidates evaluated. If two nodes are linked, that means that their magnetic moment orientations are similar to one another. If a node is not linked, this means that the magnetic moment configuration which corresponds to the node is not similar to any other candidate configuration. While the searcher does explore a large region, i.e. there are many disconnected points, the density of points near the minima is significantly greater. The degree of connectedness of a plot can be numerically evaluated. Here the average connectivity is used, which is the average of the maximum number of disjoint paths between nodes [10]. For this plot, the average connectivity is 0.229 . This is because FA prefers to probe the PES near minima. From this figure, it can be seen that there are three regions which have been extensively explored. Figure 3.10 displays the different minima located for $\mathrm{NiF}_{2}$, with $\mathrm{U}=4 \mathrm{eV} \mathrm{J}=0 \mathrm{eV}$. It can be seen that a multitude of minima where located with the use of sixteen candidates per generation. In the zoomed inset, there are multiple regions of clumped candidates; these are the regions around different minima which the searcher explores. This provides futher evidence that the PES was adequately explored.

Figure 3.6 shows the semi-log of the energy difference between the lowestenergy configuration in each generation and the overall best candidate for $J=0$ and $\mathrm{J}=1$. Several things are apparent from the plot. First, the energy of the bestcandidate decreases in each subsequent generation. This shows two things: As the searcher evolves, better candidate magnetic configurations are found. Additionally, this means that the searcher is sampling different points in configuration space. Secondly, the energy differences between candidates is small, as the maximum range between the starting best candidate and final best candidate is only on the order of $10^{-1} \mathrm{eV}$. This is unsurprising, as the energy difference between configurations corresponding to different local minima is only in the order of $1 \mathrm{meV}$. In Figure 3.6, it can be seen that the energy difference is less than $1 \mathrm{meV}$ after 15 generations. This means that the firefly searcher has located a magnetic moment configuration with magnetic moments which are perturbed slightly from its mini- 


\begin{tabular}{ccccccc}
\hline $\mathrm{U}(\mathrm{eV})$ & $\mathrm{J}(\mathrm{eV})$ & Canting Angle $\left(^{\circ}\right)$ & & $\mathrm{U}(\mathrm{eV})$ & $\mathrm{J}(\mathrm{eV})$ & Canting Angle $\left(^{\circ}\right)$ \\
\cline { 1 - 3 } \cline { 5 - 6 } 1.0 & 0.0 & 0.000 & & 3.5 & 0.0 & 0.073 \\
1.0 & 0.0 & 0.036 & & 4.0 & 0.0 & 0.088 \\
1.5 & 0.0 & 0.050 & & 4.0 & 1.0 & 0.247 \\
1.5 & 1.0 & 0.078 & & 4.5 & 0.0 & 0.109 \\
2.0 & 0.0 & 0.031 & & 5.0 & 0.0 & 0.122 \\
2.0 & 1.0 & 0.122 & & 5.0 & 1.0 & 0.327 \\
2.5 & 0.0 & 0.069 & & 5.5 & 0.0 & 0.205 \\
3.0 & 0.0 & 0.067 & & 6.0 & 1.0 & 0.425 \\
3.0 & 1.0 & 0.048 & & &
\end{tabular}

Table 3.1: Canting angle and energies of best candidates for all values of $U$ and $J$ considered for $\mathrm{NiF}_{2}$ after relaxation.

mum value. Since the FA efficiently searches in the local area around a candidate configuration in configuration space, it is unsurprising that once the searcher locates a candidate which is close to a minima, it only takes a few generations to find that minima.

After the searcher has completed, each candidate should be relaxed with the constraint removed to check if the best candidate truly lies in a minima on the PES, as well as to see how many minima lie close to the best candidate. This is done because it is possible that FA finds low-energy candidates which lie in different basins of attraction, it may not actually reach the true minima. However since the goal of this method is to show that it explores the many minima on the PES, showing that the candidates that the FA generates lie in different basins is enough to show that it explores different regions of the PES. Figure 3.3 shows the energy after relaxation of the last generation for $\mathrm{U}=2 \mathrm{eV} \mathrm{J}=0.6 \mathrm{eV}$.

To show that this minima corresponds to the ground state, the canting angles of the best candidate found will be compared to the canting angles reported experimentally for $\mathrm{NiF}_{2}$. The results are also compared to the experimentally measured bandgap and magnetic moment magnitude. Figure 3.7 shows the canting angles as a function of $U$ for $J=0$ and $J=1$, before relaxation. It can seen that there is no obvious trend between the value of $U$ and the canting angle. Figure 3.8 shows this relationship after relaxation. This further highlights the importance of relaxation as there is an overall trend towards an increase in the canting angle as the value of $\mathrm{U}$ increases. The discrepancies in this plot are due to the candidates which do not relax into the true ground state candidate. 


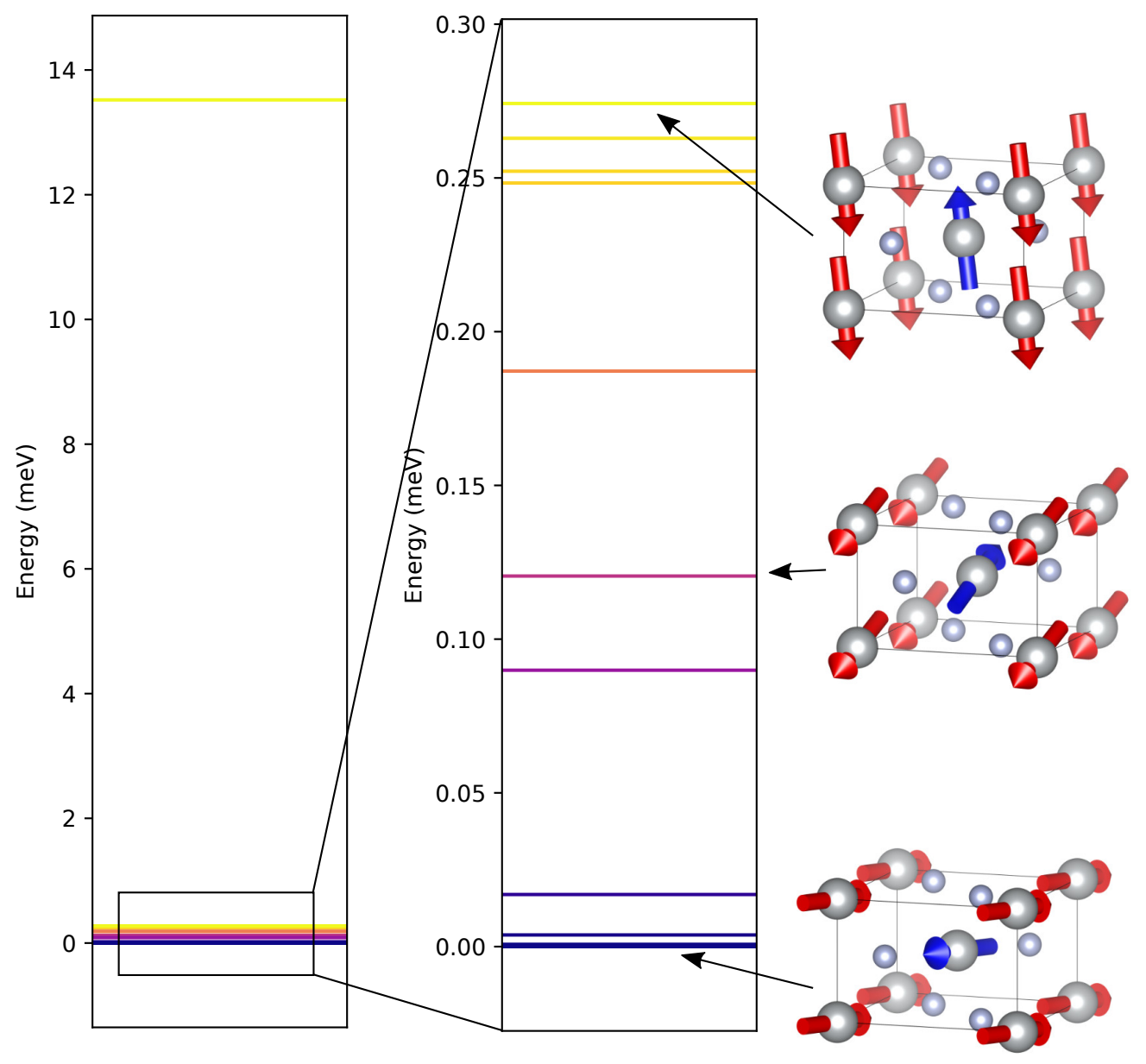

Figure 3.3: Energies of the last generation of candidates after relaxation for $U=2 \mathrm{eV}$ $\mathrm{J}=0.6 \mathrm{eV}$. The left energy plot of the figure displays all of the candidates in the final generation. The middle plot is the zoomed in portion of the plot on the left. From this plot, there are three minima that are within $1^{-4} \mathrm{eV}$ of the lowest energy-candidate. 
In Figure 3.9 it is shown that the energy difference between the lowest energy candidate found for a given $U$ for $J=1$ and all other candidates of the same $U$ as a function of the canting angle. The dependence between the canting angle and $\mathrm{U}$ is expected to be monotonic, yet the results observed in Figure 3.7 show a dependence which is not monotonic. This is because two candidates with the same canting angle can have magnetic moments that are orientated in different directions with respect to the c-axis, which means they will have different energies after evaluation. This highlights the importance of removing the magnetic constraint, as candidates with magnetic moments that are not parallel to the c-axis will adjust their magnetic moments to lie in this plane. This is why there is such a drastic change between Figures 3.7 and 3.8 .

Experimentally, the magnetic moments lie in a plane perpendicular to the caxis. [50, 23], and the majority of the lowest-energy candidates after relaxation are in agreement with this. Each low-energy candidate has both bandgap and magnetic moments that are lower than experimentally measured values. The maximal value found for the magnetic moment is for $\mathrm{U}=6 \mathrm{eV}, \mathrm{J}=1 \mathrm{eV}$, and it is $1.76 \mu_{B}$. The maximal bandgap found was also for $\mathrm{U}=6 \mathrm{eV}, \mathrm{J}=1 \mathrm{eV}$, and it is $4.82 \mathrm{eV}$. Experimentally, these values are slightly higher than the values found, as the magnetic moment is measured to be $2.23 \mu_{B}$, and the bandgap is $6.5 \mathrm{eV}$.

While FA has been able to locate the lowest energy configuration for a system with single global minima, i.e. in $\mathrm{NiF}_{2}$, it is important to test that it is also able to find solutions which correspond to degenerate states with several and different magnetic configuration, e.g. in $\mathrm{Mn}_{3} \mathrm{Pt}$. 


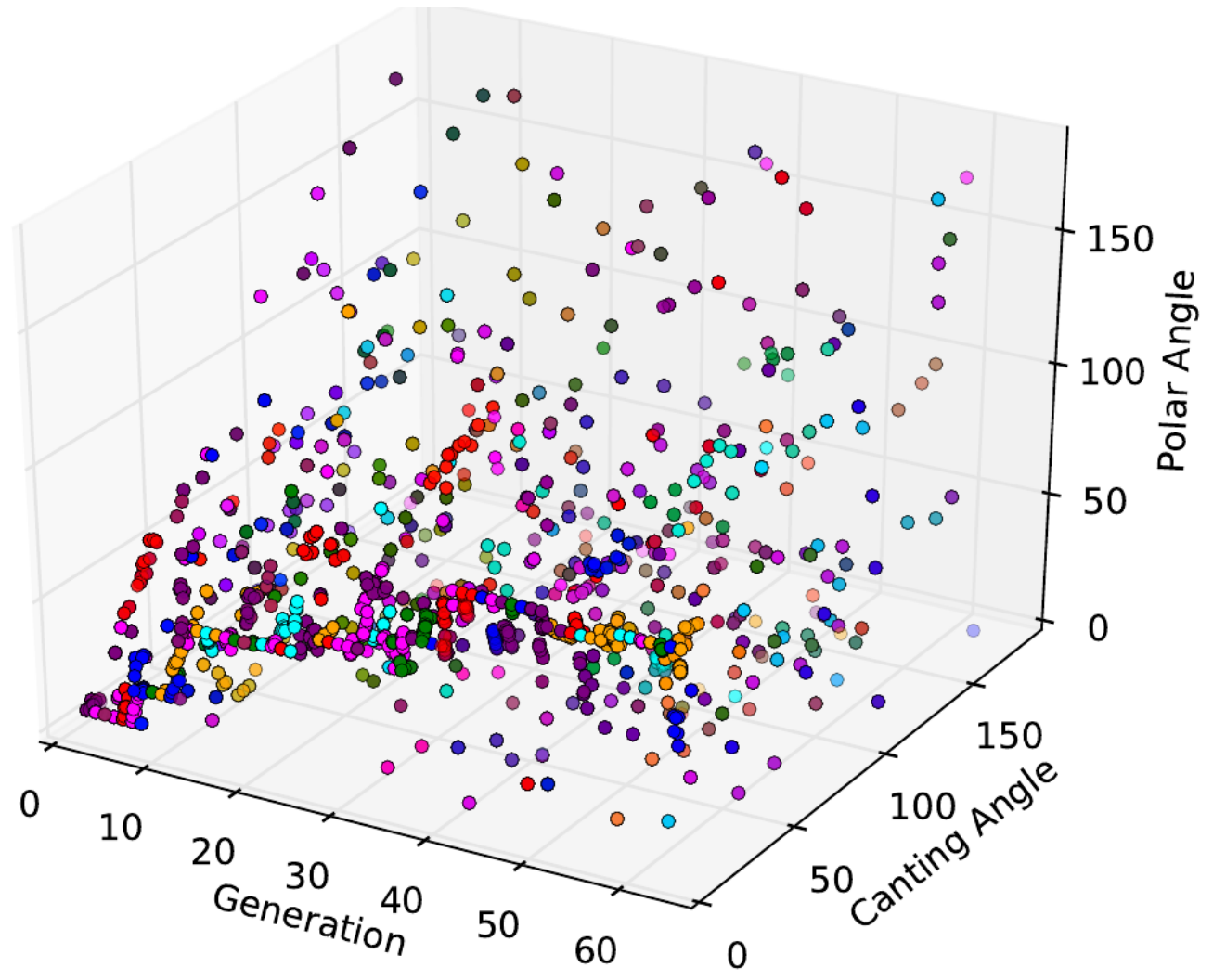

Figure 3.4: Initial magnetic moment configurations of candidates evaluated for $U=2 \mathrm{eV}$, $\mathrm{J}=0.6 \mathrm{eV}$. 


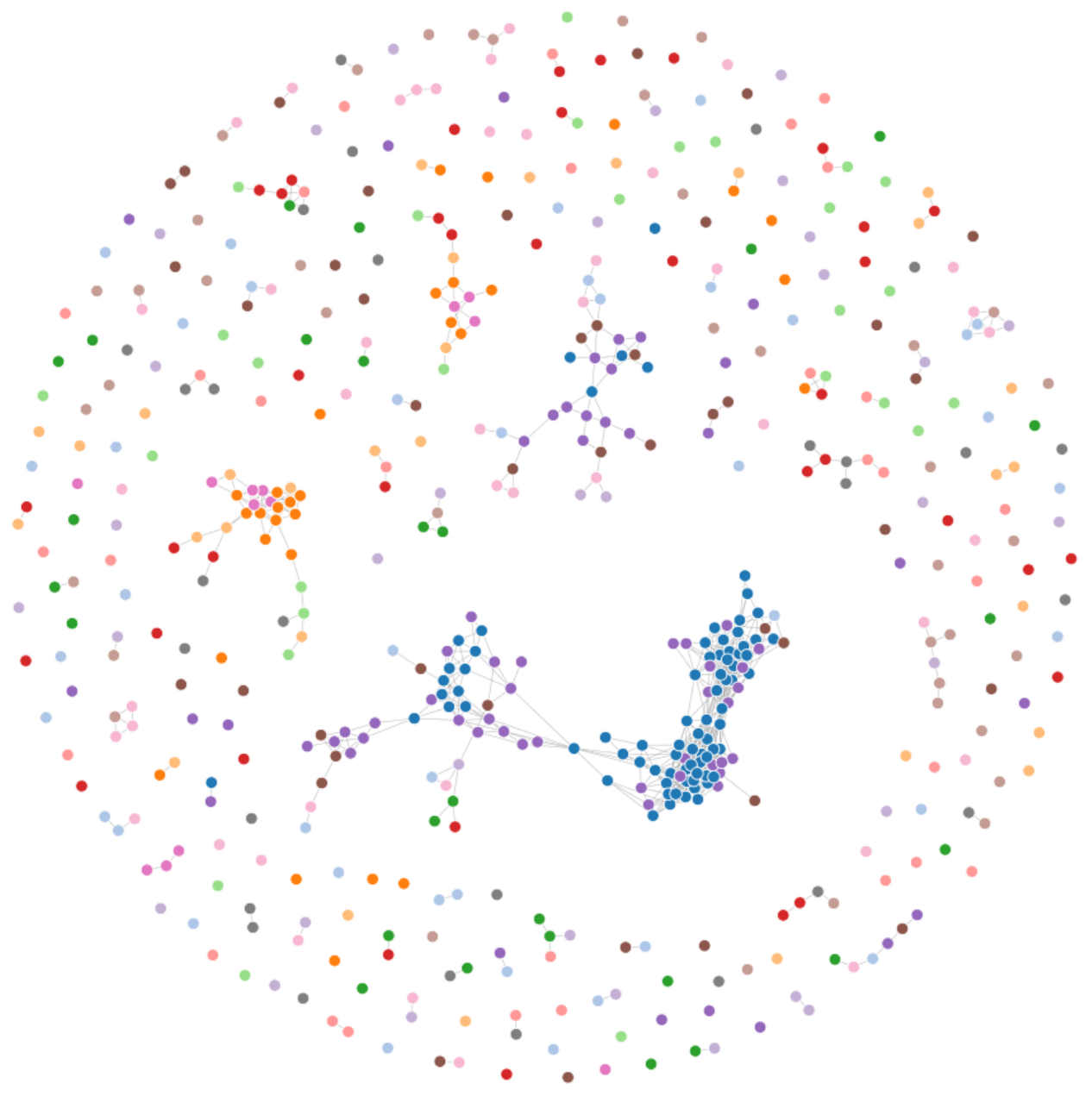

Figure 3.5: Network plot of the entire search for $\mathrm{U}=2 \mathrm{eV}, \mathrm{J}=0 \mathrm{eV}$. The colors denote the energy, where blue is the lowest energy and red is the highest. If two nodes are linked, the link represents Equations (3.1) and 3.2) where $r_{i j}<0.3$ Many of the points are disconnected from the entire plot, this means these candidates where not close to any others after initial evaluation. These points represent the stoichiastic aspect of FA, in that they are the randomly generated initial candidates as well as the replacements when duplicates are found. The other three regions which are highly linked show us the targeted nature of FA, that is that it extensively probes the regions around low energy candidates. 

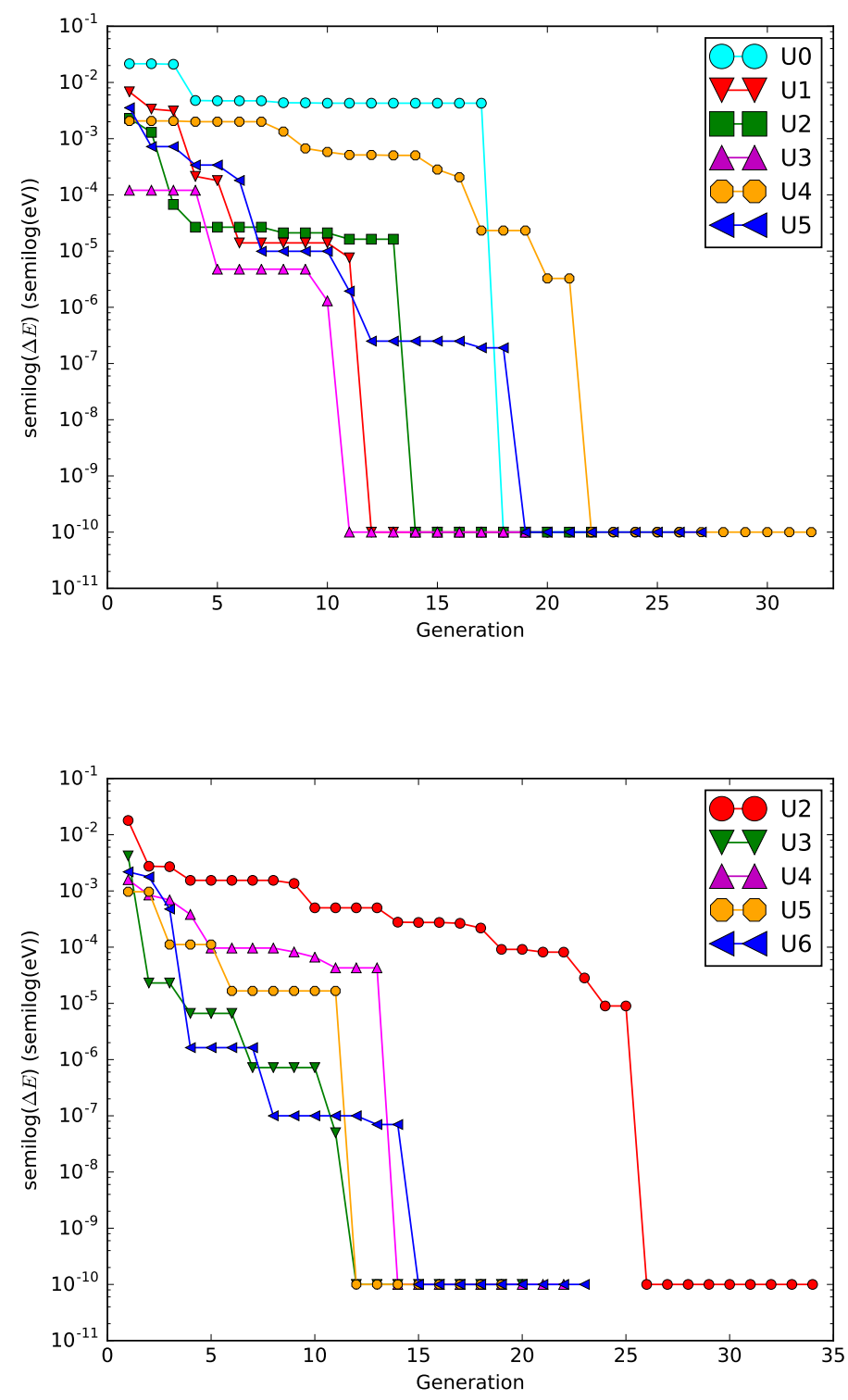

Figure 3.6: Energy difference between the final best candidate and the best candidate of each generation for both $\mathrm{J}=0$ and $\mathrm{J}=1 \mathrm{eV}$. This plot is logarithmic in order to show multiple values of $U$ on the same plot. The ten points corresponding to the ten generations in which a structure must survive to be deemed the final best candidate are where each case plateaus. While the energy difference should be $0 \mathrm{eV}$ in this case, the difference is plotted at a value which is less than the energy tolerance of every calculation. The left plot is for $\mathrm{J}=0 \mathrm{eV}$, the right plot is $\mathrm{J}=1 \mathrm{eV}$. 


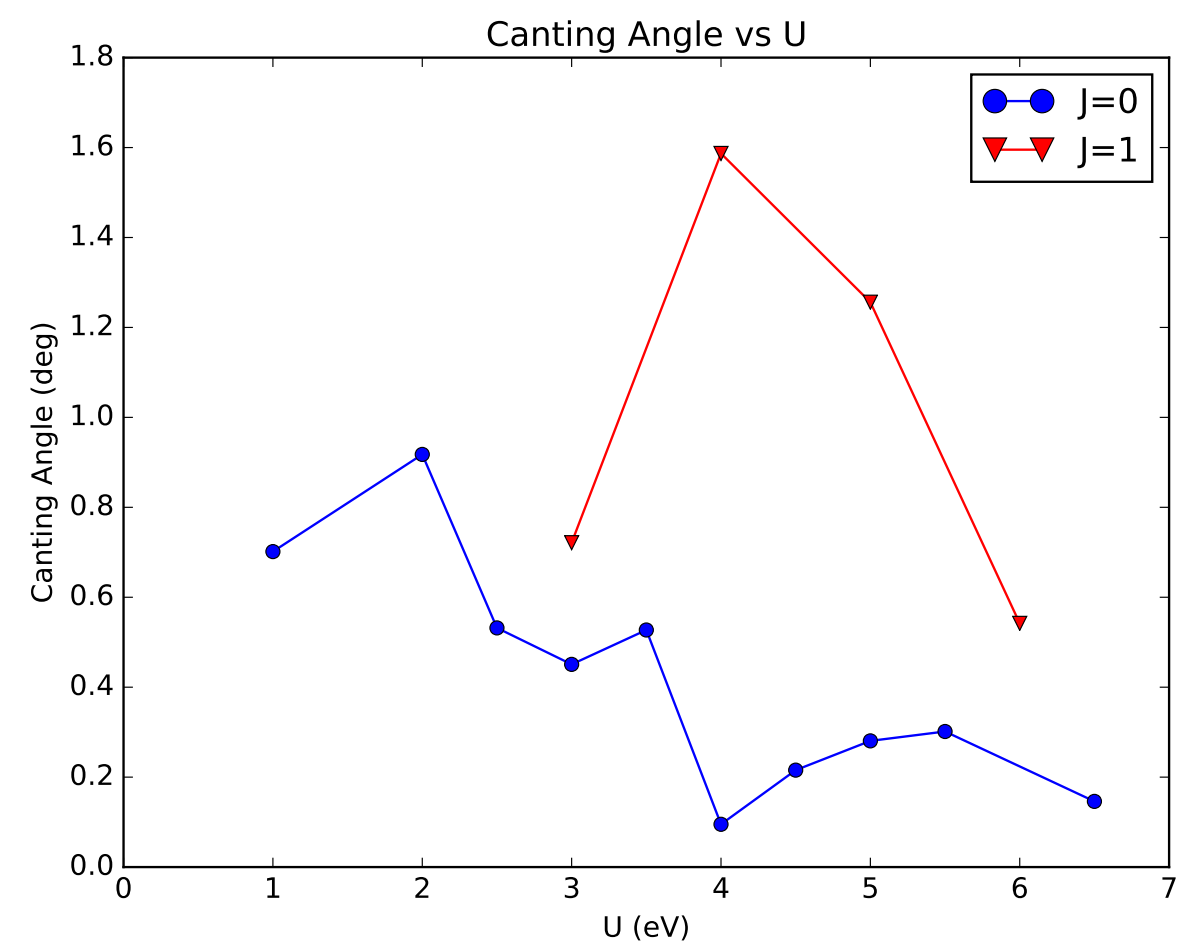

Figure 3.7: Canting angle for all values of $U$ and $J$ which have been considered, before relaxation.

\section{2 $\mathrm{MnPt}_{1}$}

THe second application of the FA algorithm is with $\mathrm{Mn}_{3} \mathrm{Pt}$. This system exhibit a first order magnetic transition between two different magnetic phases at $400 \mathrm{~K}$ [59]. The low temperature phase is of interest since it is known to display frustration.[59] Neutron diffraction experiments have shown that the magnetic moments of the Mn atoms in this phase are along the [111] direction of the unit cell.[61] Additionally, the average magnetic moment of the Mn atoms is $3.0 \pm 0.3 \mu_{B}$. [65]. In the exact same fashion as $\mathrm{NiF}_{2}$, the structure was initially defined in term of experimental results, then the energy cutoff and the k-point mesh were converged to the same tolerance as with $\mathrm{NiF}_{2}$. Here, and energy cutoff of $800 \mathrm{eV}$ and a k-point mesh of 16x16x16 were enough to reach this tolerance. Finally, geomet- 


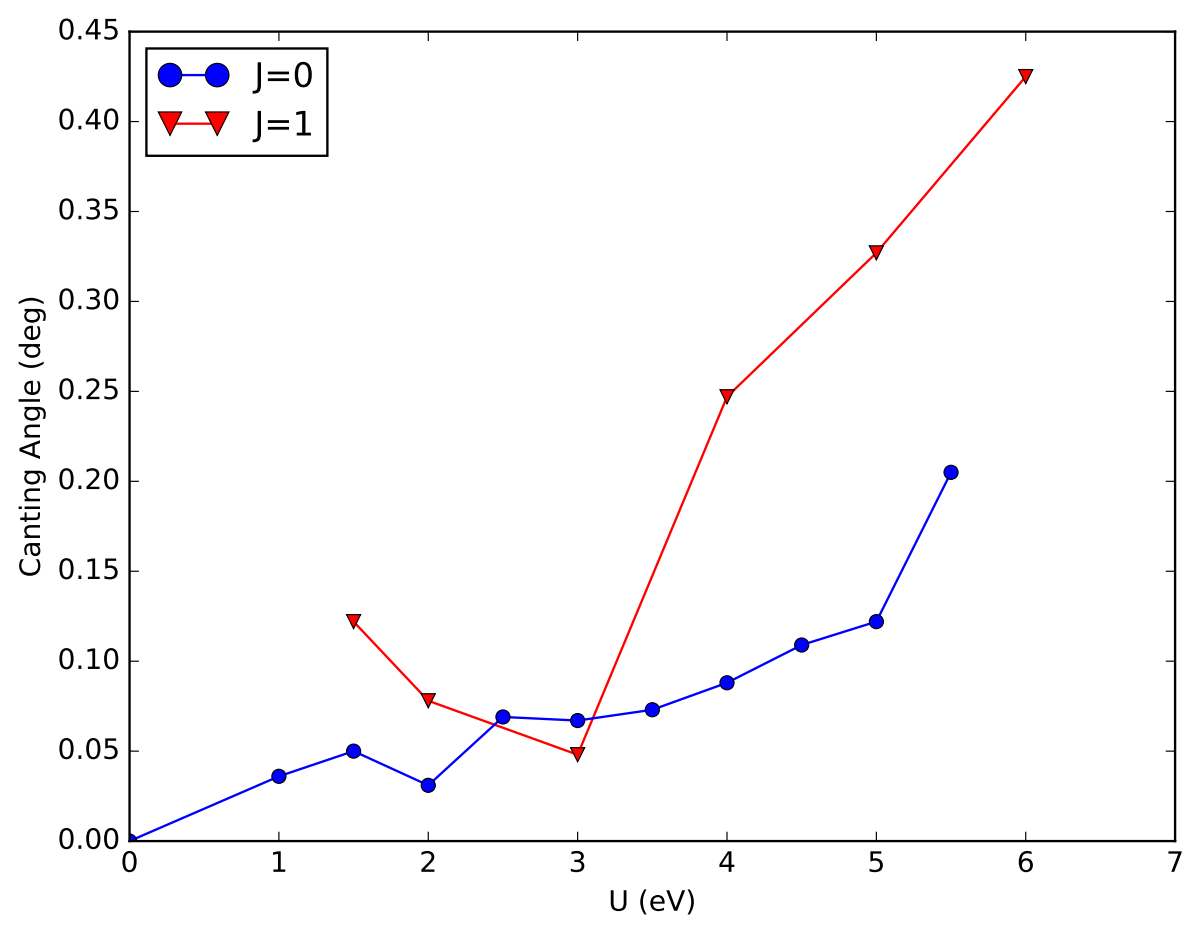

Figure 3.8: Canting angle for all values of $U$ and $J$ which have been considered after removing the constraint on the magnetic moment direction. This ensures that each candidate relaxes to a minima on the PES.

ric relaxations were performed in order to full converge the cell geometry. The structural information from this process is displayed in Table 3.12.

Similarly to the previous example, first it is shown that the FA performs as intended. Figure 3.14 shows each different initial magnetic moment configurations evaluated for $\mathrm{U}=4 \mathrm{eV}, \mathrm{J}=0 \mathrm{eV}$. The searcher evaluated a significant portion of configuration space. Also there are several regions where the evaluated points are very close to one another. As for $\mathrm{NiF}_{2}$, a network plot shows the targeted nature of the FA more convincingly. Figure 3.13 displays the network plot for $\mathrm{U}=2.0$, $\mathrm{J}=0.0$. Similarly to the plot for $\mathrm{NiF}_{2}$ each node corresponds to one candidate, and two nodes are linked only if their distance function evaluates to a value which is less than 0.3. The number of nodes in the plot is 496, which means that 496 unique candidates were evaluated. Just as before, the average connectivity can 


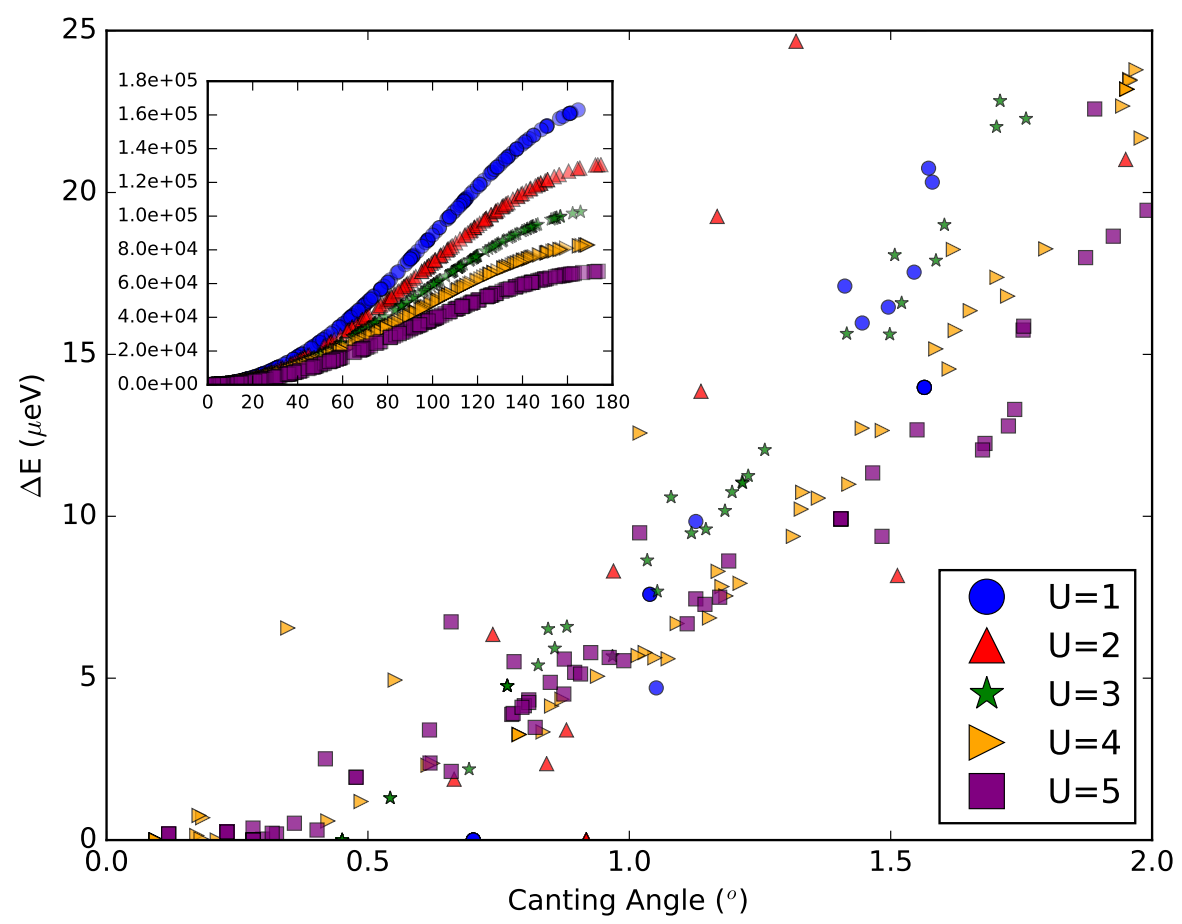

Figure 3.9: Energy difference for all candidates as a function of their canting angles for $\mathrm{J}=1 \mathrm{eV}$.

be found, and it is 0.125 , which means it is less connected than the plot for $\mathrm{NiF}_{2}$. Considering that $\mathrm{Mn}_{3} \mathrm{Pt}$ is a frustrated system and $\mathrm{NiF}_{2}$ is not, the graph should be more disconnected, as there should be several disconnected islands of connected nodes, with each island corresponding to a frustrated state.

Figure 3.15 shows the semilog plot of the energy difference between the best candidate and the lowest energy candidate for each generation. As expected, the energy difference decreases as the number of generations increase as the FA is locating better and better candidates as the search evolves. It is also clear that as the $U$ value increases the potential energy surface becomes more rough and the location of the lowest energy configuration needs more iterations. This is consistent with the fact that as $U$ increases, the electron-electron correlation increases, which makes magnetic energy barriers larger and the exploration of the surface becomes more difficult, though FA is still able to find the right minima even at 


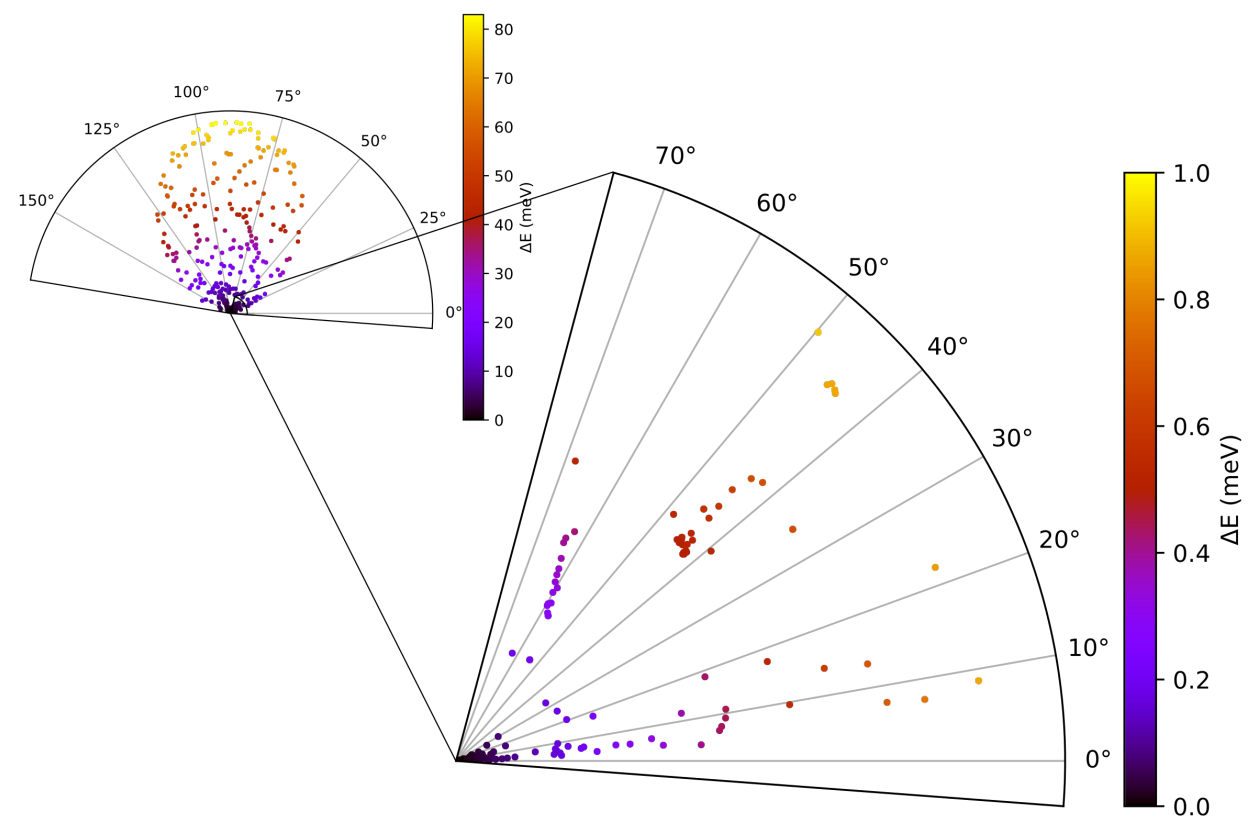

Figure 3.10: Polar plot of the distances and energies of all candidates relative to the lowest energy candidate for $\mathrm{NiF}_{2}$, with $\mathrm{U}=4 \mathrm{eV} \mathrm{J}=0 \mathrm{eV}$. The energy is relative to the lowest energy candidate found. The smaller plot is the plot for every candidate, and the zoomed plot is for candidates whose energy is less than $1 \mathrm{meV}$ higher than the lowest energy candidate. The distance function is defined as the average angle between magnetic sites between two candidates, so slices of constant angle correspond to candidates which are the same distance away from the lowest energy candidate. Candidates form a number of clumps in the zoomed plot, these clumps are the different minima which were located. 


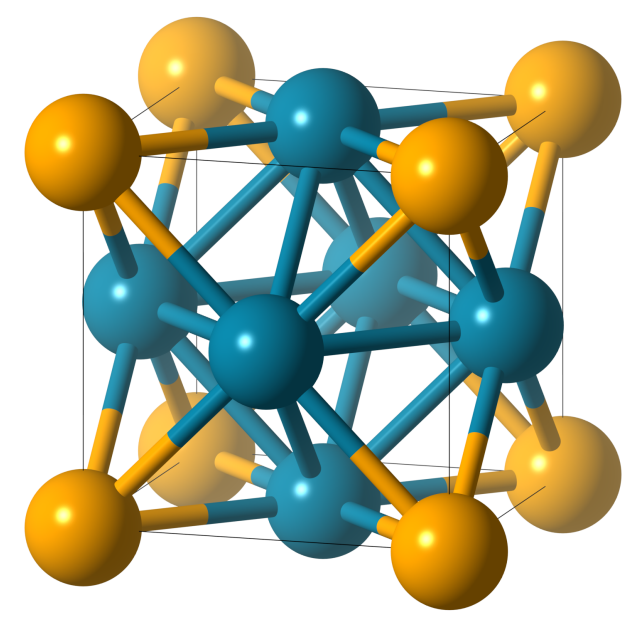

Figure 3.11: Unit cell of $\mathrm{Mn}_{3} \mathrm{Pt}$ with space group $P m-3 m$

\begin{tabular}{|c|c|c|c|c|}
\hline \multicolumn{5}{|c|}{$\mathrm{Mn}_{3} \mathrm{Pt} P \boldsymbol{P m}-3 m$} \\
\hline $\mathbf{a}(\stackrel{\circ}{A})$ & \multicolumn{2}{|c|}{$\mathbf{b}(\stackrel{\circ}{A})$} & \multicolumn{2}{|c|}{$\mathbf{c}(\stackrel{\circ}{A})$} \\
\hline 3.63 & \multicolumn{2}{|c|}{3.63} & \multicolumn{2}{|c|}{3.63} \\
\hline Atom & $\begin{array}{l}\text { Wyckoff } \\
\text { Position }\end{array}$ & $\mathrm{x}$ & $\mathrm{y}$ & $\mathrm{Z}$ \\
\hline Mn1 & $\mathrm{a}$ & 0.00 & 0.50 & 0.50 \\
\hline Mn2 & $\mathrm{a}$ & 0.50 & 0.50 & 0.00 \\
\hline Mn3 & f & 0.50 & 0.00 & 0.50 \\
\hline Pt1 & $\mathrm{f}$ & 0.00 & 0.00 & 0.00 \\
\hline
\end{tabular}

Figure 3.12: Structural information for $\mathrm{Mn}_{3} \mathrm{Pt}$ with space group $\mathrm{Pm}-3 \mathrm{~m}$

large $U$ values.

The search was performed for $\mathrm{U}$ values of $0.0,1.0,2.0,3.0 \mathrm{eV}$, and $4.0 \mathrm{eV}$ for $\mathrm{J}$ $=0 \mathrm{eV}$. Figure 3.16 shows the results for the magnetic moment magnitudes for all low-energy candidates which have been found after relaxation. Two cases were found in which two lowest-energy candidates have magnetic moment magnitudes that agree with experiment, $\mathrm{U}=2 \mathrm{eV}$ and $\mathrm{U}=3 \mathrm{eV}$.

It must also be determined that FA yields magnetic moment orientations that agree with experiment. Figure 3.18 shows the magnetic moments of two candidates after relaxation, $\mathrm{U}=2.0$ and $\mathrm{U}=1.0$. Only $\mathrm{U}=2.0$ has magnetic moments which lie in the [111] plane. Combining these two results together,the low energy candidate found for $\mathrm{U}=2 \mathrm{eV}, \mathrm{J}=0 \mathrm{eV}$ is consistent with experimental measurements. Additionally, previous computational studies have identified three potential configurations which agree with neutron diffraction experiments. [59]

The diversity of the minima of the relaxed generation can also be checked. Since the lowest energy candidate should lie in the [111] plane, the relative orientation of the plane the magnetic moments lie in can be used to differentiate results. This result is displayed Figure 3.17 for $\mathrm{U}=2.0, \mathrm{~J}=0.0$. Unsurprisingly, most of the candidates relax to a configuration which is either in the [111] plane, or is close to the [111] plane. Most of the candidates lie within $0.5 \mathrm{meV}$ above the lowest-energy candidate. The candidate which is $3 \mathrm{meV}$ higher in energy can be identified with another theoretically predicted orientation which agrees with experiment, even though it is not exactly in the [111] plane. [59] This orientation 
was predicted to be $2.8 \mathrm{meV}$ higher in energy than the lowest-energy orientation, a result that is in agreement with the performed calculations. Since $\mathrm{Mn}_{3} \mathrm{Pt}$ is a frustrated system, there should be at least one more magnetic moment configuration which has the same energy as the lowest-energy candidate. The third configuration identified in Figure 3.17 is only $1 \mu \mathrm{eV}$ higher in energy than the lowest-energy candidate. Since this energy difference is significantly lower than what was previously reported, this candidate is identified as a degenerate configuration. Thus, the expected degeneracy in this system has been reproduced by the FA.

\section{$3.3\left(\mathbf{M n}_{I V}\right)_{3} \mathbf{O}_{4} \mathbf{L}_{4}\left(\mathbf{H}_{2} \mathbf{O}\right)$}

The final application of FA for magnetic moment optimization is with the molecular system $\left(\mathrm{Mn}_{I V}\right)_{3} \mathrm{O}_{4} \mathrm{~L}_{4}\left(\mathrm{H}_{2} \mathrm{O}\right)$, where $\mathrm{L}=N, N^{\prime}$-bis(methylene)- $Z$-1,2-ethenediamine. This molecular system mimics the oxomanganese complex of photosystem II, and the action of this complex causes the oxidation of water to dioxygen. Because of this, the considered molecular system is a simplified version of the well-studied $\left(\mathrm{Mn}_{I V}\right)_{3} \mathrm{O}_{4}$ (bpy) $)_{4}\left(\mathrm{H}_{2} \mathrm{O}\right)$ system [90]. This oxidation is a key step in the utilization of solar energy in biological systems. Experimental work with $\left(\mathrm{Mn}_{I V}\right)_{3} \mathrm{O}_{4}(\mathrm{bpy})_{4}\left(\mathrm{H}_{2} \mathrm{O}\right)$ has indicated that strong anti-ferromagnetic coupling exists between the spin centers. The three oxomanganese complexes in this molecular system are aligned such that the Mn atoms in each complex lie on the vertices of a triangle, similarly to the case for $\mathrm{Mn}_{3} \mathrm{Pt}$. Because of this, it is believed that competition between the three spin centers leads to frustration. The theoretical work of Luo et. al. [70] showed that if the spins of these atoms adopt a non-collinear configuration, then this frustration is minimized, resulting in a state lower in energy than the previously expected frustrated collinear configurations. Since this system has been shown previously to display a non-collinear spin arrangement, it provides a molecular system where FA can be tested. Luo et. al. found a ground state configuration where the magnetic moments of the Mn atoms lie in the $\mathrm{x}-\mathrm{y}$ plane, where each magnetic moment points outwards from the center of the triangle connecting the three $\mathrm{Mn}$ atoms. The energy difference between this non-collinear state and the lowest-energy collinear state is $0.3 \mathrm{eV}$. While they also considered excited states of this molecule, this are not necessary for the purposes of the search, the goal is to predict the correct ground state. Values of $\mathrm{U}=0,1$, and $2 \mathrm{eV}$, with $\mathrm{J}=0 \mathrm{eV}$ where selected for the search based on the results for $\mathrm{Mn}_{3} \mathrm{Pt}$, since the $\mathrm{Mn}$ atoms are the magnetic atoms in both systems. As with the previous cases, it must be 
shown that a significant portion of configuration space is explored. Figure 3.19 displays the network plot obtaineded for $\left(\mathrm{Mn}_{I V}\right)_{3} \mathrm{O}_{4} \mathrm{~L}_{4}\left(\mathrm{H}_{2} \mathrm{O}\right)$. As discussed for the previous two cases, each node corresponds to a single candidate, and nodes are linked based on their distance from one another. As before, the distance cutoff was specified to be 0.3 , on a scale where 0.1 specifies candidates which are identical. Since the average angles between magnetic sites are used to define the distance, this cut-off means the average angle must be less than 0.3 radians between magnetic moment sites. Just as in the previous cases, the graph is primarily a collection of completely disconnected nodes and highly connected nodes, which shows that the searcher is targeted. From this plot, a multitude of connected regions which are mutually disconnected can be seen. Just as before, this shows that FA can explore multiple regions of the PES simultaneously. There are 424 nodes, which means there were 424 unique magnetic moment configurations which were evaluated by FA.

Figure 3.20 shows the energy difference between different candidates for the final generation for $\mathrm{U}=2 \mathrm{eV} \mathrm{J}=0 \mathrm{eV}$. The first remark that can be made is that the energy differences between minima is in the order of $0.1 \mathrm{meV}$. The second remark is that 16 different minima were located in this final generation. This, coupled with the network plot in Figure 3.19, show that a calculation that starts with initial conditions that correspond to a random point on the PES has very little hope to relax into the global minima.

Finally, the lowest energy candidate has properties which agree with the results from the work of Luo et. al. The magnetic moments lie in the a-c plane, and they are oriented pointing outward from the center of the triangle where the $\mathrm{Mn}$ atoms lie on. However, the results yield magnetic moment magnitudes which are significantly higher than those previously reported. Figure 3.21 displays the average magnetic moment magnitude of the best candidate for the values of $U$ and $\mathrm{J}$ considered, along with the value from the previous theoretical study. The disagreement between the performed calculations wtih previous theoretical work is not a failure of the FA, however, but is more than likely due to the use of a different exchange-correlation functional as well as the use of LDA+U. Experimentally, the reported magnetic moments of $\left(\mathrm{Mn}_{I V}\right)_{3} \mathrm{O}_{4}(\mathrm{bpy})_{4}\left(\mathrm{H}_{2} \mathrm{O}\right)$ go from $1.71 \mu_{B}$ at 4.6 $\mathrm{K}$ to $3.77 \mu_{B}$ at $292 \mathrm{~K}$. [90], which is higher than those reported by Luo et. al and in better agreement with the low-energy candidate found with FA. 


\subsection{Chapter Summary}

In this chapter, FA was applied to the problem of magnetic moment prediction. Three test cases were chosen to show that FA performs well across a multitude of systems with significantly different properties. These were the weak ferromagnetic $\mathrm{NiF}_{2}$, the frustrated crystall $\mathrm{Mn}_{3} \mathrm{Pt}$, and a molecular system, $\left(\mathrm{Mn}_{I V}\right)_{3} \mathrm{O}_{4}(\mathrm{bpy})_{4}\left(\mathrm{H}_{2} \mathrm{O}\right)$. In each case, FA was able to find low-energy states that were in agreement with previous results, without any reference to system specific details. For $\mathrm{NiF}_{2}, \mathrm{FA}$ was able to generate low-energy states across a range of $U$ and $J$ values that yielded the correct canting angle, without any inital reference to symmetry, the MCA, or any other properties which are specific to $\mathrm{NiF}_{2}$. For $\mathrm{Mn}_{3} \mathrm{Pt}$, not only was FA able to locate the correct ground-state magnetic configuration, it was also able to locate a second previously reported orientation which agrees with experiment. Additionally, the magnetic moments were magnetic moments were found to lie in the [111] plane across several values of $U$ and $J$, which relaxed to the correct orientation. This inidcates that FA is able to find configurations which are very close to the minima, even without relaxing each candidate during the run of the searcher. For $\left(\mathrm{Mn}_{I V}\right)_{3} \mathrm{O}_{4}(\mathrm{bpy})_{4}\left(\mathrm{H}_{2} \mathrm{O}\right)$, FA was able to locate a low-energy magnetic moment configuration which agrees with previous theoretical work. For all three cases, a large number of metastable states were found, which enabled the differentiation between multiple low-energy states so that the true ground state could be found. This improves upon the weaknesses of currently used methods in magnetic systems. 


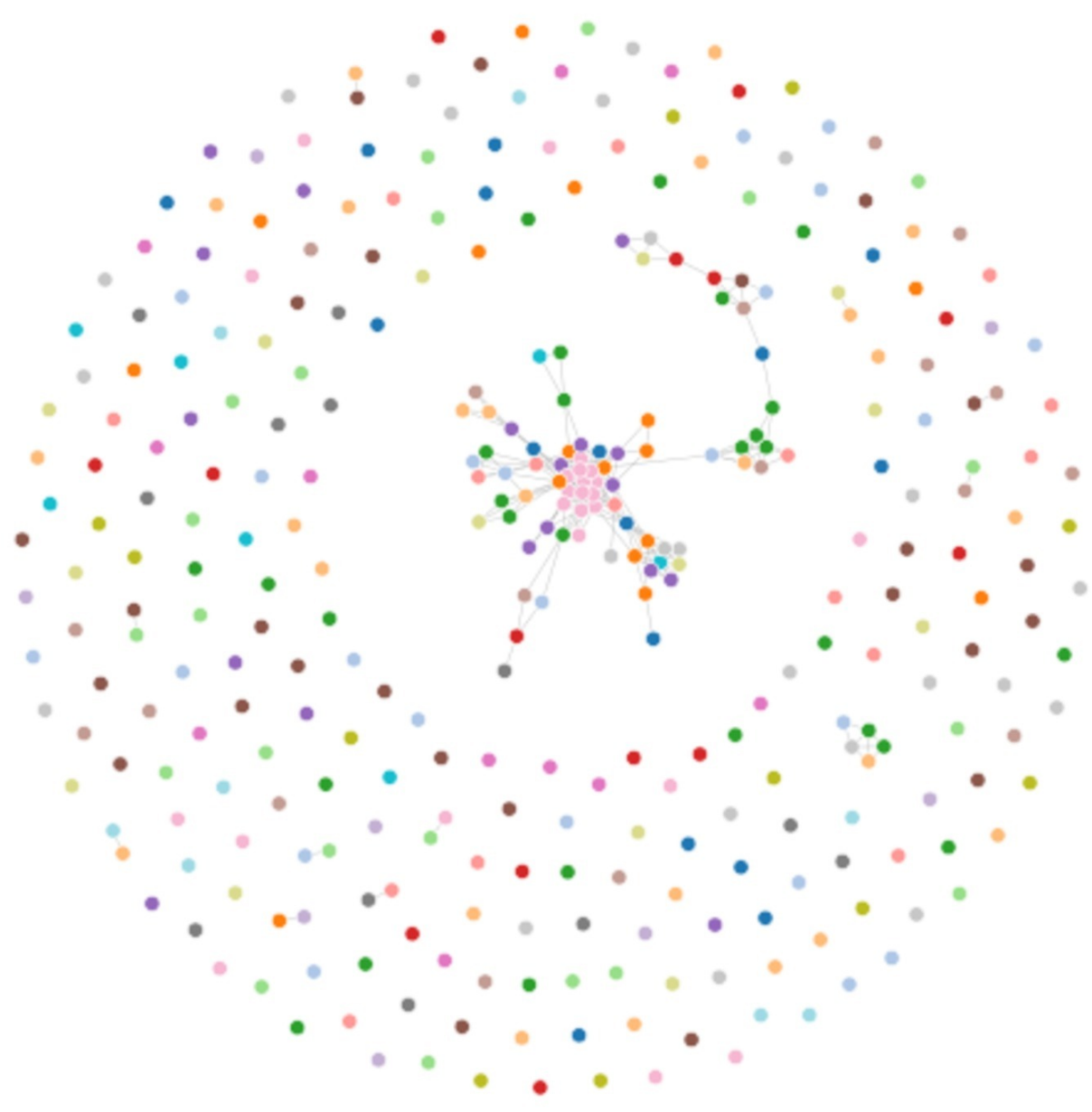

Figure 3.13: Network plot of the entire search for $\mathrm{U}=4 \mathrm{eV}, \mathrm{J}=0 \mathrm{eV}$ in $\mathrm{Mn}_{3}$ Pt. The colors denote the energy, where blue is the lowest energy and red is the highest. If two nodes are linked, the link represents Equations (3.1) and (3.2) where $r_{i j}<0.3$. 


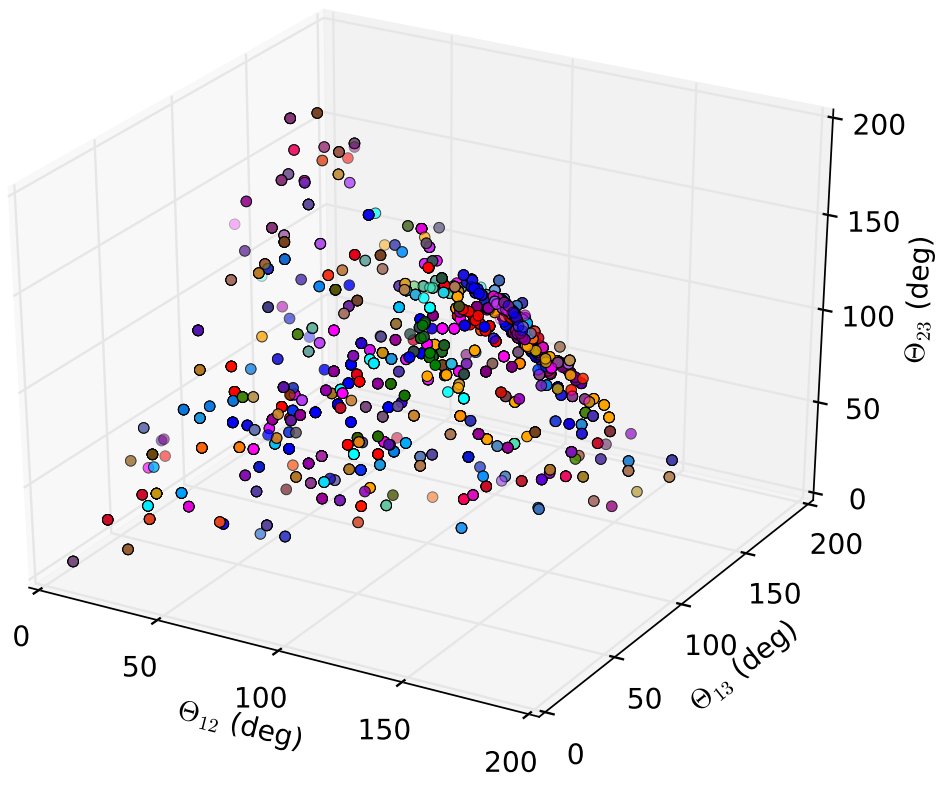

Figure 3.14: Initial magnetic moment configurations of candidates evaluated for $U=4$ $\mathrm{eV}, \mathrm{J}=0 \mathrm{eV}$ in $\mathrm{Mn}_{3} \mathrm{Pt}$. The $\mathrm{x}$-axis is the angle between the first and second $\mathrm{Mn}$ magnetic moments. The y-axis is the angle between the first and third Mn moment, and the z-axis is the angle between the second and third Mn moment. 


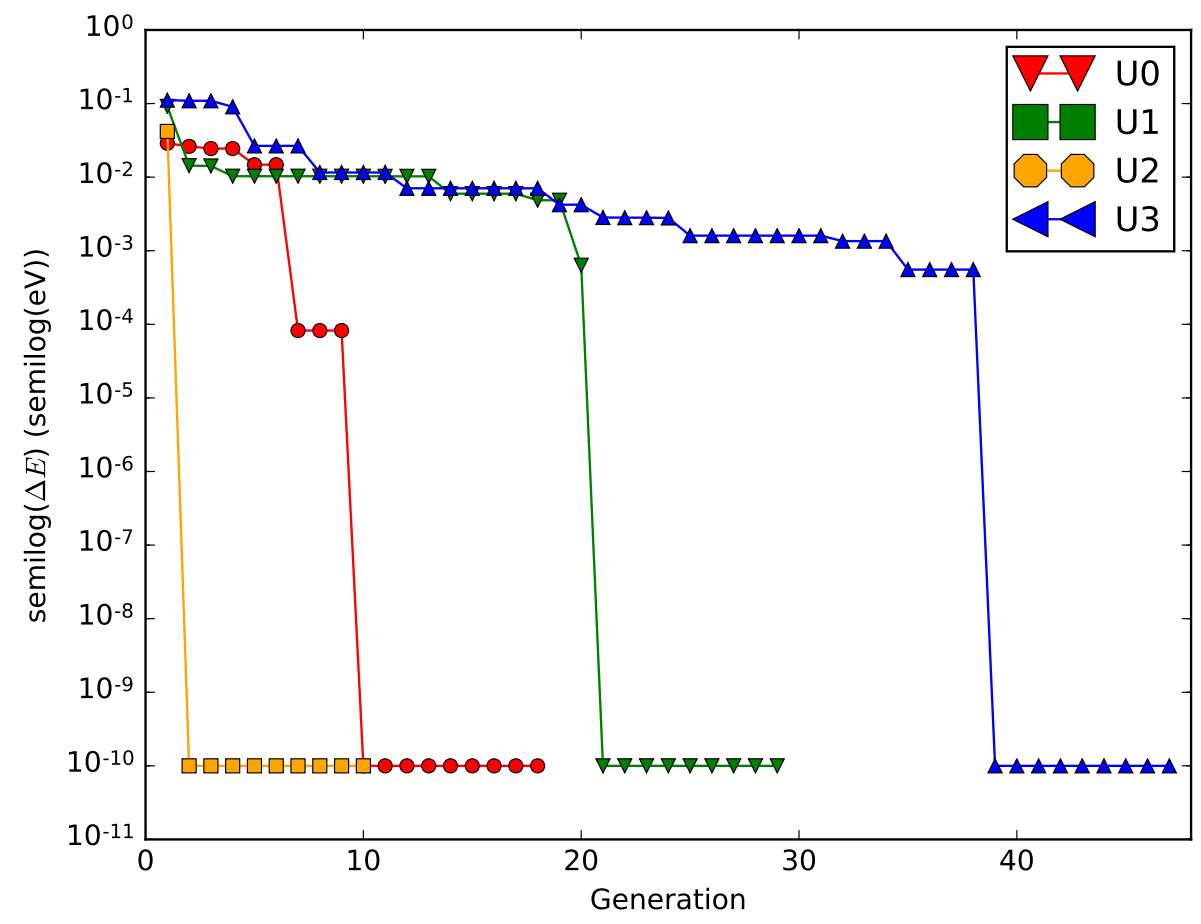

Figure 3.15: Energy difference between the final best candidate and the best candidate of each generation for both $\mathrm{J}=0$ in $\mathrm{Mn}_{3} \mathrm{Pt}$. This plot is logarithmic in order to show multiple values of $U$ on the same plot. The nine points corresponding to the final plateau for each candidate are the best candidate. Since this is a semilog plot, the energy difference has been selected as less than that of the energy tolerance used $\left(10^{-9} \mathrm{eV}\right)$ to avoid infinities in the graph. 


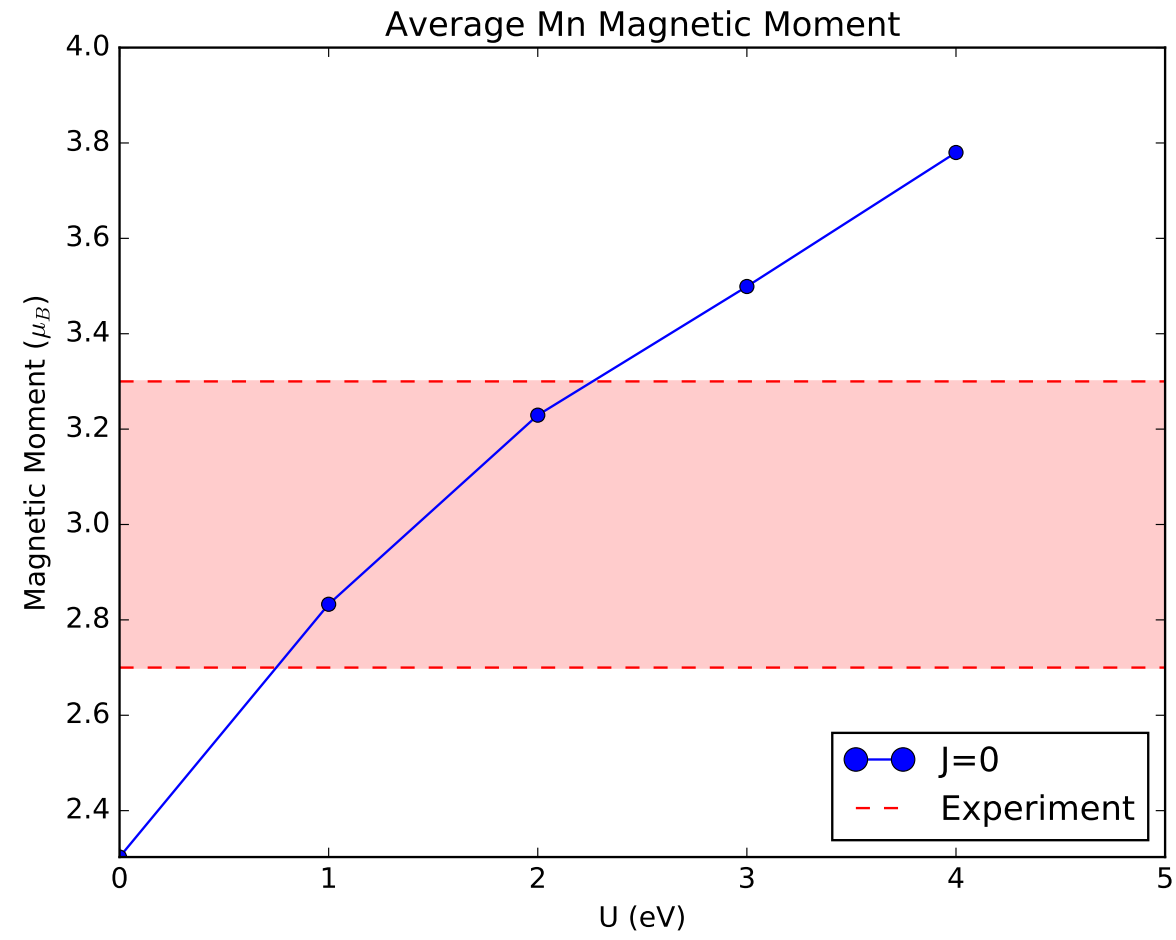

Figure 3.16: Magnetic moments of the lowest-energy candidates for $\mathrm{Mn}_{3} \mathrm{Pt}$. 


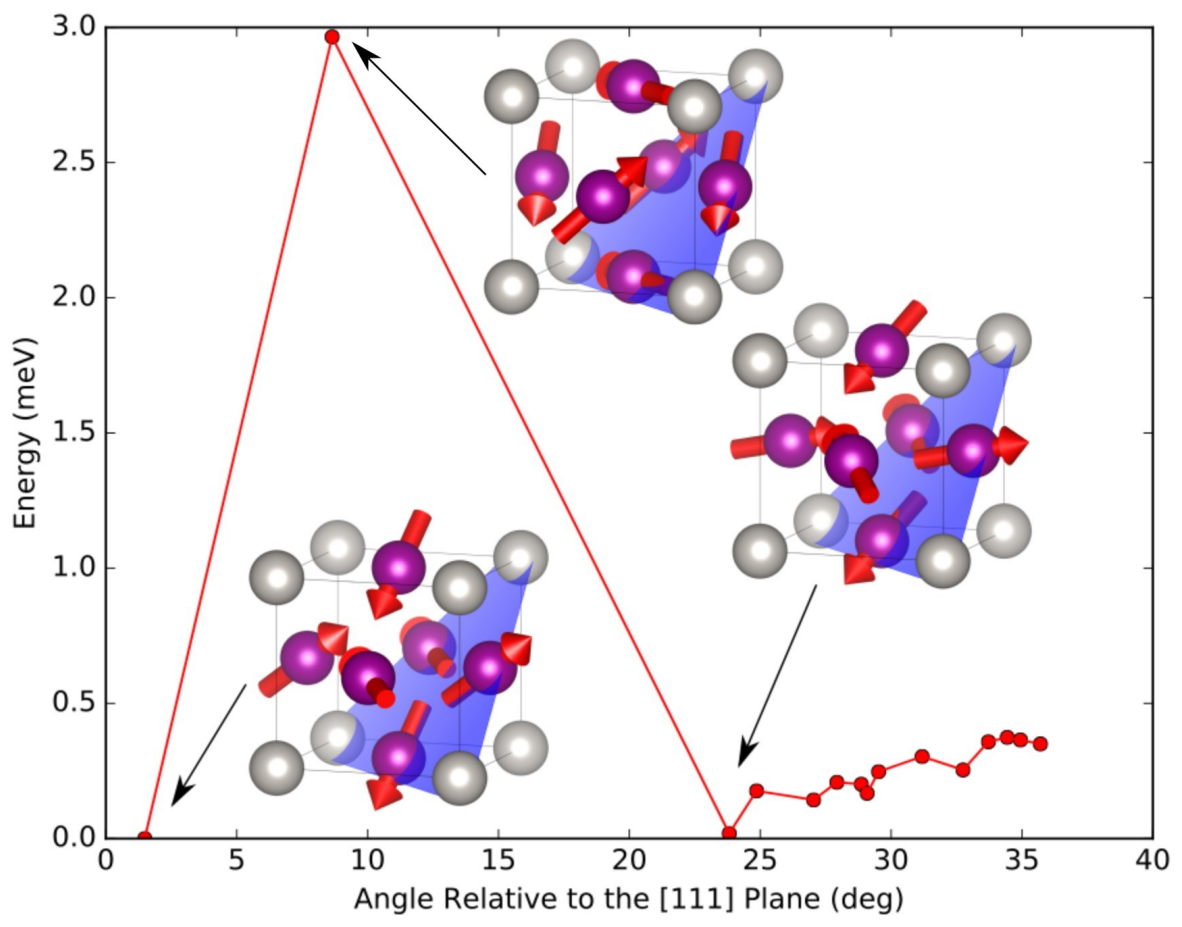

Figure 3.17: Relative energy of each candidate in terms of its orientation of the planes in which its magnetic moments lie after relaxation, relative to the [111] plane for $\mathrm{Mn}_{3} \mathrm{Pt}$. This are for the candidates in the final generation of the search for $\mathrm{U}=2.0, \mathrm{~J}=0.0$. There are many minima located near the lowest-energy candidate. The peak of the figure is 3 meV higher in energy than the lowest energy configuration. This higher energy candidate is identified as one of the three theoretically predicted orientations which agrees with experiment. The candidate which has an energy difference of $1 \mu \mathrm{eV}$ with the lowest energy candidate is identified as a degenerate state. 


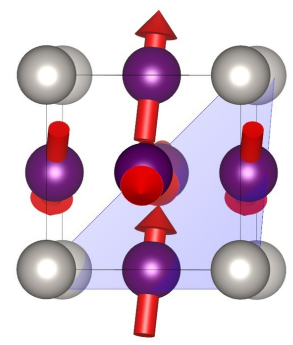

(a) $\mathrm{U}=1 \mathrm{eV}$

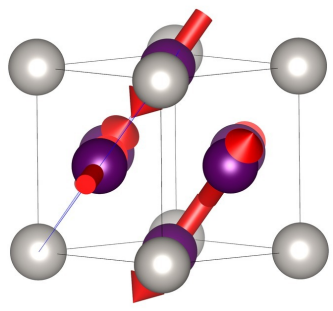

(c) $\mathrm{U}=2 \mathrm{eV}$

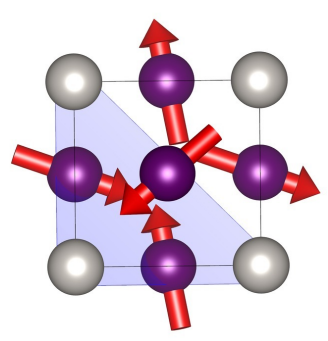

(b) $\mathrm{U}=1 \mathrm{eV}$

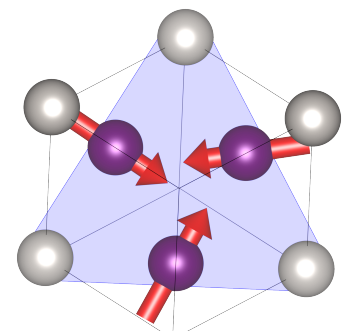

(d) $\mathrm{U}=2 \mathrm{eV}$

Figure 3.18: Magnetic moments of each low-energy candidate for $\mathrm{Mn}_{3} \mathrm{Pt}$. (a) and (c) display the orientation of the magnetic moments relative to the [111] plane. $U=2.0$ lies in this plane, $\mathrm{U}=1.0$ does not. (b) and (d) display the orientations of the magnetic moments within the plane they lie in. The result for $\mathrm{U}=2.0$ agrees with both experiment and previous theoretical results. 


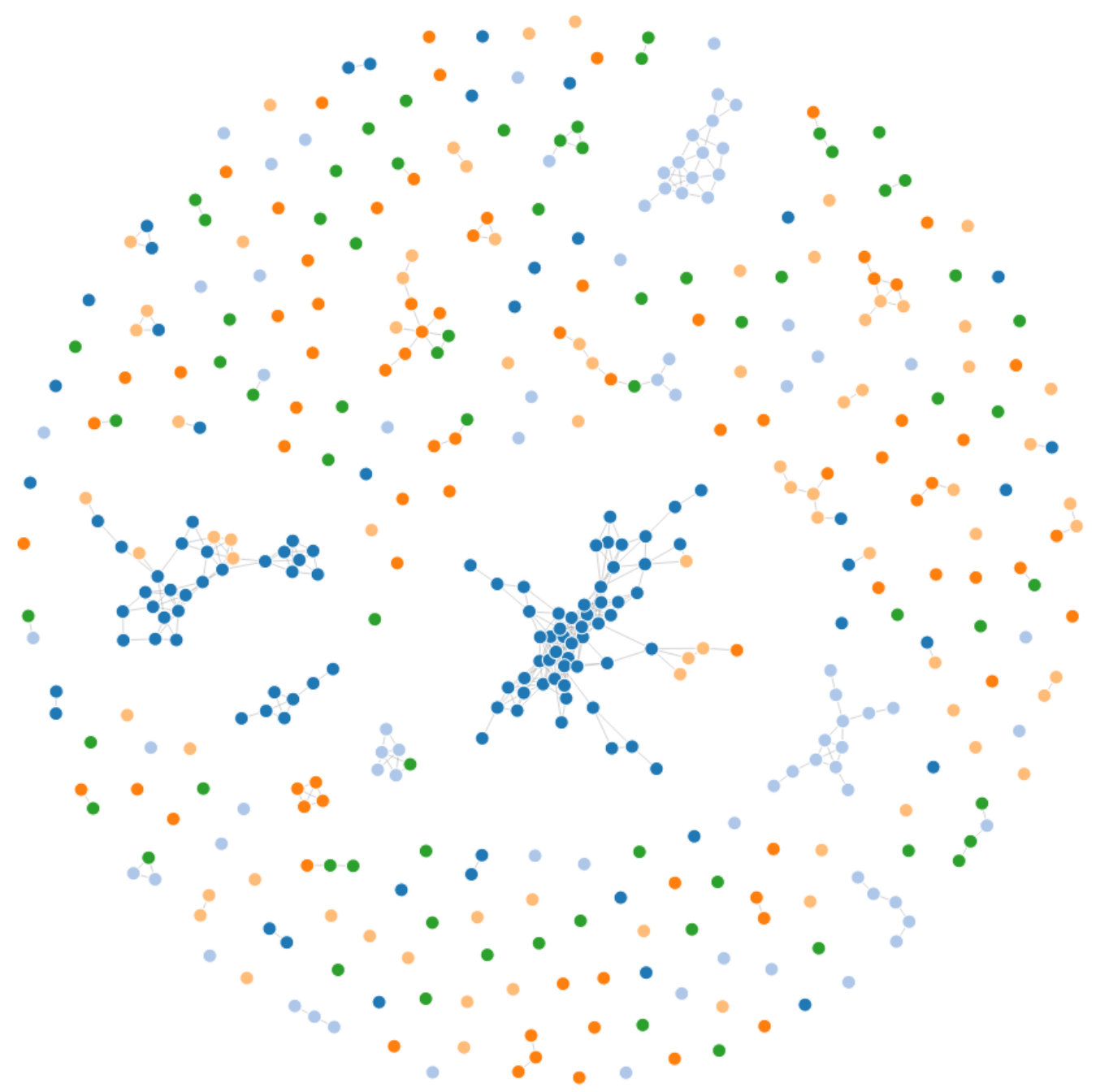

Figure 3.19: Network plot for the molecular system $\left(\mathrm{Mn}_{I V}\right)_{3} \mathrm{O}_{4} \mathrm{~L}_{4}\left(\mathrm{H}_{2} \mathrm{O}\right)$. As with the previous network plots, the distance cut-off for connected points is 0.3 radians. There are several regions which are highly connected, yet disconnected from one another. This indicates that FA explored multiple different minima. 


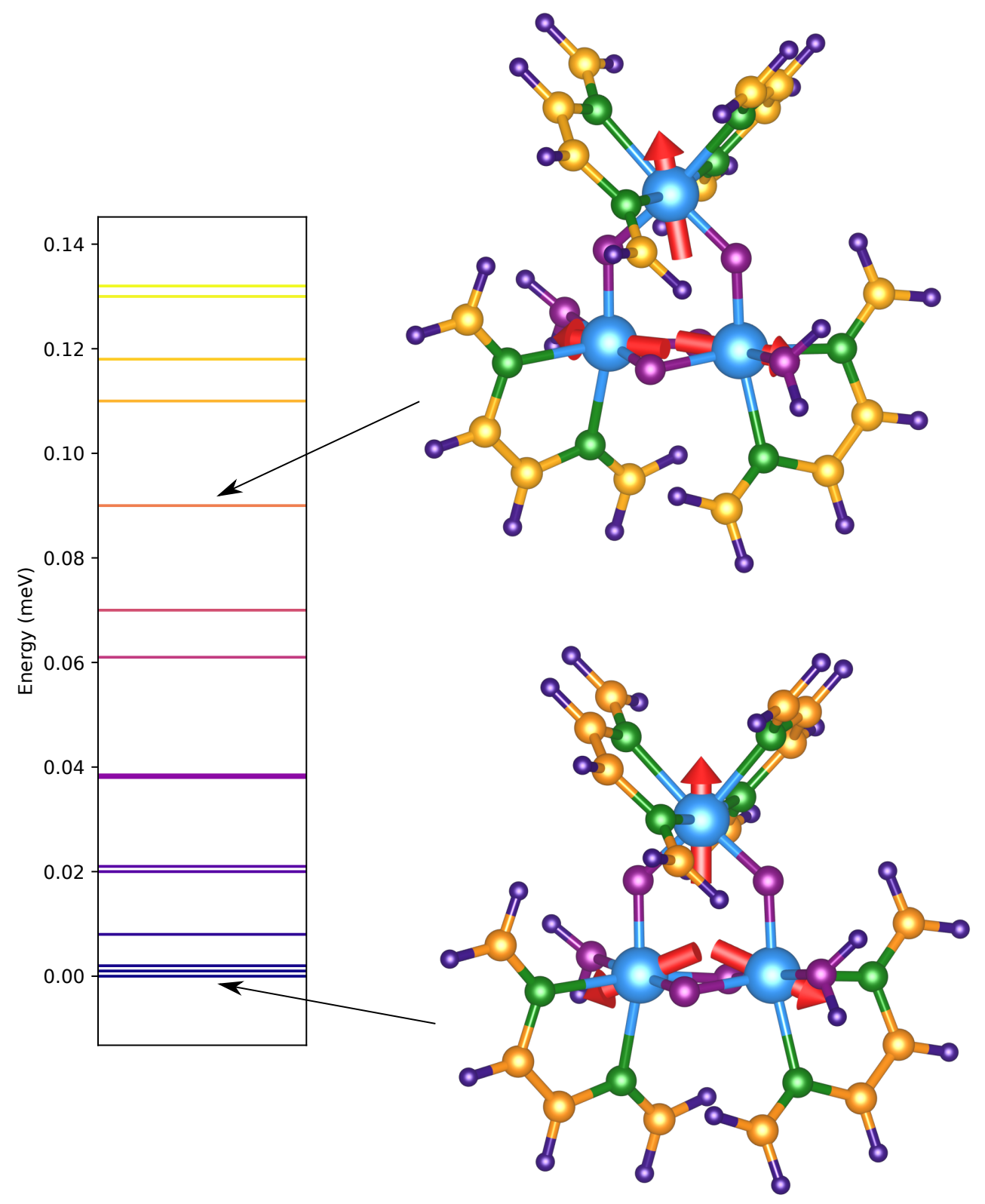

Figure 3.20: Energy differences between candidates in the final generation for $U=2 \mathrm{eV}$ $\mathrm{J}=0 \mathrm{eV}$ after relaxation. Each line corresponds to a different minima. The lowest-energy candidate has magnetic moment orientations and magntiudes which are in agreement with previous theoretical work. 


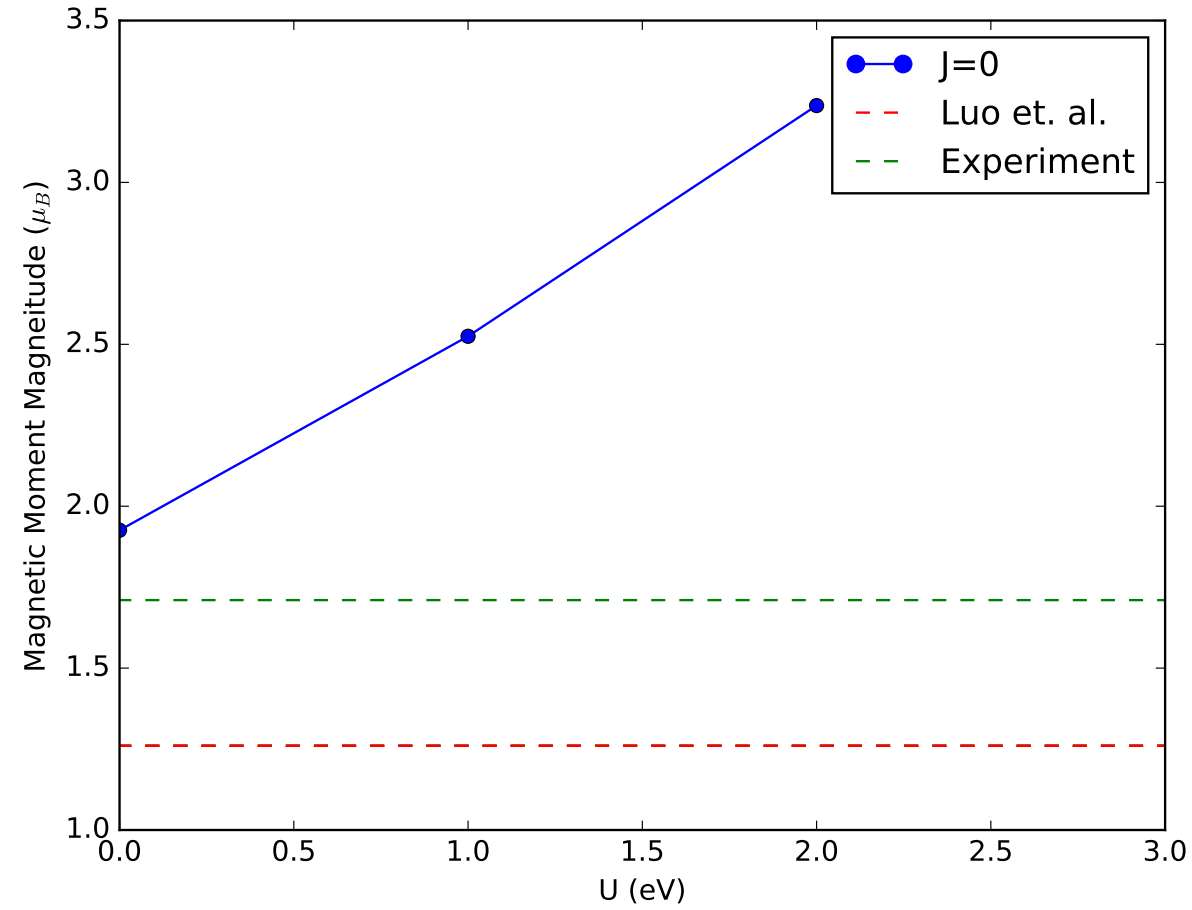

Figure 3.21: Average magnetic moment magnitude of the best candidate for all values of $\mathrm{U}$ and $\mathrm{J}$ considered for the molecular system. The dashed lines are the average magnetic moment value from previous theoretical and experimental work [70, 90] 


\section{Chapter 4}

\section{Orbital Search}

\subsection{Introduction}

As previously pointed out. the two most widely used approximations for exchangecorrelation potential in density functional theory (DFT), the linear density approximation (LDA) and the generalized gradient approximation (GGA), fail to capture the electronic structure of materials with partially filled d and f-electrons. [21, 22] This particular failure comes from the tendency of LDA/GGA to delocalize electrons due to the loss of self-interaction. Thus methods beyond ordinary DFT must be used to in order to correct this tendency to energetically favor delocalization. As with the magnetic moment search, here DFT+U is used again as its computational cost is comparable to ordinary DFT. This will be vital for the two test cases considered, as quickly generated results are necessary in order to assess the accuracy of FA when applied to the problem of orbital occupation. The use of search methods within DFT $+\mathrm{U}$ was previously shown to be necessary to locate the correct gorund state, as it was previously shown that the use of DFT+U generates a number of ficticious metastable states, [67, 27, 56, 3] (the same problem is also present for hybrid functionals or Hartree-Fock methods). The presence of these numerous metastable states makes the usual self-consistent algorithms to solve the DFT equation, such as the conjugate gradient algorithm, to fail in finding the orbital occupation that gives the global energy minimum of the system. If a calculation starts with an initial occupation which is close to one local minimum, it could converge towards this metastable state without exploring the other pos-

Adapted from Phys. Chem. Chem. Phys., 2019, 21, 21932 with permission from the PCCP Owner Societies. 
sibilities even though they are at lower energy. The other way around, in some cases these metastable states are close enough in energy to let the minimization algorithm to jump from one to the other but without the guarantee that the final reached state is the ground state.

One solution would then to sample the configuration space of all the allowed possible occupancy of the system such that the lowest energy orbital configuration can be found. For optimizing the orbital occupation matrix, quasi-annealing, dynamical mean field theory (DMFT) and occupation matrix control have all been used to try and solve this problem, but each has its drawbacks. In quasi-annealing, a fictitious fluctuation of the external potential is applied and gradually suppressed in order to reach the correct ground state. This method is analogous to gradually annealing the thermal kinetic energy in a classical system to reach local minima. This provides to a case stuck in a minima to gain enough energy to overcome the barrier between it and other minima. The main drawback of this method is that it struggles with locating the correct ground state in systems with many minima which are close in the calculated energy and width around this [35]; essentially it is too coarse in its search over low-energy states in the PES. DMFT consists of mapping the many-body lattice model, for example, the Hubbard model, to a local quantum impurity model, in this case, the Anderson impurity model, and subjects this to a self-consistency condition. Effectively, DMFT finds the Green's function for the impurity model which also reproduces the lattice model's Green's function through the interaction with an effective mean field. While this is an alternative method to using DFT, it is computationally demanding [36, 81], which limits its use in global search methods. This is not to say that the use of DMFT with FA is impossible, just that it could take months to generate results which would only takes weeks with DFT+U.

Here FA is used to determine the ground state occupancy of the d-electrons in the pervoskite-like $\mathrm{KCoF}_{3}$ and the f-electrons in the $\mathrm{AFM} \mathrm{UO}_{2}$. Essentially, FA builds upon the occupation matrix control method mentioned previously, but has several caveats. The first is that the occupation control method necessitates the generation and testing of several initial occupancies. For a single unit cell with one atom, this is a fairly trivial task. For example, with an atom that requires d-electron corrections, there are at most $\frac{5 !}{3 ! 2 !}=10$ possible configurations. This becomes much more complex whenever there are multiple atoms, or when supercells must be constructed to fully capture correlation effects, such as in antiferromagnets. In this regard, occupation matrix control is effectively a brute-force method which seeks to explore over every possible physical configuration of the $d$ (or f) electrons. Instead of trying to determine all possible configurations by hand, 
FA selectively explores differing orbital configurations on the potential energy surface (PES) of possible orbital configurations. This means that the algorithm itself is used to generate ever more energetically favorable solutions rather than trying to determine them ahead of time. While the basic theory behind FA has already been presented, how it is applied to generate differing orbital configurations will be described in further detail in the methodology section. After presenting the particulars of FA to the problem of orbital occupation, this method is applied to two test cases: the cubic perovskite $\mathrm{KCoF}_{3}$, which requires 3-d electron corrections, and $\mathrm{UO}_{2}$, which requires corrections to 5-f electrons.

\subsection{Methodology}

As with the magnetic moment case, here it will be described how FA is particularly applied to the problem of optimizing the orbital occupation matrix. As FA requires an initial random population of candidates, here this will be a collection of different orbital occupation matrices. In order to generate this differing occupation a unitary transformation is applied to a fixed occupation matrix. This initial fixed matrix is determined by the number of electrons that are being corrected, in the sense of DFT+U, in a given material system. The initially defined occupation matrix must have the property that the trace of this matrix is equal to the total number of electrons being corrected. Since the application of a unitary transformation does not change the trace, they in turn do not modify the total number of electrons in the considered system. However, the application of a unitary transformation will change the values of the diagonal and off-diagonal elements. It must be emphasized that the application of this transformation is done solely as a mathematical technique to generate new matrices; any physical interpretation of what occurs when performing a unitary transformation is not of importance here. The unitary transformation matrices can be generated by defining a series of independent parameters, as presented in the work by Hoffman et. al. [47] In general, any $\mathrm{N}$-dimensional unitary transformation can be represented by $\frac{1}{2} N(N-1)$ independent parameters. Here, these parameters have the following constraints:

$$
\begin{aligned}
\frac{-\pi}{2}<\theta_{k} & <\frac{\pi}{2} \\
-\pi<\theta_{N-1} & <\pi \\
\theta_{N} & =\frac{\pi}{2}
\end{aligned}
$$


, with $k=1,2,3, \ldots, N-2$.

In the case of $N=3$, these are the ordinary Euler angles, so these are referred to as generalized Euler angles. In the case of d-orbitals, the occupation matrix is of $5 \times 5$ dimension, which means ten Euler angles must be generated. For f-orbitals, the occupation matrix is $7 \times 7$, so 21 Euler angles are required. Once this transformation is applied to the initial fixed occupation, a new point is reached in orbital configuration space. A set of Euler angles are generated for every member of this initial population as they allow for the generation of differing initial occupations for each candidate. This also means that each candidate can be classified by its unique set of Euler angles. In fact, instead of the occupation matrices themselves, FA uses these Euler angles to determine distances between candidates and to generate new candidates. Once the Euler angles are defined, they can be used to generate the unitary transformation which in turn determines the orbital occupation of each candidate. Each candidate is then evaluated for how optimal of a solution it, by this, it's energy is evaluated. How "bright" a candidate determines how optimal it is as a solution, that is, how energetically favorable it is. As a reminder, just as in nature, multiple spatial distributions of fireflies can have equivalent brightness, meaning that FA is multi-modal; it can both explore multiple regions of the PES and have multiple solutions with the same total energy. For the same reasons as the magnetic moment optimization problem, this is precisely why it is well-suited to searching for optimal orbital occupations as degenerate occupations are not uncommon. Previously it was shown that the distance function, which determines the similarity between two candidates, had to be defined specifically for the property which was being optimized. For magnetic moment optimization, this distance as the average of the angles between magnetic moments on the same atom between pairs of candidates. Here, since each point on the PES can be characterized by a unique set of Euler angles, the average difference between the angles can be used to define this distance. The functional form of this measure is displayed in Equation 4.4. It should be noted that this is not entirely dissimilar in form in Equation 3.1. In fact, using angular distanced between differing candidates is not entirely uncommon in cases were Euclidean distance is a meaningless concept.

$$
r_{i j}=\frac{1}{N} \sum_{k}^{N}\left(\theta_{i}^{k}-\theta_{j}^{k}\right)
$$

Here, $N$ is the total number of Euler angles, $\theta_{i}^{k}$ is the $k$ th Euler angle for candidate $i$, and $\theta_{j}^{k}$ is the $k$ th Euler angle for candidate $j$. After this process, all of 
the moved candidates are promoted to the next generation along with the current lowest-energy solution. The process continues iteratively until a low-energy candidate survives for a specified number of generations. From previous work, ten generations provides an ideal compromise between accuracy and efficiency.

\subsection{Computational Details}

The ABINIT code [41, 40, 42, 99, 39] was used for all DFT calculations as it allows for both the use of DFT+U and for control of the orbital occupation. An input parameter, dmatpawu, is used to define the initial occupation of the corrected $\mathrm{d}$ or f-electrons.

In particular to the systems chosen as test cases, the LDA/GGA functionals of DFT predict that $\mathrm{KCoF}_{3}$ is metallic [84], which is why corrections to the electron correlation are necessary. As discussed, the performance of DFT+U as the method to correct the electron correlation in normal GGA functionals was explored. As this is an antiferromagnet, it has 4 magnetic cations in the basic unit cell. This already creates a large set of possible energy minima states based on the different $(J, U)$ parameters used in DFT+U. Since FA requires the calculation of multiple candidates simultaneously for at least ten generations, DFT+U appears the best option to efficiently test FA's accuracy.

The initial occupancy remain fixed for 20 electronic self consistent iterations. This is done in order to force the system onto the particular point on the PES which corresponds to the initially defined occupation matrix. After these initial 20 iterations, the occupation matrix is allowed to relax, which causes it to converge to the nearest low-energy state. With the implementation of FA to the magnetic moment optimization problem, it was seen that relaxation was necessary after the search was completed in $\mathrm{NiF}_{2}$. This was because the final low-energy candidate was not guaranteed to be in an energy minima, just that it was lower in energy than all other candidates. This would mean that the final candidate was in the basin of atraction for a minimum which was lower in energy than all other candidates, but had still not exactly reached this minimum. For this reason, it must be ensured that candidates are relaxed before their energy is determined. ABINIT allows for this as an input parameter in any $L D A+U$ calculation, further cementing its use for this problem. For $\mathrm{KCoF}_{3}$, an energy cutoff of $26 \mathrm{Ha}$ and a k-point mesh of $4 \times 4 \times 2$ was found to allow relaxation of the orbitals to a residual of the potential to a tolerance of $10^{-16}$. The same tolerance was reached for $\mathrm{UO}_{2}$ with an energy cutoff of $20 \mathrm{Ha}$ and a k-point grid of 6x6x6. Additionally, all calculations used the 


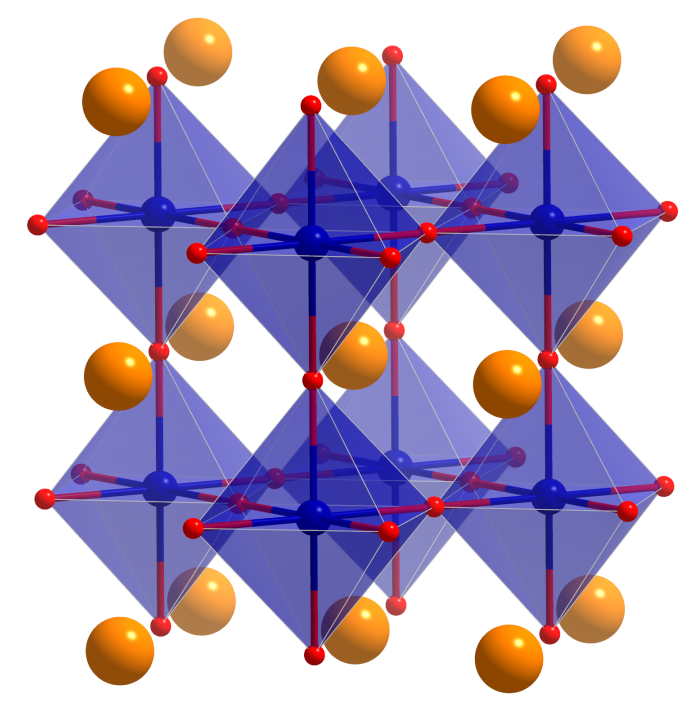

(a) $2 \times 2 \times 2$ supercell used for $\mathrm{KCoF}_{3}$.

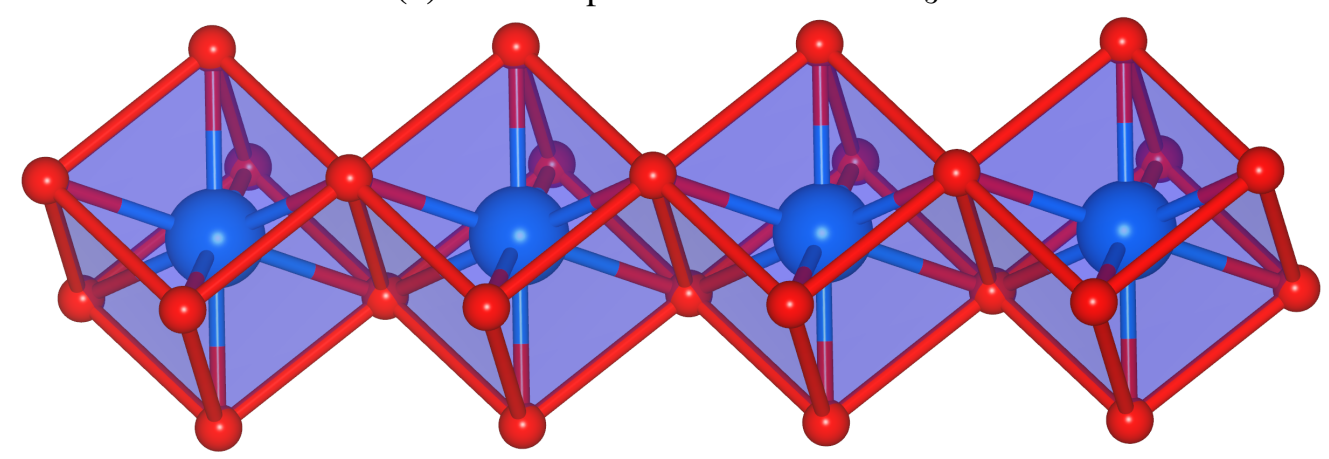

(b) $4 \mathrm{x} 1 \mathrm{x} 1$ supercell used for $\mathrm{UO}_{2}$

Figure 4.1: Supercells used for all FA calculations 
JTH v1.0 pseduopotentials with LDA exchange correlation. [92, 55]. For Co, 17 valence electrons were considered, and for U 14 were considered. ABINIT allows for straightforward control of the occupancy matrices with the use of the dmatpawu variable. [39] For d-electron corrections, these are 5x5 matrices for each atom which requires d-orbital corrections using the DFT+U method, in particular Liechtenstein's rotational invariant method + FLL double counting corrections [5, 68]. For f-electron corrections, $7 \times 7$ matrices for each $U$ atom are used. The initial values for these matrices were determined by considering the total number of electrons per corrected atom, and defining diagonal matrices such that the trace of these matrices is equal to the total number of electrons in the f orbitals. FA handles the generation of new occupation matrices for each random candidate by applying selecting random Euler angles and applying the corresponding unitary transformation to each of these diagonal matrices.

After the low-energy candidates for each $U$ and $J$ were found, the magnetic exchange couplings were calculated as function of the $U$ parameter. For each candidate $\mathrm{KCoF}_{3}$ state, the magnetic exchange interaction parameters are calculated using the magnetic force theorem (MFT) [69], which use local spin rotation as a perturbation and calculate the response with Green's function method. To get the local perturbation, Maximally localized Wannier functions (MLWF) [76] for Co $3 d$ and F $2 p$ orbitals were constructed and the Kohn-Sham Hamiltonian from Abinit is mapped onto this basis set. Thus MFT can be applied to the Hamiltonian and get the exchange parameters [58].

\subsection{Results}

\subsection{1 $\mathrm{KCoF}_{3}$}

The first system chosen to show the application of FA to orbital occupations is the cubic perovskite $\mathrm{KCoF}_{3}$ with space group $P m-3 m$, an anti-ferromagnetic Mott insulator [57, 48]. The cell parameters are $a=b=c=4.105 \AA$. The Wyckoff positions for the cell used are listed in Table 4.1. A $2 \times 2 \times 2$ supercell built from this unit cell is used for all calculations. The structures for both $\mathrm{KCoF}_{3}$ and $\mathrm{UO}_{2}$ are taken from the Materials Project. [52]

The $\mathrm{F}$ atoms octahedron crystal field $(\mathrm{CF})$ split the Co 3d-orbitals into three degenerate $\mathrm{t}_{2 g}\left(\mathrm{~d}_{x y}, \mathrm{~d}_{x z}\right.$, and $\left.\mathrm{d}_{y z}\right)$ and two $\mathrm{e}_{g}$ orbitals $\left(\mathrm{d}_{3 z^{2}-r^{2}}\right.$, and $\left.\mathrm{d}_{x^{2}-y^{2}}\right)$ [80]. In general, for $\mathrm{Co}^{2+}$ in an octahedral crystal field, the CF splitting $\Delta$ is small, which yields the high-spin occupancy $\mathrm{t}_{2 g}^{5} \mathrm{e}_{g}^{2}$ with a nominal magnetic moment of $3 \mu_{B}$. If 
$\mathrm{KCoF}_{3} \mathrm{Pm}-3 m$

\begin{tabular}{|c|c|c|c|c|}
\hline Atom & Wyckoff Position & $\mathrm{x}$ & $\mathrm{y}$ & $\mathrm{z}$ \\
\hline $\mathrm{K} 1$ & $\mathrm{a}$ & 0.00 & 0.00 & 0.00 \\
Co2 & $\mathrm{b}$ & 0.50 & 0.50 & 0.50 \\
F1 & $3 \mathrm{c}$ & 0.00 & 0.50 & 0.50 \\
\hline
\end{tabular}

Table 4.1: Structural information for $\mathrm{KCoF}_{3}$. Coordinates for each atom are in reduced coordinates.

\begin{tabular}{|c|c|c|}
\hline $\mathrm{U}(\mathrm{eV})$ & $\mathrm{J}(\mathrm{eV})$ & Band Gap (eV) \\
\hline 1 & 0 & 0.67 \\
2 & 0 & 0.78 \\
3 & 0 & 2.31 \\
4 & 0 & 1.90 \\
\hline \multicolumn{2}{|c|}{ Experiment [73] } & $2.1 \pm 0.2$ \\
\hline
\end{tabular}

Table 4.2: Band gaps for the best candidate for all values of $U$ and $J$ considered for $\mathrm{KCoF}_{3}$.

$\Delta$ is large, the low-spin configuration is favored with the occupancy $\mathrm{t}_{2 g}^{6} \mathrm{e}_{g}^{1}$ and a nominal magnetic moment of $1 \mu_{B}$. Experimentally, the magnetic moment of $\mathrm{Co}$ in $\mathrm{KCoF}_{3}$ is $3.33 \mu_{B}$ [80], which corresponds to the high-spin configuration, and its band gap is $2.1 \pm 0.2 \mathrm{eV}$.[73] Table 4.2] lists the band gaps for the bets candidates for each value of $U$ and $\mathrm{J}$ considered. Only $\mathrm{U}=4 \mathrm{eV} \mathrm{J}=0 \mathrm{eV}$ is within the range of reported experimental values. Later it will be shown that the orbital occupancy is sensitive to the choice of values for $U$ and $J$. These differing occupations can lead to different physical properties, which is why these band gaps do not vary monotonically.

As with the magnetic moment test cases, to show that FA can find these states, as well as any metastable states, the searcher must satisfy the following:

1. The lowest-energy candidate improves as the generation increases.

2. The diversity of candidates increases as the generation increases.

While the use of any metaheuristic can never guarantee that the lowest-energy solution is the ground state, it should at the very least approximate it or approach it. For the three magnetic test cases that FA was applied to, it can find lowenergy candidates with properties that are in agreement with the ground-state from 
previous experimental results and theoretical calculations. This means that there should be an improvement of the lowest-energy candidate as the searcher explores more of the PES. To demonstrate the first point, the energy differences between the of the low-energy candidate and the best candidate can be plotted as a function of generation, as shown in Figure 4.2 .

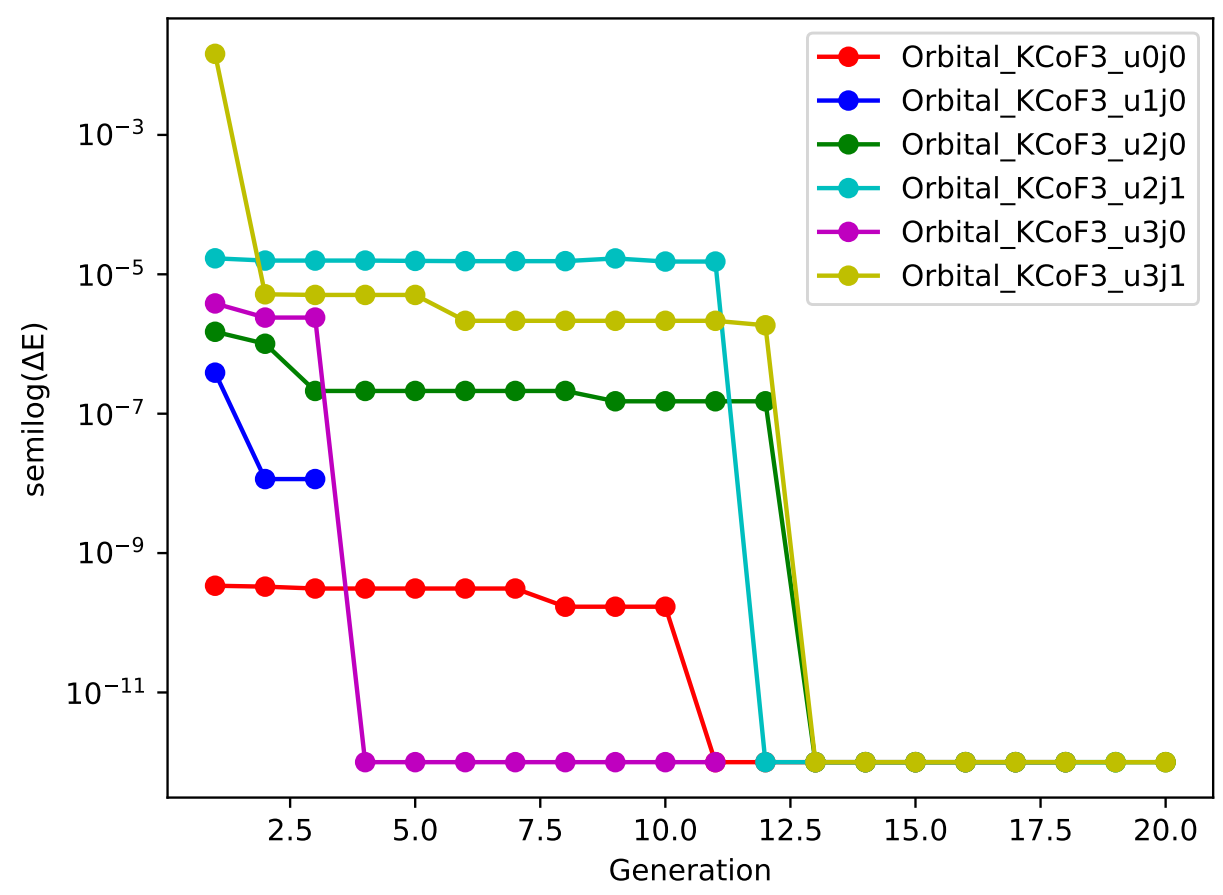

Figure 4.2: Semilog plot of the energy of the best candidate in each generation relative to the energy of the lowest energy candidate found. As the semilog of zero diverges, an energy difference of $10^{-12}$ Ha was used for candidates which have the same energy as this candidate, as this value of energy is at least two orders of magnitude lower than the energy difference between any candidate with this lowest energy candidate.

As this is a semilog plot, the best candidate cannot be shown, as the semilog of zero is undefined, and approaches infinity. To rectify this, the points corresponding to the bets candidate are automatically set to $10^{-12}$, as this is significantly lower than any order of magnitude decrease as the searcher evolves. Discounting this, it can be seen from this figure that the candidates mostly improve by two- 
to-three orders of magnitude. As a secondary point, the the best candidate should survive for 10 generations to prove that the stabilization limit is reached.

To demonstrate the second point, a network graph is generated in the exact same fashion as in the magnetic test cases. Just as before, this network graph represents the areas of the PES which have been explored by FA. The network plot is shown in Figure 4.3 for $\mathrm{U}=3 \mathrm{eV}, \mathrm{J}=1 \mathrm{eV}$. Each point corresponds to a unique candidate, and each candidate only forms connections to candidates which are less a distance tolerance of 0.3 radians. For all calculations the distance is found by evaluating the average difference between the Euler angles for two candidates, as defined in Equation 4.4. Thus, the purpose of this tolerance is to restrict each candidate to the region local to it on the potential energy surface (PES), and to determine what other candidates are in this local region. The reasoning behind this is two-fold: First, FA operates through modifying candidates to search in the area around a minima; essentially once FA locates a candidate close to minima, in subsequent generations this candidate is modified to explore the region which is local to it.

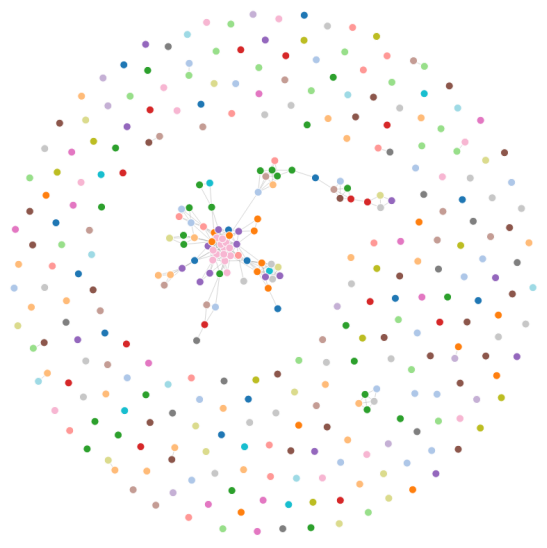

(a)

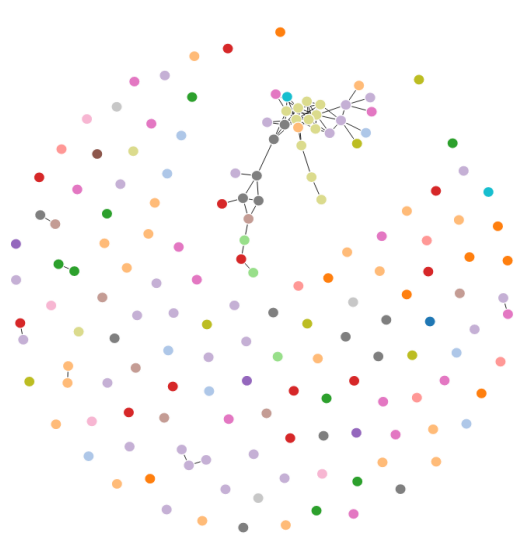

(b)

Figure 4.3: Network plot for the connectivity between all candidates found by the Firefly searcher for (a) $\mathrm{KCoF}_{3}$ and (b) $\mathrm{UO}_{2}$. Candidates which are connected are less than a predefined distance tolerance of 0.3 , which means the average difference between the two candidate's Euler angles is less than 0.3 radians. From this plot, there is a region of highly connected candidates, indicating that the searcher is targeted.

If the distance tolerance is set low enough for this plot, then candidates which lie in a minima on the PES will only be connected to other candidates which lie in 
the basin of that minima, which in turn also shows that FA is targeted. Second, the random candidates which replace identical candidates generally lie on the PES at points that are far away from the minima. Restricting this distance tolerance to the appropriate value means these random points will be disconnected. There are several regions in the plot where points are clumped together; these are candidates which lie close to a local minima on the PES. If the average difference is less than 0.1 radians, then the two candidates are considered identical. For this network plot, two nodes are connected if this average angle is greater than 0.1 radians and less than 0.3 radians, as the distance tolerance must be greater than the threshold for identical candidates, but must also remain small to denote candidates which lie close to one another on the PES. With this in mind, it can be seen from Figure 4.3 that the search by FA is targeted.

Additionally, there are many disconnected points, which means identical candidates are being replaced as the searcher evolves.

The lowest energy candidate for non-zero values of $U$ and $J$ always had the high-spin configuration $\mathrm{t}_{2 g}^{5} \mathrm{e}_{g}^{2}$, as expected for this crystal. Unsurprisingly, for $\mathrm{U}=$ $0 \mathrm{eV}$ and $\mathrm{J}=0 \mathrm{eV}$, the electrons are delocalized. The energy differences between all candidates in the final generation of $\mathrm{U}=4 \mathrm{eV}, \mathrm{J}=0 \mathrm{eV}$ is displayed in Figure 4.4. The orbitals for the lowest-energy candidate is displayed in Figure 4.5. This plot was generated using the Wannier90 software package, which is used to determine the maximally-localised Wannier functions. It was used as ABINIT implements the generation of the necessary input files for the Wannier90 calculation. There is a two-fold degeneracy found for the lowest-energy candidate; there are two candidates with differing occupations with the same energy. While there should be three degenerate occupancies by symmetry, the failure of FA to locate all three is not a failure of the algorithm. These two-candidates appear for $U \neq 0$. In both of these candidates, the majority spin channel has 5 electrons occupying all possible d-orbital; they differ in the minority spin channel. In one case, one electron occupies the $x y$ orbital, the other in a linear combination of $x z$ and $x y$. In the other case, one electron occupies $x z$ while the other is in a linear combination of $x y$ and $y z$. For the linear combination of orbitals, there is a 50-50 contribution from each singular orbital which comprises it. These are shown in Figure 4.5.

In addition, the value of the magnetic exchange constants was calculated for the lowest energy candidate for all values of $U$ and $J$ considered. The values along several spatial directions, $\mathbf{J}_{x}=\mathbf{a}+\mathbf{b}, \mathbf{J}_{y}=-\mathbf{a}+\mathbf{b}$, and $\mathbf{J}_{z}=\mathbf{c}$, are listed in Table 4.3 .

Both $\mathrm{U}=3 \mathrm{eV} \mathrm{J}=0 \mathrm{eV}$ and $\mathrm{U}=4 \mathrm{eV} \mathrm{J}=0 \mathrm{eV}$ have magnetic exchange values within the range of accepted experimental values, which are $1.2 \mathrm{meV}$ to $4.5 \mathrm{meV}$. 

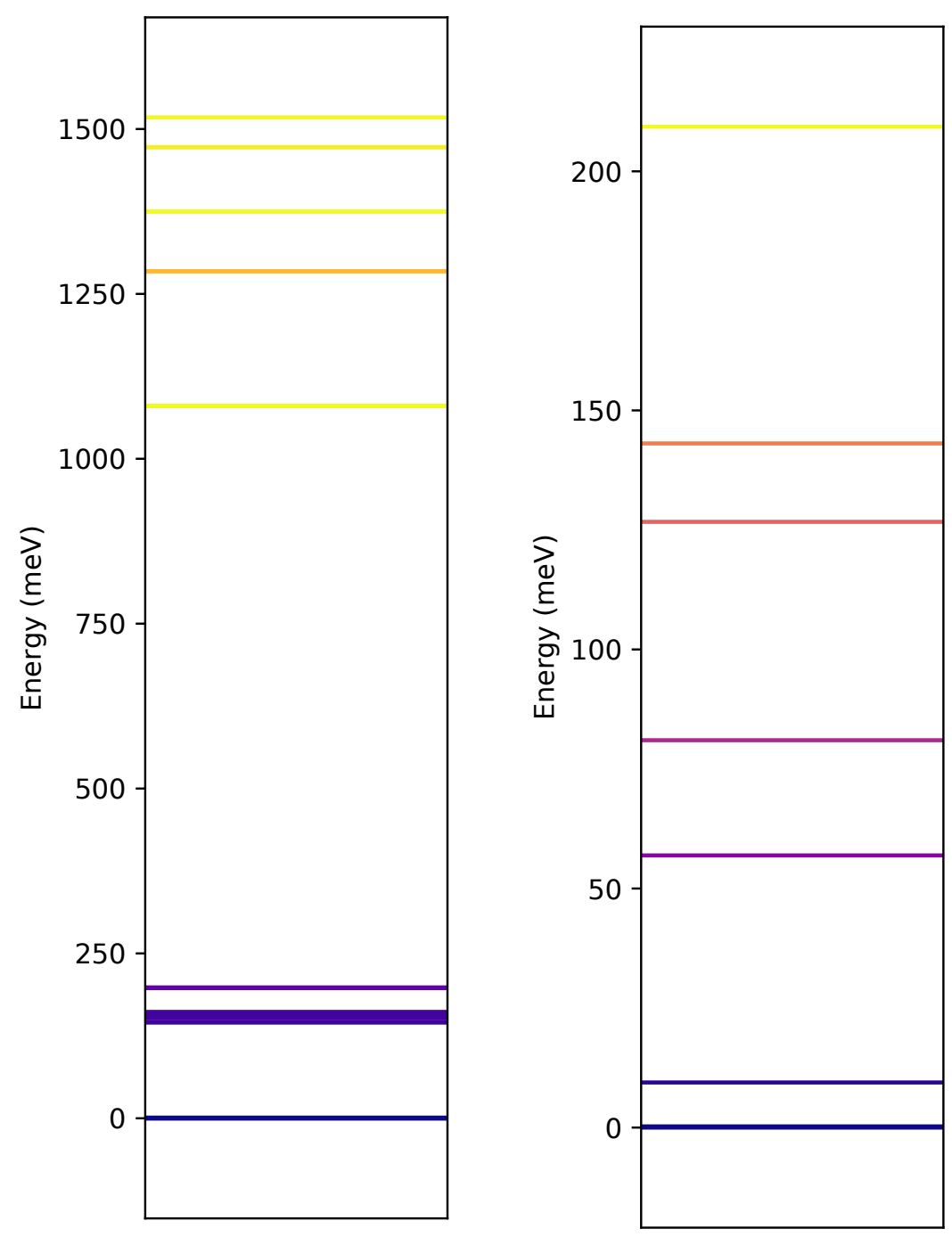

Figure 4.4: Energy differences between all candidates in the final generation with respect to the best candidate for (Left) $\mathrm{KCoF}_{3}\left(\mathrm{U}=4 \mathrm{eV} \mathrm{J}=0 \mathrm{eV}\right.$ ) and (Right) $\mathrm{UO}_{2}(\mathrm{U}=4 \mathrm{eV} \mathrm{J}=1$ $\mathrm{eV})$. 


\begin{tabular}{|c|c|c|c|c|}
\hline $\mathrm{U}(\mathrm{eV})$ & $\mathrm{J}(\mathrm{eV})$ & $J_{x}(\mathrm{meV})$ & $J_{y}(\mathrm{meV})$ & $J_{z}(\mathrm{meV})$ \\
\hline 0 & 0 & -12.02 & -11.456 & -11.46 \\
1 & 0 & -12.75 & -9.37 & -11.73 \\
2 & 0 & -10.99 & -8.24 & -10.16 \\
2 & 1 & -8.20 & -8.41 & -8.41 \\
3 & 0 & -5.34 & -3.82 & -1.47 \\
3 & 1 & -6.36 & -10.33 & -10.33 \\
4 & 0 & -4.78 & -1.31 & -3.47 \\
Experiment][48] & & 1.2 \\
\multicolumn{2}{|l|}{ Experiment][14] } & & 4.5 \\
\hline
\end{tabular}

Table 4.3: Magnetic exchange constants for the lowest-energy candidates of $\mathrm{KCoF}_{3}$. $\mathrm{U}=3 \mathrm{eV} \mathrm{J}=1 \mathrm{eV}$ and $\mathrm{U}=4 \mathrm{eV} \mathrm{J}=0 \mathrm{eV}$ have values within the range of those previously reported. The reported values are spatial averages.

$[14,48,78]$. However, for the calculated values of the band gap and the magnetic exchange, $\mathrm{U}=4 \mathrm{eV} \mathrm{J}=0 \mathrm{eV}$ is the best set of parameters to reproduce most of the experimental values together. For this test case, FA was able to locate lowenergy candidates that agree with theoretical predictions about the occupancy, and experimental properties of the ground-state.
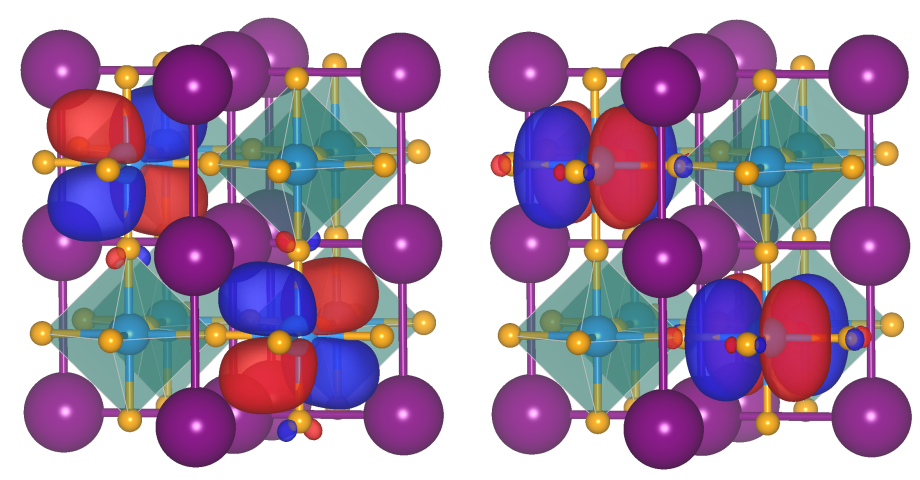

Figure 4.5: Occupied orbitals of the minority spin-channel for the best candidate for $U=4$ $\mathrm{eV} \mathrm{J}=0 \mathrm{eV}$. The Wannier function for a single Co is identical on each site. 


\subsection{2 $\mathrm{UO}_{2}$}

The second system chosen as a test case for FA is $F m$ - $3 m$ uranium dioxide $\left(\mathrm{UO}_{2}\right)$. $\mathrm{UO}_{2}$ is the standard fuel used in pressurized water nuclear reactors. Due to this, it has been extensively studied both experimentally and theoretically. Both LDA and GGA predict that $\mathrm{UO}_{2}$ is a metallic ferromagnetic, but it is known experimentally to be an antiferromagnetic Mott insulator [103]. While $\mathrm{UO}_{2}$ has been shown to from both experiment and calculation [15, 19] to have $3 \mathbf{K}$ antiferromagnetic (AFM) order, 1K AFM order is assumed, as the uranium atom's spins change sign along the z-axis. This changes the point symmetry group of $\mathrm{UO}_{2}$ from $\mathrm{O}_{h}$ to $\mathrm{D}_{4 h}$. The crystal field then splits the $5 \mathrm{f}$ orbitals of uranium into two two-fold degenerate levels, $\mathrm{E}_{u}$, and three non-degenerate levels, $\mathrm{A}_{2 u}, \mathrm{~B}_{1 u}$, and $\mathrm{B}_{2 u}$. In $\mathrm{UO}_{2}$, only two electrons are in the $5_{f}$ shell, so the initial diagonal matrix from which all other candidates are generated will have a trace equal to two. Here the primitive cell is used. The cell parameters are $a=b=c=3.83 \AA$. The Wyckoff positions for the cell used are listed in Table 4.4. Since there is only one uranium atom in the primitive cell, a $4 \times 1 \times 1$ supercell is constructed to capture the AFM nature of UO2. This supercell is used for all calculations.

$\mathrm{UO}_{2} \boldsymbol{F m - 3 m}$

\begin{tabular}{|c|c|c|c|c|}
\hline Atom & $\begin{array}{c}\text { Wyckoff } \\
\text { Position }\end{array}$ & $\mathrm{x}$ & $\mathrm{y}$ & $\mathrm{z}$ \\
\hline $\mathrm{U} 1$ & $\mathrm{~b}$ & 0.50 & 0.50 & 0.50 \\
O1 & 2c & 0.75 & 0.75 & 0.75 \\
\hline
\end{tabular}

Table 4.4: Structural information for the unit cell of $\mathrm{UO}_{2}$. Coordinates for each atom are in reduced coordinates. A $4 \times 1 \times 1$ supercell was generated from this cell to capture the AFM nature of $\mathrm{UO}_{2}$.

As in the case of $\mathrm{KCoF}_{3}$, it must be shown that the lowest-energy candidate converges with respect to the generation number, and that a the PES is adequately explored. Unlike in the case of $\mathrm{KCoF}_{3}$, there are several computational studies [2, 28] which have been performed to determine the orbital occupations of the ground state of $\mathrm{UO}_{2}$, so the results can be directly compared to previous computational studies. As a word of caution, however, in each of these studies the methods used are not exhaustive, and it is emphasized that there were no guarantee that the full orbital occupation space is explored.

In the work performed by Dorado et. al.[28], the occupation matrix control method was used to find the lowest energy state in $\mathrm{UO}_{2}$ with fluorite-structure with 
a twelve atom cell. They also presumed 1K AFM ordering. With the presumption of no symmetry (therefore allowing the cubic symmetry of to be broken), they found two degenerate low-energy states and ten metastable states. Using values $U=4.50 \mathrm{eV}$ and $J=0.5 \mathrm{eV}$, Dorado et. al. relaxed $\mathrm{UO}_{2}$ to the ground state when the occupancy matrices were initially imposed to have integer occupancy for the $m=-1, m=0$ or the $m=0, m=1$ orbitals. These initially defined occupation matrices are then relaxed. These two cases had band gaps which where comparable to the experimentally reported value of $2 \mathrm{eV}$. However, as previously stated, it is not guaranteed that this corresponds to be the true ground state. This is emphasized by the energy differences found between the lowest energy state and the next highest energy state, which differed only by $0.02 \mathrm{eV}$.

This makes $\mathrm{UO}_{2}$ an ideal complex test case for FA to explore the multiple $f$ orbital occupation minima. As previously shown for $\mathrm{KCoF}_{3}$, it must be shown that the lowest-energy candidate improves in each subsequent generation and a significant area of the PES is explored. Unlike the previously cited work, combinations of $\mathrm{U}=2,3,4$ and $5 \mathrm{eV}$ with $\mathrm{J}=1$ and $2 \mathrm{eV}$ are used, with $U>J$.

In Figure 4.6 the convergence of the lowest-energy candidate found with respect to the generation for all values of $U$ and $J$ considered for $\mathrm{UO}_{2}$ is shown. Just as with $\mathrm{KCoF}_{3}$, the lowest-energy candidate improves by 2-3 orders of magnitude.

In Figure 4.3 the network plot for $\mathrm{U}=4 \mathrm{eV} \mathrm{J}=1 \mathrm{eV}$ is displayed. What is striking about this plot is that the explored region is highly connected: each of these nodes has Euler angles which only differ by 0.3 radians on average. This seems to be in line with the idea that there are many low-lying states, brought upon by the use of LDA+U. Additionally, this highly-connected region is surrounded by many disconnected candidates, which correspond to random candidates, either discarded or changed significantly (i.e. with Euler angles differed from every other candidate by more than 0.3 radians).

For all values of $U$ and $J$ considered, all of the metastable states previously reported were located by FA. Furthermore, since the search is performed over several different pairs of values, the low-energy states can be compared across the selected values of $U$ and $J$. For the values of $U$ and $J$ considered the low-energy state found by FA has a different occupation than what is reported previously. This indicates that for $\mathrm{UO}_{2}$ the particular orbitals occupied depends on the choice of $\mathrm{U}$ and $\mathrm{J}$. Unlike $\mathrm{KCoF}_{3}, \mathrm{UO}_{2}$ shows that the variations in the occupancy of the electrons differ across the different values of $U$ considered. The variation of the occupancy across $\mathrm{U}$ for $\mathrm{J}=1 \mathrm{eV}$ is shown in Figure 4.8. For the values of $\mathrm{U}$ and $\mathrm{J}$ which are the most similar to Dorado et. al., which in their case are $\mathrm{U}=4$, $\mathrm{J}=0.5$, it was found that for $\mathrm{U}=4 \mathrm{eV} \mathrm{J}=1 \mathrm{eV}$ that the two electrons occupy $\mathrm{f}_{y z^{2}}$ 


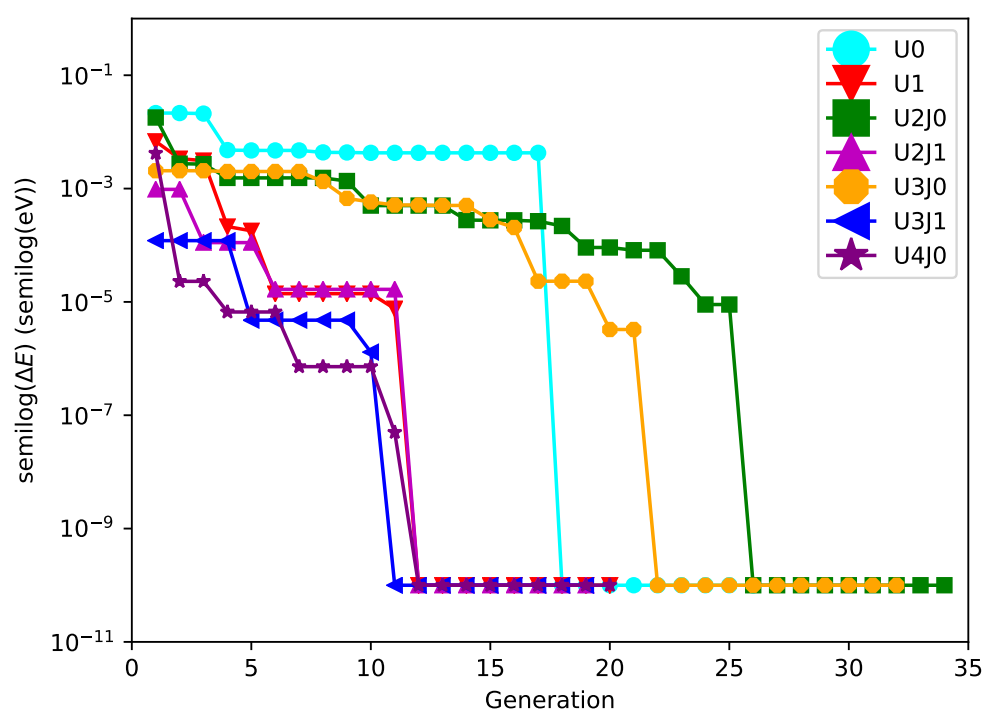

Figure 4.6: Semilog plot of the energy of the best candidate in each generation relative to the energy of the lowest energy candidate found for all values of $U$ and $J$ considered for $\mathrm{UO}_{2}$. As the semilog of zero diverges, an energy difference of $10^{-12} \mathrm{Ha}$ was used for candidates which have the same energy as this candidate, as this value of energy is at least two orders of magnitude lower than the energy difference between any candidate with this lowest energy candidate.

and a linear combination of $\mathrm{f}_{z\left(x^{2}-y^{2}\right)}$ and $\mathrm{f}_{x y z}$. In comparison, the ground state found by Dorado et. al. was $\mathrm{f}_{z\left(x^{2}-y^{2}\right)}$ and a linear combination of $\mathrm{f}_{x\left(x^{2}-3 y^{2}\right)}$ and $\mathrm{f}_{z^{3}}$. However, care must be taken here as the values of $\mathrm{U}$ and $\mathrm{J}$ are not identical, as the low-energy occupation for $\mathrm{UO}_{2}$ is sensitive to the choice of $\mathrm{U}$ and $\mathrm{J}$ (Figure 4.8). In their work, this state was found when non-diagonal occupation matrices were considered, and is $0.02 \mathrm{eV}$ lower in energy than the ground state found when only integer occupations were considered. The reported low-energy state was also found when integer occupations were enforced, with the two electrons occupying $\mathrm{f}_{x y z}$ and $\mathrm{f}_{x z^{2}}$, and similarly it is one of the metastable states found with FA. For $\mathrm{U}=4 \mathrm{eV}, \mathrm{J}=1 \mathrm{eV}$, this state is $1.98 \mathrm{eV}$ higher in energy than the low-energy state found with FA, indicating that the low-energy state found with FA is even lower in energy than that previously reported, which proves that using a metaheuristic search of occupation matrices could be more powerful than "hand-made" techniques. 

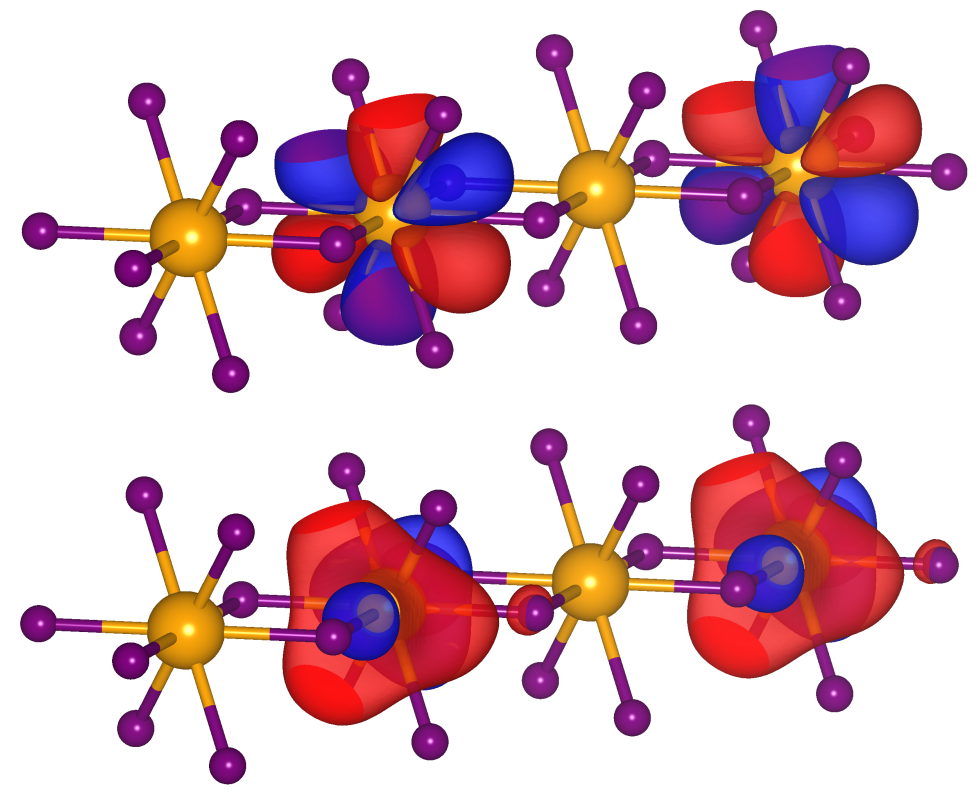

Figure 4.7: Occupied orbitals for the best candidate for $\mathrm{U}=4 \mathrm{eV} \mathrm{J}=1 \mathrm{eV}$. The Wannier function's are identical on each U site.

\subsection{Chapter Summary}

It has been demonstrated that the firefly algorithm is a powerful technique to explores multiple regions of orbital occupation PES in the d-electron and f-electron systems. Since multiple regions are simultaneously probed, a multitude stable orbital configurations can be obtained quickly. The method does not depend on specific details of the system other than the particular orbitals which are being corrected, which also means that any DFT code that can constrain the occupation matrix can be used. The efficiency of the method coupled with its independence with respect to system dependent details makes FA well-suited to the problem of locating possible stable orbital configurations.

The implementation was tested for two examples: the perovskite $\mathrm{KCoF}_{3}$ with $d$-orbitals and $\mathrm{UO}_{2}$ with $f$-electrons where in both cases the FA was able to identify a multitude of metastable states with differing occupations and to identify the lowest energy one among the series. Moreover, the method itself could be used to sweep over many values of $U$ and $J$ in order to locate the values which yield an occupancy which is in agreement with experiment. This method improves upon the currently used brute-force methods in which the ground state is found through 


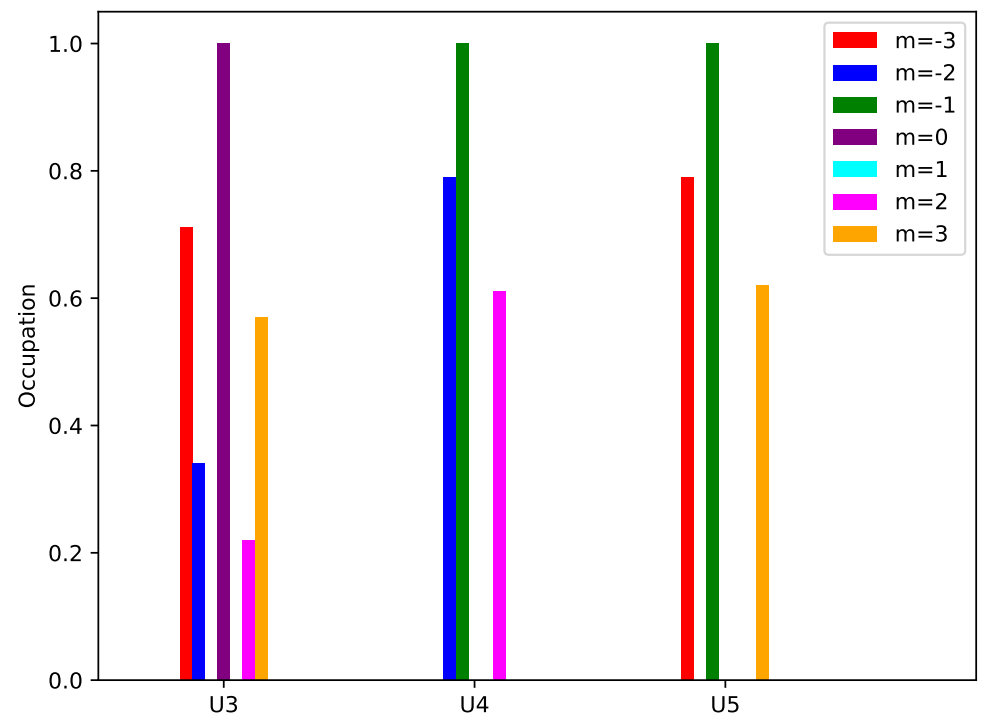

Figure 4.8: Orbital occupations ground state obtained for $\mathrm{UO}_{2}$ and $U=3,4$ and $5 \mathrm{eV}$ with $\mathrm{J}=1 \mathrm{eV}$.

changing the initial occupation matrix by hand. At the same time, it can be used in conjuction of the optimization of magnetic orientation in non collinear calculations, by interfacing this methodology with the one reported in Ref [82] The method could be also very important for hybrid functionals, which have the same problem of multiple minima in the orbital occupations. The addition of spin-orbit coupling could worsen the problem and such metaheuristic methods could be very handful to identify the lowest energy occupations of non-collinear magnets. [82]. 


\section{Chapter 5}

\section{NiTi}

Nitinol, an alloy of nearly equiatomic ratios (50-51\% nickel content) of nickel and titanium, is a well-known alloy which displays both super-elasticity and shapememory. This material has wide applications in thermal actuators, which replace more traditional solenoids, damping systems in structural engineering applications, such as intelligently reinforced concrete for bridges and buildings, and a wide-variety of biomedical applications due to the good biocompatibility of both nickel and titanium [30]. However, there are still issues with regards to the nature of the shape memory effect, in addition to engineering challenges. It is currently accepted that a martensitic transformation is the underlying mechanism for the shape-memory effect. Additionally, the ground0state martensite phase is pseudoelastic, meaning strains of up to $10 \%$ can be applied without plastic deformation. [30, 49, 74, 38, 106]. The martensite mechanism is the previously stated structural transition from a low-temperature martensitic phase to a hightemperature austenitic phase which occurs in the range of 20-50 C [94]. However, there exists fatigue which occurs only after a few thermal cycles, which is why the transition occurs over such a large range of temperatures. This results in an accumulation of residual martensite after the transformation to austenite, which reduces the performance of the shape-memory effect. While its strain-controlled performance is superior to most other known metals, in structural engineering applications NiTi is still subject to massive strains which induce fatigue failure. Thus, fully understanding the shape-memory effect in addition to filling out the compositional phase diagram is essential to better understand its properties. Additionally, very little is understood outside of the nearly equiatomic composition, so it is currently unknown if compositions other than the nearly equiatomic can support shamp-memory. In this work, the minima hopping method (MHM) 37, 
4] is used to determine the low-energy crystal structures on the convex hull for 34 different stoichoemetries with up to 10 atoms per unit cell. In this dissertation an exhaustive structural search is performed over different $\mathrm{Ni}_{x} \mathrm{Ti}_{1-x}$ stoichiometries, results are related to previously reported phases, and new phases that have not been presented in the literature will be discussed. First, results which are relevant to the martensitic transformation outside of the equiatomic composition will be discussed. This involves identifying stoichiometries which support both the monoclinc martensitic phase in addition to the cubic austenitie phase. The necessary austenite phase is the primary constraint, as only two stoichiometries other than the equiatomic case support a cubic phase. These are $\mathrm{Ni}_{2} \mathrm{Ti}$ and $\mathrm{Ni}_{5} \mathrm{Ti}$. Two new structures on the convex hull are reported, an orthorhombic phase with the composition $\mathrm{Ni}_{5} \mathrm{Ti}$, and a tetragonal phase for the composition $\mathrm{NiTi}_{2}$. Additionally, two metastable structures with interesting mechanical properties were found: A monoclinic phase for the $\mathrm{NiTi}_{2}$ which has a negative Poisson ratio (NPR) and a monoclinc phase for NiTi with negative linear compressibility (NLC).

Materials with NPR are known as auxetic materials, a term coined in the 1990's [29] which comes from the Greek $\alpha v \eta \tau \iota \kappa o \sigma$, meaning "that which tends to increase". When an auxetic material is subjected to compressive strain, the material contracts in a direction orthogonal to the applied strain, and when subjected to tensile strain, the structure expands in a direction orthogonal to the applied strain. Since their discovery, several mechanisms based on geometric models have been proposed for the realization of this unique property, but most were initially believed to be nothing more than mathematical curiosities. In 1987, with the creation of an auxetic foam based upon a re-entrant honeycomb cellular structure, this property was fully realized in a material system. Auxetics are difficult to shear, but are relatively compressible, in contrast to materials with positive Poisson ratio. As a result, they show enhanced indentation resistance, larger fracture toughness, and enhanced impact and vibrational energy absorption.[18] Most materials have Poisson ratios in the range of 0.2-0.4 [43] but, in anisotropic materials, the NPR can have values which fall far outside this range[34].

The most common examples of auxetic crystals are $\alpha-\mathrm{SiO} 2$ crystobalite, and the zeolites. NPR in crystobalite is explained by a slight variant of the rotating rigid deformation model, and zeolites follow the semi-rigid deformation model[44]. Recently, using high throughput methods, the group of K. Persson in Berkeley has been able to identify an interesting set of auxetic polycrystalline solids[24]. The search was performed within the Materials Project database[52], but only identified 7 auxetic cases among a total of around 67000 crystal structures. Monoclinic $\mathrm{SiO}_{2}$ was found to yield the largest negative value of the Poisson ratio, with a 
value of -0.6. The reported structures have a common mechanism, the rotating rigid deformation model, which explains their unusual elastic behavior. Finally, another study has shown that (seemingly defying common sense) 69\% of cubic elemental metals show NPR along the [110] direction[9]. None of the reported cubic elemental structures display auxetic behavior along axial directions.

In general, the compressibility of a material is a measure of of the collapse of the linear dimensions of the material when subjected to hydrostatic strain:

$$
K_{L}=-\frac{1}{L} \frac{d L}{d P}
$$

where $L$ is the original length along a given direction, and $\frac{d L}{d P}$ is the rate of change of the length with respect to pressure. The negative sign ensures the positive compressibilities correspond in a reduction in length when subjected to external pressure. There is a rare class of materials with the counter-intutive property of NLC, these are materials where $\frac{d L}{d P}$ is negative. From the form of this expression, the units of compressibility are inverse pressure, or $\mathrm{Pa}^{-1}$. A material with this property will expand along one direction when subjected to hydrostatic strain.

\subsection{Minima Hopping}

The challenge structural prediction attempts to meet efficiently is the systematic exploration of the potential energy surface. This is a multidimensional surface given by the free energy of the the different configurations of the degrees of freedom of any given system, which in the case of structural search are the ionic coordinates and unit cell lattice vectors. This means this is at the very least a $3 \mathrm{~N}+3$ dimensional surface for a system with N atoms. Energetically stable configurations of ions will correspond to minima on this surface, so the problem of structural prediction is equivalent to locating the minma, both local and global, of the PES. This is a formidable task in general, and there are a number of different global search methods which have been used for structural search. Some of the more popular methods are genetic algorithms[72], metaheuristic methods such as the particle swarm[71] and firefly algorithm[8], simulated annealing, and basis hopping. Here the minima hopping method [37, 4] is used to locate the lowenergy phases of binary NiTi. This method uses both density functional theory (DFT) and short molecular dynamics (MD) simulations to effectively hop around the PES. The searcher begins with a single point on the PES, which corresponds to some configuration of atoms in a box. This point will reside within the basin of 
attraction of a local minimum of the PES, and geometric relaxation is performed with DFT to reach this minimum. To escape the minimum, the initial velocities of an MD simulation is aligned along the softest-mode direction found from the local environment. Alongside the Bell-Evans-Polyani principle [88], this forces the searcher into a new basin of attraction, where the process then repeats. In addition, a feedback mechanism is implemented to avoid revisiting previously located minima.

In this work, the MHM is used to explore the PES of $\mathrm{Ni}_{x} \mathrm{Ti}_{1-x}\left(0_{j} \mathrm{x}_{i} 1\right)$ with up to ten atoms per cell. With this constraint in mind, thirty-four different stoichiometries were explored. These are $\mathrm{NiTi}, \mathrm{NiTi}_{2}, \mathrm{NiTi}_{3}, \mathrm{NiTi}_{4}, \mathrm{NiTi}_{5}, \mathrm{NiTi}_{6}$, $\mathrm{NiTi}_{7}, \mathrm{NiTi}_{8}, \mathrm{NiTi}_{9}, \mathrm{Ni}_{2} \mathrm{Ti}, \mathrm{Ni}_{2} \mathrm{Ti}_{3}, \mathrm{Ni}_{2} \mathrm{Ti}_{5}, \mathrm{Ni}_{2} \mathrm{Ti}_{7}, \mathrm{Ni}_{3} \mathrm{Ti}, \mathrm{Ni}_{3} \mathrm{Ti}_{2}, \mathrm{Ni}_{3} \mathrm{Ti}_{4}, \mathrm{Ni}_{3} \mathrm{Ti}_{5}$, $\mathrm{Ni}_{3} \mathrm{Ti}_{7}, \mathrm{Ni}_{4} \mathrm{Ti}, \mathrm{Ni}_{4} \mathrm{Ti}_{3}, \mathrm{Ni}_{4} \mathrm{Ti}_{5}, \mathrm{Ni}_{5} \mathrm{Ti}, \mathrm{Ni}_{5} \mathrm{Ti}_{2}, \mathrm{Ni}_{5} \mathrm{Ti}_{3}, \mathrm{Ni}_{5} \mathrm{Ti}_{4}, \mathrm{Ni}_{6} \mathrm{Ti}, \mathrm{Ni}_{6} \mathrm{Ti}_{2}, \mathrm{Ni}_{7} \mathrm{Ti}$, $\mathrm{Ni}_{7} \mathrm{Ti}_{2}, \mathrm{Ni}_{7} \mathrm{Ti}_{3}, \mathrm{Ni}_{8} \mathrm{Ti}$, and $\mathrm{Ni}_{9} \mathrm{Ti}$. Within each stoichiometry, differing numbers of atoms were included such that the total number of atoms never exceeded ten atoms per cell. For example, in the equiatomic stoichiometry, cells with two, four, six, eight, and ten atoms per cell were considered. However, for example, in $\mathrm{Ni}_{7} \mathrm{Ti}$, there are already eight atoms per cell, so only the singular case was considered. To generate the convex hull, for each stoichiometry and size up to forty structures were found using MHM, re-optimized, and sorted energetically. The formation energy of each structure is determined using the following expression:

$$
(1-x) E_{L}-x E_{R}
$$

where $\mathrm{x}$ is given $\frac{N_{L}}{N_{L}+N_{R}}$, the ratio of the pristine elements of each binary structure. $E_{L}$ is the energy/atom of the left pristine element and $E_{R}$ is the energy of the right pristine element. This forty structures were found across all cell sizes, meaning that forty were generated across all cell sizes considered for each stoichiometry. These energies were compared to pristine $\mathrm{Ni}$ and $\mathrm{Ti}$ to determine chemical stability. All low-energy structures on the convex hull are preserved, as well as structures with the same stoichemetries which were within $50 \mathrm{meV}$ of these lowenergy structures. Figure 5.1 shows the calculated convex-hull of the thirty-four calculated stoichiometries. Forty minima found by MHM were randomly selected for each stoichiometry and were geometrically relaxed with more stringent convergence criteria than the initial MHM simulation. Forty minima were chosen based upon the number of unique minima found with each MHM simulation for the differing stoichiometries. While it is a free parameter, MHM proceeds until fifty different minima are located. This cut off value provides a balance between computational efficiency and the accuracy of the resulting exploration of the PES, 
and has been found useful in terms of locating structures on the convex hull in previous works. []. Since the convergence criteria of MHM is rather rough, differing minima which correspond to physically equivalent structures are often found, so forty minima provides a large enough sampling to capture the unique minima found. These plot was obtained by optimizing these forty minima with a convergence criteria of $10^{-4} \mathrm{eV}$ for the energies, and $10^{-4} \mathrm{eV} / \mathrm{Angstrom}$ for the forces. A large number of structures potentially synthesizable were found with negative formation energy. Figure 5.2 displays the frequency of the types of structures found within this $50 \mathrm{meV}$ cutoff. The vast majority of structures found are monoclinic and triclinic. The only cubic structures found, other than the equiatomic case, are in the Ni-heavy region. Tetragonal structures are primarily found in the Ni-rich region, with the exception of one which is located for $\mathrm{Ni}_{4} \mathrm{Ti}$. However, there does not seem to be any trend with other higher-symmetry structures, as they appear across all stoichiometries. Four compositions were found with chemically stable structures, meaning that they lie on the convex hull. These compositions are NiTi2, Ni1Ti1, Ni3Ti1, and Ni5Ti1. The Ni5Ti structure is previously unreported, and both $\mathrm{Ni}_{1} \mathrm{Ti}_{2}$ and $\mathrm{Ni}_{3} \mathrm{Ti}_{1}$ have not been well-studied: the structures themselves are reported in both the Materials Project and OQMD, but no derived properties were found. Table 1 contains the crystallographic information for unique structures found on or near the convex hull for these compositions. All structures within $50 \mathrm{meV}$ of any structure found on the convex hull were further optimized, with a convergence criteria of $10^{-6} \mathrm{eV}$ for the energies, and $10^{-4} \mathrm{eV} / \AA ̊$ for the forces. Additionally, the figure contains the convex hulls from two popular materials databases, OQMD and the Materials Project [51, 52, 89]. The structures on the generated convex hull were relaxed with more stringent criteria than either of these databases, yielding slightly lower formation energies. In comparison, for $\mathrm{Ni}_{3} \mathrm{Ti}$, the results from MHM are in agreement with both databases, but for the other three stoichiometries new phases are found which are lower in energy. Table 5.1 displays the phases found on the convex hull to those which were reported in both databases. For $\mathrm{NiTi}_{2}$, both OQMD and the Materials Project report a low-energy $F d-3 m$ phase, while MHM located a $I 4 / \mathrm{mmm}$ phase which is lower in energy. For equiatomic NiTi,an orthorhombic phase with space group Pnma is found which is lower in energy than the Cnmm phase which is reported in the Materials Project and the $P 2{ }_{1} / m$ phase reported in OQMD. 


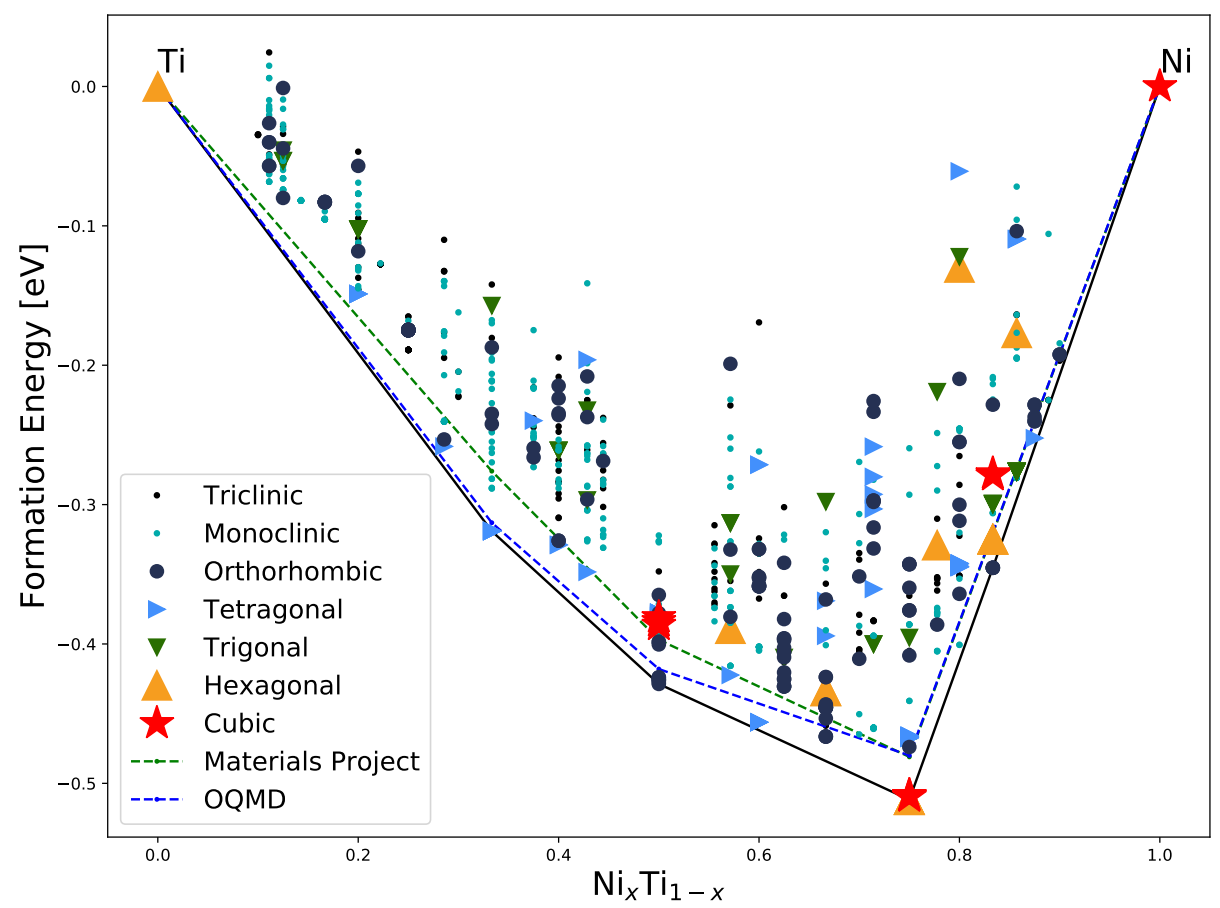

Figure 5.1: Convex hull for NiTi. Only structures found with MHM are displayed, the convex hulls generated from OQMD and Materials Project are not displayed, to reduce clutter.

\subsection{Computational Details}

All relaxations, both ionic and electric, were performed using DFT as implemented in the Vienna Ab-Initio Simulation Package (VASP) [63, 64]. VASP is used here since the MHM software was written to interface with it. The Projector Augmentation Wave (PAW) [12, 62] method was used to describe the electrons. For $\mathrm{Ni}$, sixteen valence electrons were used, and for Ti ten. The generalized gradient approximation (GGA) as parameterized by Perdew-Burke-Ernzerhof (PBE) was used for the exchange-correlation functional [85]. A Monkhorst-Pack kmesh, of varying size based on the size of the cell considered, was used to sample the Brillouin Zone. For hexagonal structures,a gamma-centered mesh was enforced to correctly capture hexagonal symmetry. The k-mesh was optimized to ensure convergence of less than $10^{-6}$ in total energy, and all forces were con- 


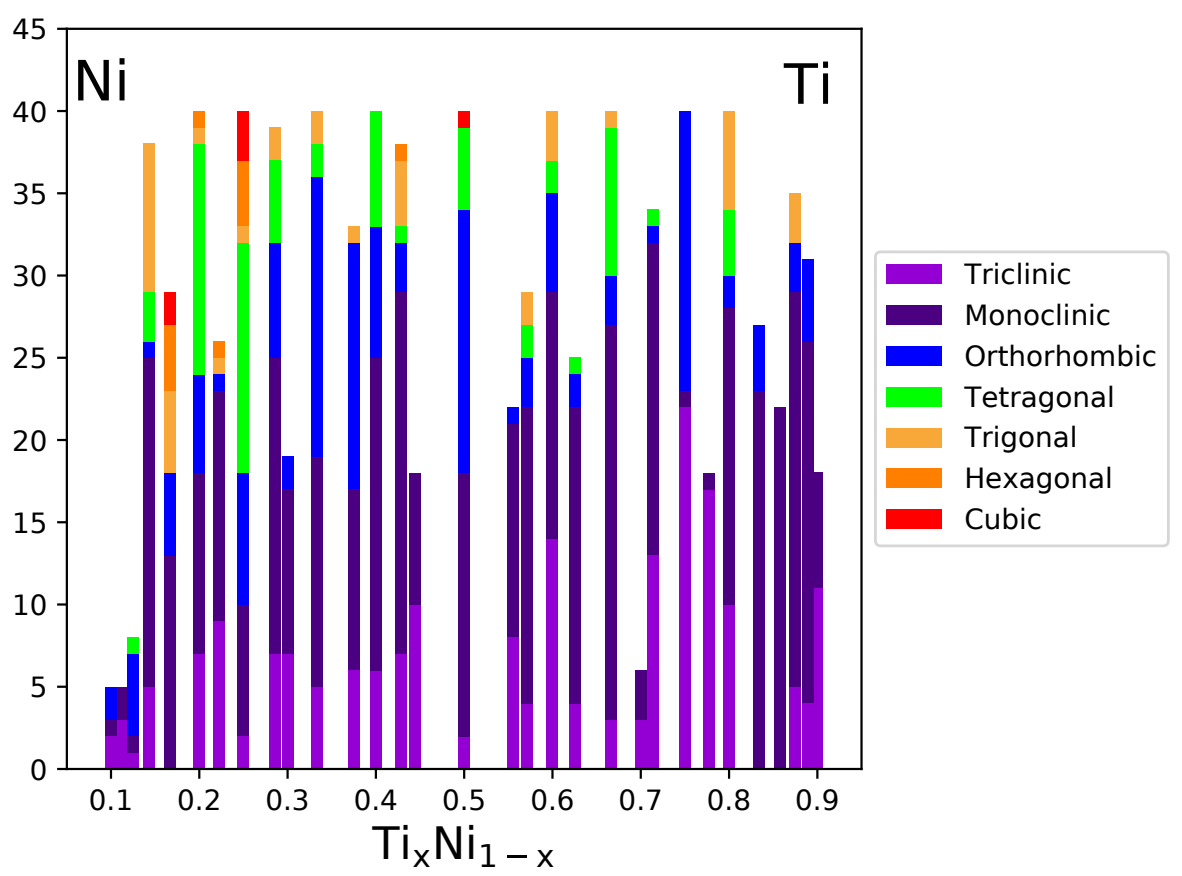

Figure 5.2: Frequency of each space group for all considered stoichiometries.

verged to better than $10^{-4}$ ev/Angstrom. Electronic energies were converged to within $10^{-6} \mathrm{eV}$ and strain was converged to less than $10^{-3} \mathrm{kBarr}$ in the self consistent cycle. All DFT calculations were performed at both zero pressure and temperature.

Thermal and mechanical stability were assessed, and mechanical properties were found by determining elastic constants through the use of lattice distortions to derive the stress-strain relationship in order to construct the elastic tensor, as implemented in the VASP code [102]. The frozen-phonon method, as implemented in the PHONOPY software [97], was used to assess thermal stability. Phonon calculations were performed by constructing supercells to generate phonon spectra. Supercells were constructed of varying size such that the resulting supercell is as close to cubic as possible. Elastic constants were found through calculating the elastic tensor by deriving the stress-strain relationship by performing six finite distortions for each lattice, as implemented in VASP. Using the elastic constants, mechanical stability was assessed and mechanical properties, such as the Bulk, 
Young's, and Shear moduli, as well as the Poisson ratio, were found. The Young's modulus is defined as the ratio of applied uniaxial stress to the strain, $E=\frac{\sigma}{\epsilon}$. The bulk modulus is defined by the expression $K=P \frac{d P}{d V}$, which is the product of pressure with the derivative of the pressure with respect to volume. This shows how resistant a material is to compression. The shear modulus is defined by the ratio of the shear stress to the shear strain, $G=\frac{F / A}{\delta X / L}$. The units for each are pascals The Poisson ratio is a unitless measure of the transverse response of a material subjected to uniaxial strain, $\gamma=\frac{\epsilon_{\text {trans }}}{\epsilon_{a x i a l}}$. The angular dependence of these properties was determined using the ELATE open-source software package [32]. Thermal properties were calculated through the use of the quasi-harmonic Debye model as implemented in the gibbs 2 code [79].

\subsection{Results And Discussion}

The results for each composition will now be discussed in detail. For each composition, results will be compared to previous work. From there, the properties of new structures will be expounded upon and compared to previously known structures.

First, a few properties will be discussed for the collective convex hull structures. the specific heat will be discussed for the convex hull structures. Figure 5.4 shows the $C_{v} / T^{3}$ curve for the different structures found using MHM. This plot displays three regimes: the first corresponds to the contribution from acoustic phonon modes which leads to a $T^{3}$ behavior at very low temperatures. In the second regim, optical phonon modes dominate, which are characterized by an Einstein isotropic peak around $150 \mathrm{~K}$. In third regime all modes contribute to $C_{v}$ so $C_{v} / T^{3}$ decays with a $T^{3}$ power-law. The analysis of $C_{v}$ neglects the contribution from the conduction electrons. In this plot, the Einstein peak is larger for the $\mathrm{NiTi}_{2}$ phase by almost twice in comparison to $\mathrm{Ni}_{3} \mathrm{Ti}$ and $\mathrm{Ni}_{5} \mathrm{Ti}$. This observation can be correlated to the phonon spectra. The optical modes of $\mathrm{NiTi}_{2}$ are significantly higher in frequency that the other phases, the optical modes span a range of 50-150 THz. The optical modes of $\mathrm{Ni}_{3} \mathrm{Ti}, \mathrm{NiTi}$ and $\mathrm{Ni}_{5} \mathrm{Ti}$ have values in the ranges of 4.5-9 THz, $0.12-0.27 \mathrm{THz}$, and $0.12-0.24 \mathrm{THz}$, respectively. This ordering is equivalent to the ordering of the peaks of the $C_{v} / T^{3}$ curve.

Secondly, variation of elastic properties with respect to composition is plotted in Figure 5.3. The most striking feature of this plot is the anomalous decrease in the bulk modulus, Young's modulus, and shear modulus of orthrhombic NiTi. The second is that the bulk modulus and shear modulus of both $\mathrm{Ni}_{3} \mathrm{Ti}$ and $\mathrm{Ni}_{5}$ 


\begin{tabular}{|c|c|c|c|c|c|c|c|c|c|}
\hline Composition & $\begin{array}{l}\text { Atoms per } \\
\text { unit cell }\end{array}$ & Space Group & $\mathrm{a}(\AA)$ & $\mathrm{b}(\AA)$ & c $(\AA)$ & $\alpha$ & $\beta$ & $\gamma$ & Energy/atom (eV/atom) \\
\hline $\mathrm{NiTi}_{2}$ & 6 & $\mathrm{I} 4 / \mathrm{mmm}$ & 5.30 & 5.30 & 5.30 & 147.0 & 147.0 & 47.3 & -7.49 \\
\hline $\mathrm{NiTi}_{2}[52$ & 24 & Fd-3m & 7.97 & 7.97 & 7.97 & 60.0 & 60.0 & 60.0 & -7.467 \\
\hline $\mathrm{NiTi}_{2}$ & 24 & $\mathrm{Fd}-3 \mathrm{~m}$ & 7.97 & 7.97 & 7.97 & 60.0 & 60.0 & 60.0 & -7.465 \\
\hline $\mathrm{NiTi}_{2} 89$ & 24 & $\mathrm{Fd}-3 \mathrm{~m}$ & 7.95 & 7.95 & 7.95 & 60.0 & 60.0 & 60.0 & \\
\hline $\mathrm{NiTi}_{2}[77$ & 24 & Fd-3m & 7.95 & 7.95 & 7.95 & 60.0 & 60.0 & 60.0 & \\
\hline $\mathrm{NiTi}_{2}$ & 24 & $\mathrm{Fd}-3 \mathrm{~m}$ & 7.97 & 7.97 & 7.97 & 60.0 & 60.0 & 60.0 & \\
\hline NiTi & 4 & $\mathrm{P} 2_{1} / \mathrm{m}$ & 2.92 & 4.01 & 4.89 & 90.0 & 106.3 & 90.0 & -7.235 \\
\hline NiTi & 4 & $\mathrm{P} 2_{1} / \mathrm{m}$ & 2.93 & 4.06 & 4.75 & 90.0 & 100.9 & 90.0 & -7.235 \\
\hline $\mathrm{NiTi} 89$ & 4 & $\mathrm{P} 2{ }_{1} / \mathrm{m}$ & 2.91 & 4.03 & 4.70 & 90.0 & 98.4 & 90.0 & -7.050 \\
\hline NiTi $\overline{\overline{89}}$ & 4 & $\mathrm{P} 2_{1} / \mathrm{m}$ & 2.84 & 4.13 & 4.61 & 90.0 & 95.5 & 90.0 & -7.044 \\
\hline NiTi $\overline{\overline{89}}$ & 4 & $\mathrm{P} 2_{1} / \mathrm{m}$ & 2.75 & 4.22 & 4.63 & 90.0 & 92.1 & 90.0 & -7.041 \\
\hline NiTi $\mid \overline{\overline{66}}$ & 4 & $\mathrm{P} 2{ }_{1} / \mathrm{m}$ & 2.90 & 4.11 & 4.65 & 90.0 & 97.8 & 90.0 & \\
\hline NiTi & 4 & $\mathrm{Cmcm}$ & 4.91 & 4.91 & 4.01 & 90.0 & 90.0 & 145.2 & -7.234 \\
\hline NiTi[52. & 4 & $\mathrm{Cmcm}$ & 4.92 & 4.92 & 4.02 & 90.0 & 90.0 & 145.5 & -7.236 \\
\hline NiTi & 8 & Pnma & 5.20 & 5.20 & 4.20 & 90.0 & 90.0 & 90.0 & -7.236 \\
\hline $\mathrm{Ni}_{3} \mathrm{Ti}$ & 8 & $\mathrm{P}_{3} / \mathrm{mmc}$ & 5.11 & 5.11 & 4.17 & 90.0 & 90.0 & 120.0 & -6.777 \\
\hline $\mathrm{Ni}_{3} \mathrm{Ti}$ & 16 & $\mathrm{P}_{3} / \mathrm{mmc}$ & 5.10 & 5.10 & 8.31 & 90.0 & 90.0 & 120 & -6.791 \\
\hline $\mathrm{Ni}_{3} \mathrm{Ti}$ & 8 & $\mathrm{P}_{3} / \mathrm{mmc}$ & 5.10 & 5.10 & 4.15 & 90.0 & 90.0 & 120.0 & -6.581 \\
\hline $\mathrm{Ni}_{3} \mathrm{Ti}[89$ & 16 & $\mathrm{P}_{3} / \mathrm{mmc}$ & 5.08 & 5.08 & 8.29 & 90.0 & 90.0 & 120.0 & -6.594 \\
\hline $\mathrm{Ni}_{3} \mathrm{Ti}$ & 4 & $\mathrm{Pm}-3 \mathrm{~m}$ & 3.61 & 3.61 & 3.61 & 90.0 & 90.0 & 90.0 & -6.774 \\
\hline $\mathrm{Ni}_{3} \mathrm{Ti} / 52$ & 4 & $\mathrm{Pm}-3 \mathrm{~m}$ & 3.94 & 3.94 & 3.94 & 90.0 & 90.0 & 90.0 & -7.448 \\
\hline $\mathrm{Ni}_{3} \mathrm{Ti}$ & 4 & $\mathrm{Pm}-3 \mathrm{~m}$ & 3.60 & 3.60 & 3.60 & 90.0 & 90.0 & 90.0 & -6.539 \\
\hline $\mathrm{Ni}_{5} \mathrm{Ti}$ & 6 & $\mathrm{Cmmm}$ & 3.57 & 3.60 & 5.63 & 90.0 & 90.0 & 90.0 & -6.428 \\
\hline
\end{tabular}

Table 5.1: Crystallographic information for structures found on the convex hull with MHM. In addition, low-energy structures from both OQMD[89], Materials Project[51], and experiment are listed in the table. 


\begin{tabular}{|c|c|c|c|c|c|c|c|c|c|c|c|c|}
\hline Composition & $\begin{array}{l}\text { Space } \\
\text { Group }\end{array}$ & B (GPa) & $\mathrm{E}(\mathrm{GPa})$ & $\mathrm{S}(\mathrm{GPa})$ & $\nu(\mathrm{GPa})$ & $\mathrm{B} / \mathrm{G}$ & $\mathrm{v}_{l}(\mathrm{~m} / \mathrm{s})$ & $\mathrm{v}_{t}(\mathrm{~m} / \mathrm{s})$ & $\mathrm{v}_{a v g}(\mathrm{~m} / \mathrm{s})$ & $\begin{array}{l}\text { P-wave } \\
\text { modulus }\end{array}$ & $\begin{array}{c}\text { Universal } \\
\text { anisotropy }\end{array}$ & $\begin{array}{c}\text { Log-Euclidean } \\
\text { anisotropy }\end{array}$ \\
\hline $\mathrm{Ti}$ & $\mathrm{P} 6 / \mathrm{mmm}$ & 113.5 & 157.0 & 61.9 & 0.2 & 1.8 & 6495 & 3649 & 4060 & 196.01 & 0.232 & 0.234 \\
\hline $\mathrm{NiTi}_{2}$ & $\mathrm{I} 4 / \mathrm{mmm}$ & 145.2 & 170.2 & 65.2 & 0.3 & 2.2 & 6307 & 3343 & 3736 & 232.22 & 0.421 & 0.416 \\
\hline $\mathrm{NiTi}_{2}$ & $\mathrm{Fd}-3 \mathrm{~m}$ & 143.0 & 35.9 & 98.0 & 0.361 & 3.97 & 4866 & 2482 & 2781 & 191.00 & 2.808 & 2.296 \\
\hline $\mathrm{NiTi}_{2} 98$ & $\mathrm{Fd}-3 \mathrm{~m}$ & 146.1 & 44.4 & 49.4 & 0.348 & 2.96 & & & & & & \\
\hline $\mathrm{NiTi}_{2} 52$ & Fd-3m & 141.7 & 99.1 & 35.8 & 0.383 & 3.96 & & & & & & \\
\hline NiT1 & Pnma & 117.1 & 128.1 & 48.6 & 0.3 & 2.4 & 5296 & 2738 & 3066 & 181.92 & 2.476 & 2.072 \\
\hline $\mathrm{NiTi}$ & $\mathrm{P} 2_{1} / \mathrm{m}$ & 158.0 & 111.1 & 40.8 & 0.4 & 3.9 & 5751 & 2521 & 2846 & 212.45 & 8.350 & 5.057 \\
\hline $\mathrm{Ni}_{3} \mathrm{Ti}$ & $\mathrm{P}_{3} / \mathrm{mmc}$ & 196.1 & 233.2 & 89.6 & 0.3 & 2.2 & 6323 & 3369 & 3764 & 315.53 & 0.299 & 0.299 \\
\hline $\mathrm{Ni}_{5} \mathrm{Ti}$ & $\mathrm{Cmmm}$ & 199.2 & 230.6 & 88.3 & 0.3 & 2.3 & 6199 & 3271 & 3657 & 316.90 & 0.886 & 0.840 \\
\hline $\mathrm{Ni}$ & Fm-3m & 207.2 & 211.9 & 80.0 & 0.3 & 2.6 & 5901 & 2976 & 3338 & 313.67 & 1.418 & 1.286 \\
\hline $\mathrm{W}[51]$ & $\mathrm{Im}-3 \mathrm{~m}$ & 304.0 & 380.5 & 147.5 & 0.3 & 2.06 & & & & & & \\
\hline Stainless Steel 11 & $\mathrm{fcc}$ & 152 & 198 & 77 & 0.28 & 1.98 & & & & & & \\
\hline Stainless Steel $\overline{90}$ & $\mathrm{fcc}$ & $142-186$ & $195-208$ & $75-82$ & $0.27-0.32$ & $1.88-2.45$ & & & & & & \\
\hline $\mathrm{NiTi}_{2}$ & $\mathrm{Cl}_{2} / \mathrm{m}$ & 140.9 & 75.7 & 27.0 & 0.4 & 5.22 & 5556 & 2170 & 2460 & 176.91 & 3.993 & 3.023 \\
\hline NiTi & $\mathrm{P} 2 / \mathrm{m}$ & 155.1 & 97.9 & 35.1 & 0.4 & 4.41 & 5447 & 2166 & 2454 & 193.49 & 3.876 & 2.956 \\
\hline
\end{tabular}

Table 5.2: Elastic properties for convex hull structures found with MHM. The values are the averages of the Voigt and Reuss methods of determining the values of each elastic property from the elastic constants. Other strong materials are included for comparison

are enhanced in comparison to the pristine elements. Since $\mathrm{Ni}_{5} \mathrm{Ti}$ is previously unreported, this enhancement will be discussed in greater detail.

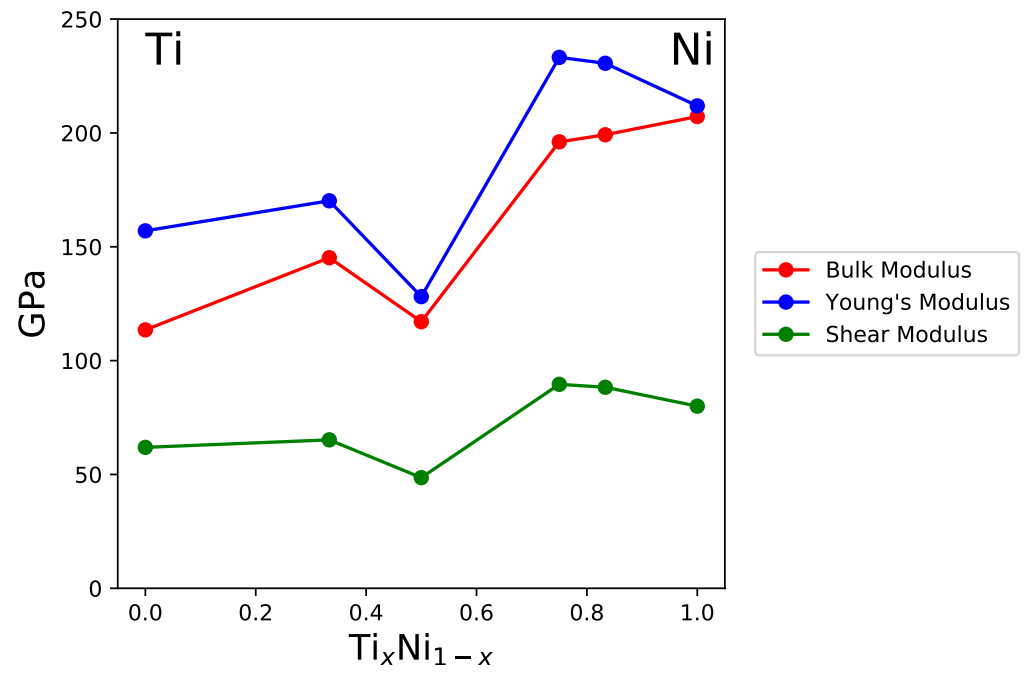

Figure 5.3: Varation of elastic properties with respect to composition for the structures on the convex hull. 


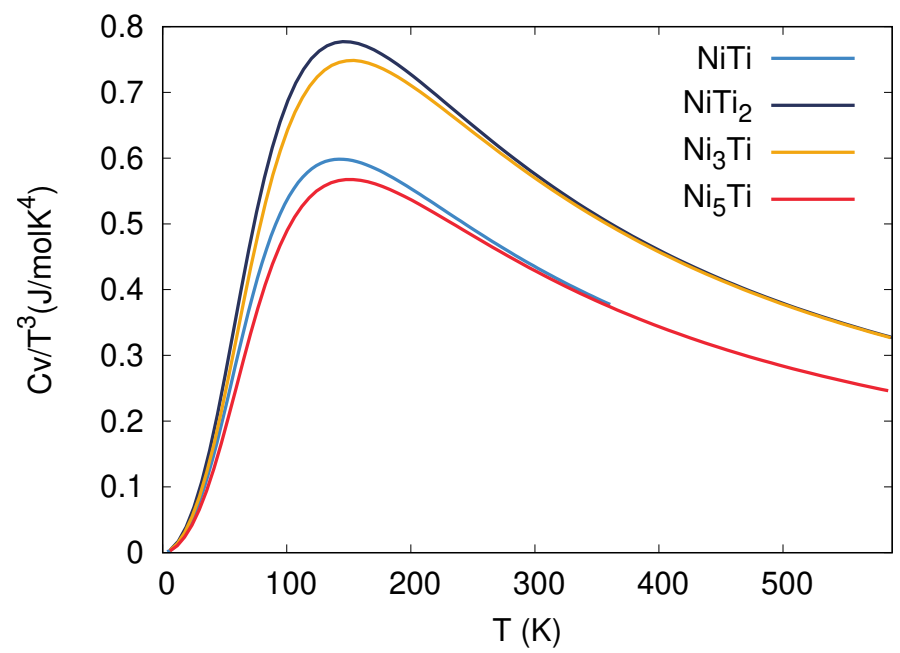

Figure 5.4: $\mathrm{C}_{v} / \mathrm{T}^{3}$ for structures on the convex hull. The primary contribution at Einstein peaks around $150 \mathrm{~K}$ are from the optical phonon modes. The ordering of the value of these peaks respects this, as $\mathrm{NiTi}_{2}$ has the highest frequency optical modes, and Ni5Ti has the lowest frequency phonon modes.

\section{$5.4 \mathrm{NiTi}_{2}$}

In both the OQMD and Materials Project databases, the low-energy phase on the convex hull is a structure with space group $F d-3 m$, yet a different structure is found convex hull, with space group $14 / \mathrm{mmm}$ which is lower in energy than this previously reported phase. However, while the $14 / \mathrm{mmm}$ has not been experimentally realized, the $F d-3 m$ structure has been experimentally synthesized [104]. Before any comparisons are drawn between the two, it should be noted that a high-energy cubic phase is necessary for the martensitic transformation which drives the shapememory effect in near equiatomic NiTi. While it is slightly higher in energy than the low-energy that was found using MHM, since the cubic phase of $\mathrm{NiTi}_{2}$ has been previously reported as the ground state, it is unlikely that $\mathrm{NiTi}_{2}$ supports the shape-memory effect. The structural parameters for this phase are listed in Table 5.12. The conventional cell is also displayed in this table.

However, in both databases structures there are 24 atoms in the primitive cell for $F d-3 m$, which puts them outside the search space for the calculations performed with MHM, as only cells of up to ten atoms where considered. Standalone calculations were performed for the $F d-3 m$ structure, with initial structural information from the Materials Project. This geometry of this structure was opti- 
mized based on the same criterion as described in the computational details section. From this optimization, the new $14 / \mathrm{mmm}$ structure on the convex hull is 22.8 meV/atom lower in energy than the cubic phase. Additionally, the elastic constants for both structures are listed in Table 5.2 In both cases, the elastic constants satisfied the Born conditions, which indicates that both are mechanically stable. The bulk moduli for both structures are roughly equivalent, but the shear-modulus and Young's modulus are enhanced in the low-energy tetragonal structure. The band structure of the $F d-3 m$ phase is displayed in Figure 5.7

The phonon spectra for the $14 / \mathrm{mmm}$ structure is displayed in Figure 5.5. All phonon modes are positive, idincating dynamical stability.

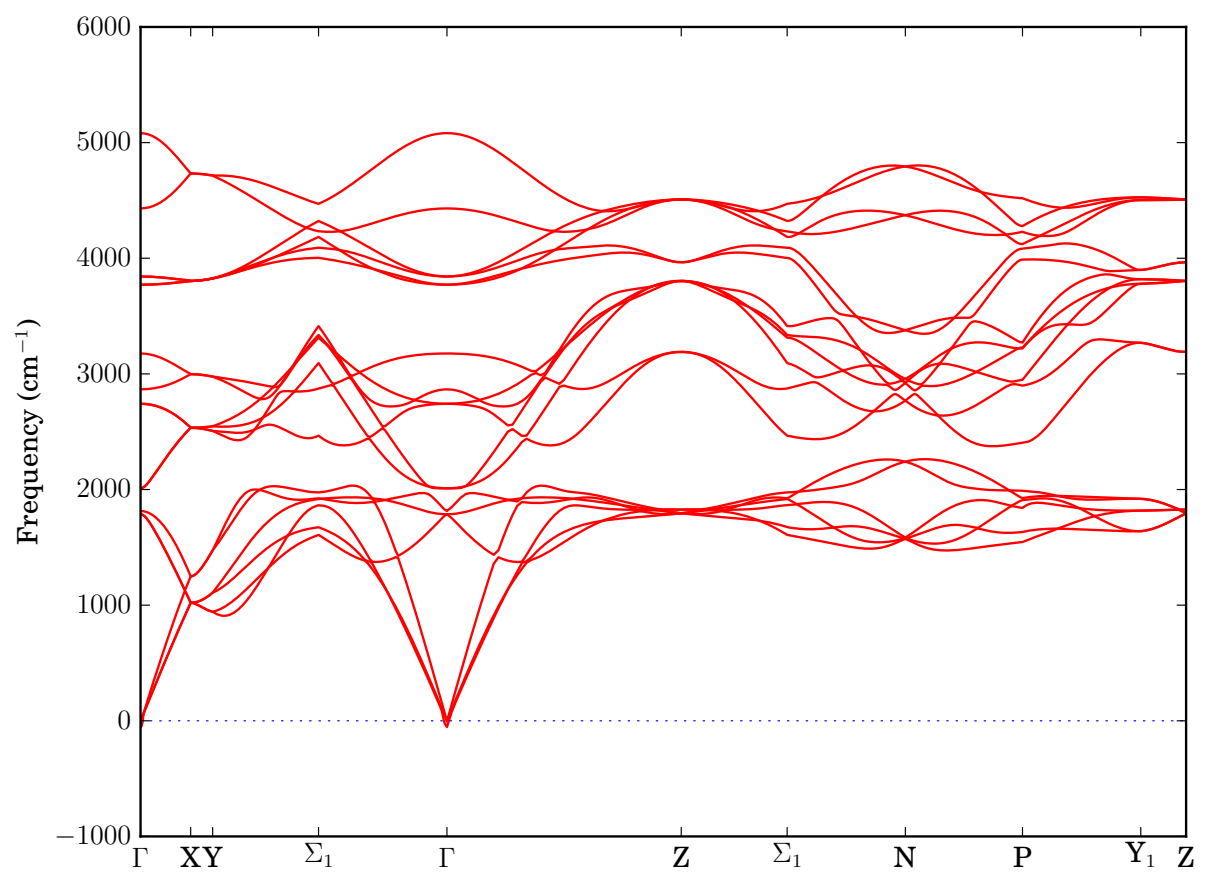

Figure 5.5: Phonon spectra for $\mathrm{NiTi}_{2}$ with space group $\mathrm{I} / \mathrm{mmm}$

The calculated electronic density of states for $\mathrm{NiTi}_{2}$ with space group $14 / \mathrm{mmm}$ is presented in Figure 5.15. The density of states is non-zero at the Fermi level, so $\mathrm{NiTi}_{2}$ is metallic. In addition, the calculated densities are the same for both spin channels, indicating that the system is non-magnetic. Figure 5.6 displays the 
band structure for this phase. Because of the band flattening around the Fermi level, the Fermi surface is of interest. The Fermi surfaces of both phases are displayed in Figure 5.9a. While the Fermi surface of the cubic phase is highly connected, which is typical amongst transition metals, the Fermi surface of the tetragonal phase is far more interesting. The crystal structure of the tetragonal phase is elongated along the [001] direction, which entails that the corresponding direction in the Brillouin zone will be flat. The flatness of the Brillouin zone along this direction means the system resembles a $2 \mathrm{D}$ or layered-like system. The Fermi surface is comprised of nested corrugated cylinders, which is indicative of this behavior.

The total density of states for both phases is displayed in Figure 5.10. In both cases, the density of states for both spin-channels are identical, so the sum of both spin channels is displayed in this figure. In the new I4/ $\mathrm{mmm}$ phase, the main contribution to the DOS both below and above the Fermi level comes from Ni $d$ electrons. At the Fermi level, it can be seen that the Ni and Ti $d$ states hybridize. In the cubic case, the Ti $d$ states dominate above the Fermi level, and the $\mathrm{Ni} d$ states are the main contribution below the Fermi level.

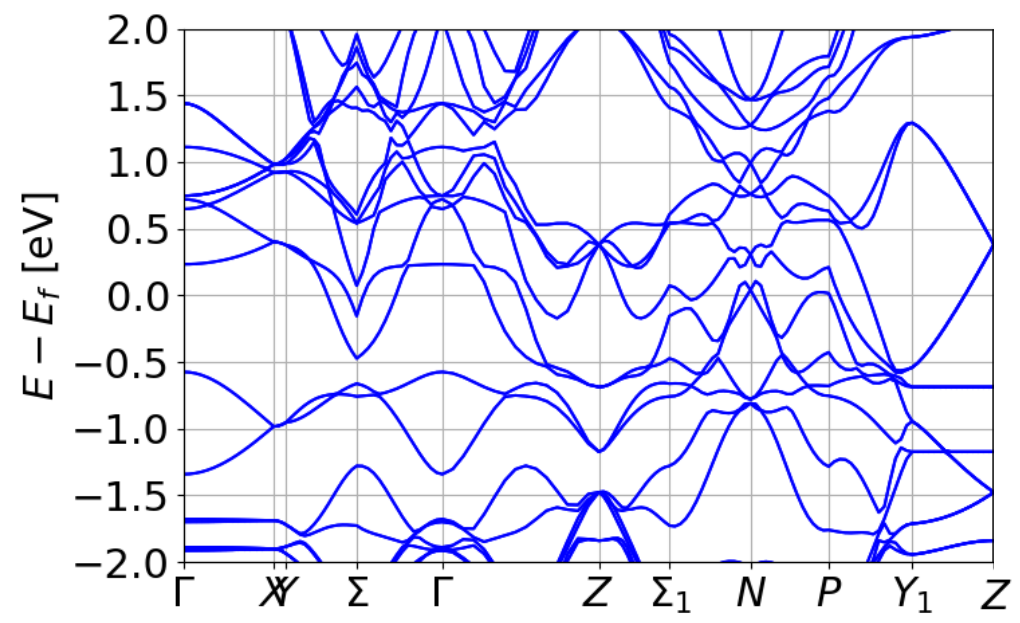

Figure 5.6: Band structure for $\mathrm{NiTi}_{2}$ with space group $\mathrm{I} / \mathrm{mmm}$. There is band flattening just above the Fermi level at the $\Gamma$ point.

An auxetic structure was found for the $\mathrm{NiTi}_{2}$ composition with space group $\mathrm{Cl}_{2} / \mathrm{m}$. The structure has inversion symmetry, and a two-fold rotation axis. The structural information for this phase is displayed in Figure 5.22. The structure has six total atoms in the unit cell. The ELATE software package [33] was used to de- 


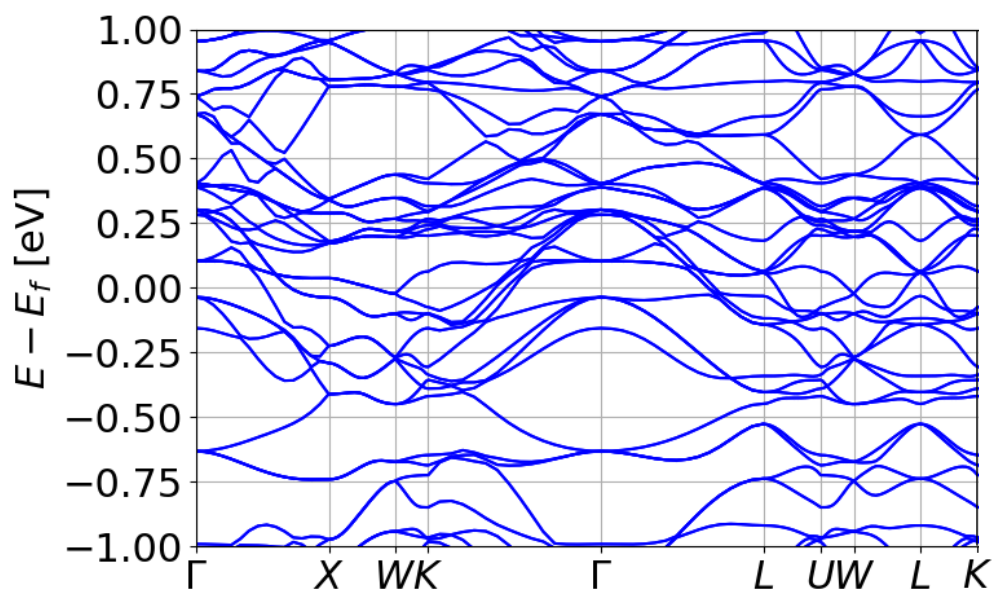

Figure 5.7: Band structure for $\mathrm{NiTi}_{2}$ with space group $\mathrm{Fd}-3 m$

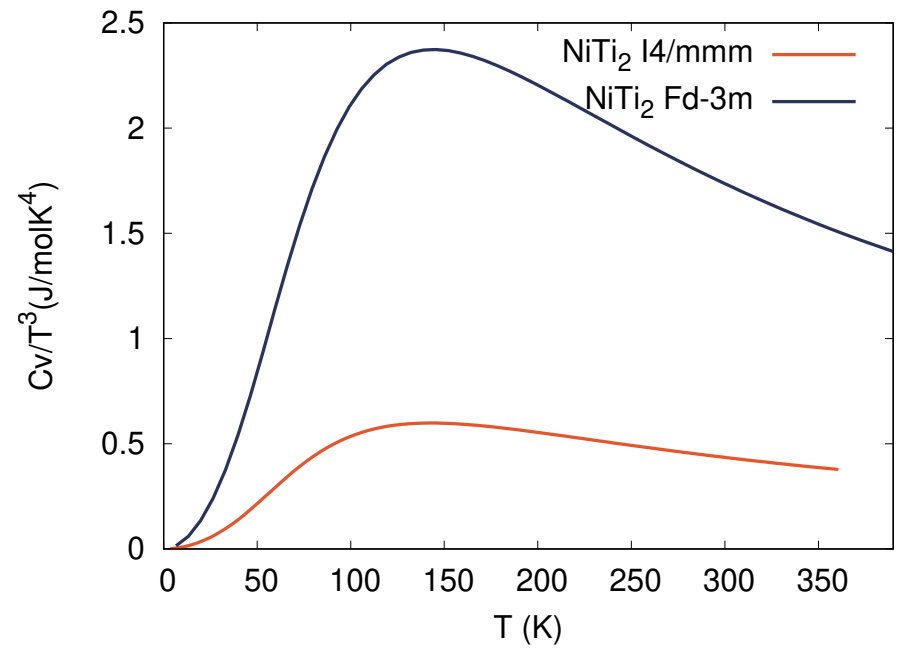

Figure 5.8: $\mathrm{C}_{v} / \mathrm{T}^{3}$ for both the low-energy structure found with MHM (space group $I 4 / \mathrm{mmm}$ ) and the previously reported low-energy structure (space group $\mathrm{Fd}-3 \mathrm{~m}$ ).

termine the angular dependence of elastic properties of $\mathrm{NiTi}_{2}$, displayed in Figure 5.18. Since two vectors are necessary to define NPR, this can be parameterized using three angles. The blue curve is along the countour of maximal angular value. The red curves displays where the Poisson ratio is negative and the green curves where it is positive. The structure attains a maximum negative Poisson ratio value of -0.31 directed along the unit vector $(0.75,0.45,-0.49)$. To show that these quan- 

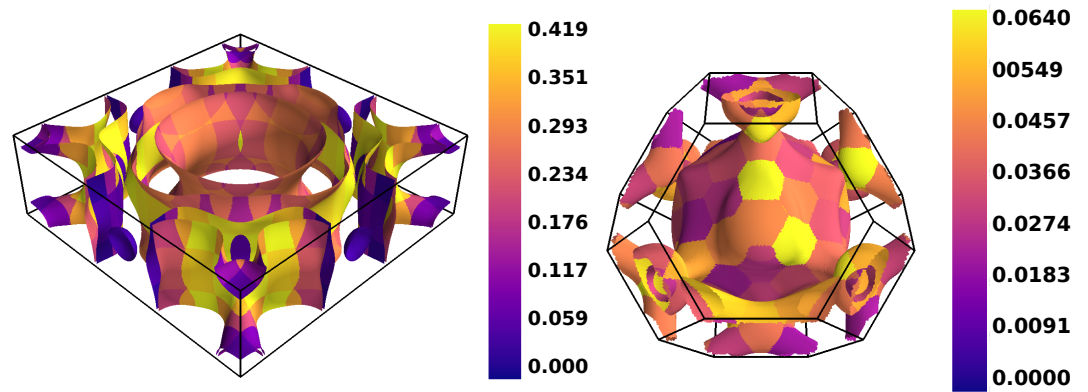

(a) Fermi surface of $\mathrm{NiTi}_{2}$ with space group $14 / \mathrm{mmm}$.

(b) Fermi surface for $\mathrm{NiTi}_{2}$ with space group $F d-3 m$.

Figure 5.9: Fermi surfaces for the two low-energy phases found for $\mathrm{NiTi}_{2}$. The scale displays the amount of the contribution to the Fermi surface from the $d$ electron states, which are the primary contribution to the density of states at the Fermi level.

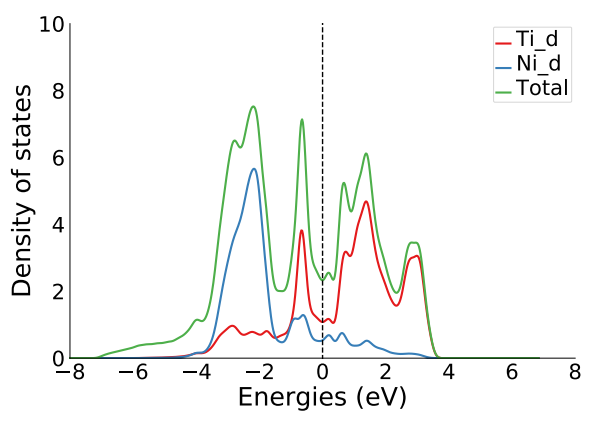

(a) Space group $14 / \mathrm{mmm}$.

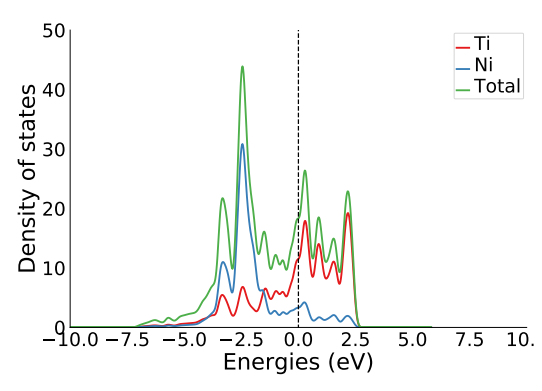

(b) Space group $F d-3 m$.

Figure 5.10: Density of states for the two low-energy phases found for $\mathrm{NiTi}_{2}$.

tities are well-converged, the convergence of the elastic constants is displayed in Figure 5.23. A k-point mesh of increasing density was used for each calculation, and the particular number of k-points is determined from the symmetry of each structure. Constrained relaxation under both tensile and compressive strain was performed to determine lattice constants and inter-atomic distances. Strain was applied by scaling the unit cell vectors along the direction of NPR in a range from $-10 \%$ to $10 \%$. Both tensile and compressive strain were applied along the unitvector where the Poisson ratio attains its maximum value. Figure 5.16 displays the relative change in the lattice parameters under applied strain. Both the a-axis and c-axis decrease when compressive strain is applied, and both increase when 


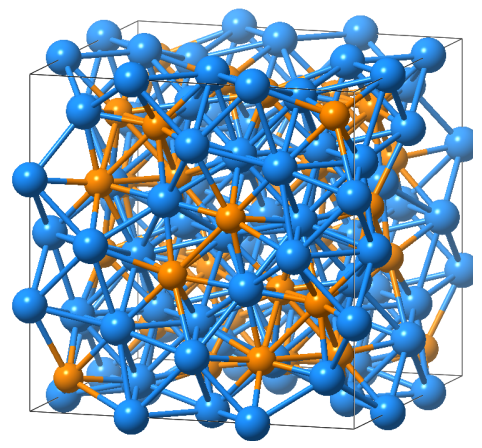

Figure 5.11: Unit cell of $\mathrm{NiTi}_{2}$ with space group $F d-3 m$

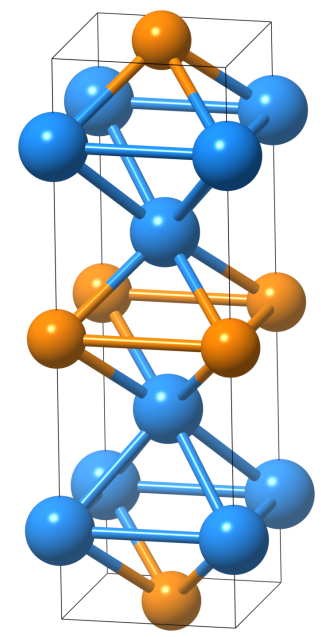

Figure 5.13: Unit cell of $\mathrm{NiTi}_{2}$ with space group $14 / \mathrm{mmm}$.

\begin{tabular}{|c|c|c|}
\hline \multicolumn{3}{|c|}{$\mathrm{NiTi}_{2} \mathrm{I} / \mathrm{mmm}$} \\
\hline $\mathbf{a}(\stackrel{\AA}{A})$ & $\mathbf{b}(\stackrel{\AA}{A})$ & c $(\stackrel{\circ}{A})$ \\
\hline 7.97 & 7.97 & 7.97 \\
\hline
\end{tabular}

\begin{tabular}{|c|c|c|c|c|c|c|c|c|c|}
\hline Atom & $\begin{array}{c}\text { Wyckoff } \\
\text { Position }\end{array}$ & $\mathrm{x}$ & $\mathrm{y}$ & $\mathrm{z}$ & Atom & $\begin{array}{c}\text { Wyckoff } \\
\text { Position }\end{array}$ & $\mathrm{x}$ & $\mathrm{y}$ & $\mathrm{z}$ \\
\hline Ni1 & $\mathrm{e}$ & 0.34 & 0.98 & 0.34 & Ti5 & $\mathrm{d}$ & 0.56 & 0.93 & 0.94 \\
Ni2 & $\mathrm{e}$ & 0.34 & 0.34 & 0.98 & Ti6 & $\mathrm{f}$ & 0.93 & 0.56 & 0.56 \\
Ni3 & $\mathrm{e}$ & 0.34 & 0.34 & 0.34 & Ti7 & $\mathrm{f}$ & 0.56 & 0.93 & 0.56 \\
Ni4 & $\mathrm{e}$ & 0.98 & 0.34 & 0.34 & Ti8 & $\mathrm{f}$ & 0.93 & 0.56 & 0.94 \\
Ni5 & $\mathrm{e}$ & 0.91 & 0.26 & 0.91 & Ti9 & $\mathrm{f}$ & 0.56 & 0.56 & 0.94 \\
Ni6 & $\mathrm{e}$ & 0.91 & 0.91 & 0.26 & Ti10 & $\mathrm{f}$ & 0.93 & 0.93 & 0.56 \\
Ni7 & $\mathrm{e}$ & 0.91 & 0.91 & 0.91 & Ti11 & $\mathrm{f}$ & 0.69 & 0.31 & 0.31 \\
Ni8 & $\mathrm{e}$ & 0.26 & 0.91 & 0.91 & Ti12 & $\mathrm{f}$ & 0.31 & 0.69 & 0.69 \\
Ti1 & $\mathrm{e}$ & 0.13 & 0.63 & 0.13 & Ti13 & $\mathrm{f}$ & 0.69 & 0.31 & 0.69 \\
Ti2 & $\mathrm{d}$ & 0.13 & 0.13 & 0.63 & Ti14 & $\mathrm{f}$ & 0.31 & 0.69 & 0.31 \\
Ti3 & $\mathrm{d}$ & 0.13 & 0.13 & 0.13 & Ti15 & $\mathrm{f}$ & 0.69 & 0.69 & 0.31 \\
Ti4 & $\mathrm{d}$ & 0.63 & 0.13 & 0.13 & Ti16 & $\mathrm{f}$ & 0.31 & 0.31 & 0.69 \\
\hline
\end{tabular}

Figure 5.12: Structural information for $\mathrm{NiTi}_{2}$ with space group $\mathrm{I} 4 / \mathrm{mmm}$.

\begin{tabular}{|c|c|c|}
\multicolumn{3}{|c}{$\mathbf{N i T i}_{2}$ I4/mmm } \\
\hline $\mathbf{a}(\stackrel{\circ}{A})$ & $\mathbf{b}(\AA)$ & $\mathbf{c}(\AA)$ \\
\hline 3.01 & 3.01 & 9.71 \\
\hline
\end{tabular}

\begin{tabular}{|c|c|c|c|c|}
\hline Atom & $\begin{array}{c}\text { Wyckoff } \\
\text { Position }\end{array}$ & $\mathrm{x}$ & $\mathrm{y}$ & $\mathrm{z}$ \\
\hline $\mathrm{Ni} 1$ & $\mathrm{~b}$ & 0.50 & 0.50 & 0.00 \\
$\mathrm{Ni} 2$ & $\mathrm{~b}$ & 0.00 & 0.00 & 0.50 \\
$\mathrm{Ti} 1$ & $\mathrm{e}$ & 0.00 & 0.00 & 0.84 \\
$\mathrm{Ti} 2$ & $\mathrm{e}$ & 0.00 & 0.00 & 0.16 \\
$\mathrm{Ti} 3$ & $\mathrm{e}$ & 0.50 & 0.50 & 0.34 \\
$\mathrm{Ti} 4$ & $\mathrm{e}$ & 0.50 & 0.50 & 0.64 \\
\hline
\end{tabular}

Figure 5.14: Structural information for $\mathrm{NiTi}_{2}$ with space group $\mathrm{I} / \mathrm{mmm}$.

tensile strain is applied. This is precisely the response expected from an auxetic material. Figure 5.17 displays the change in bonding when strain is applied. In all three subfigures, a bond is displayed if the distance between two atoms is less than $2.65 \AA$.

The density of states for this phase is displayed in Figure 5.19. The Ti $d$ electrons are the primary contribution to the density of states at the Fermi level. In addition they are the primary contribution just below the Fermi level $(>-2 \mathrm{eV})$ 


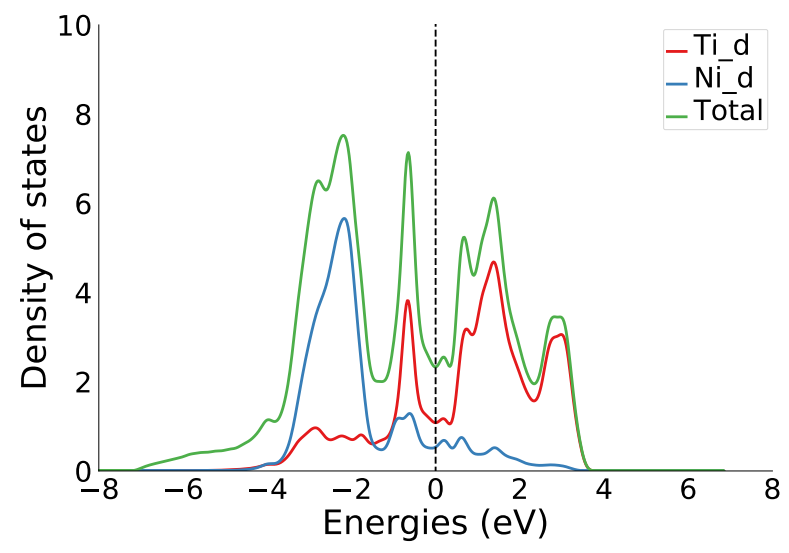

Figure 5.15: Density of states for $\mathrm{NiTi}_{2}$ with space group $\mathrm{I} / \mathrm{mmm}$.

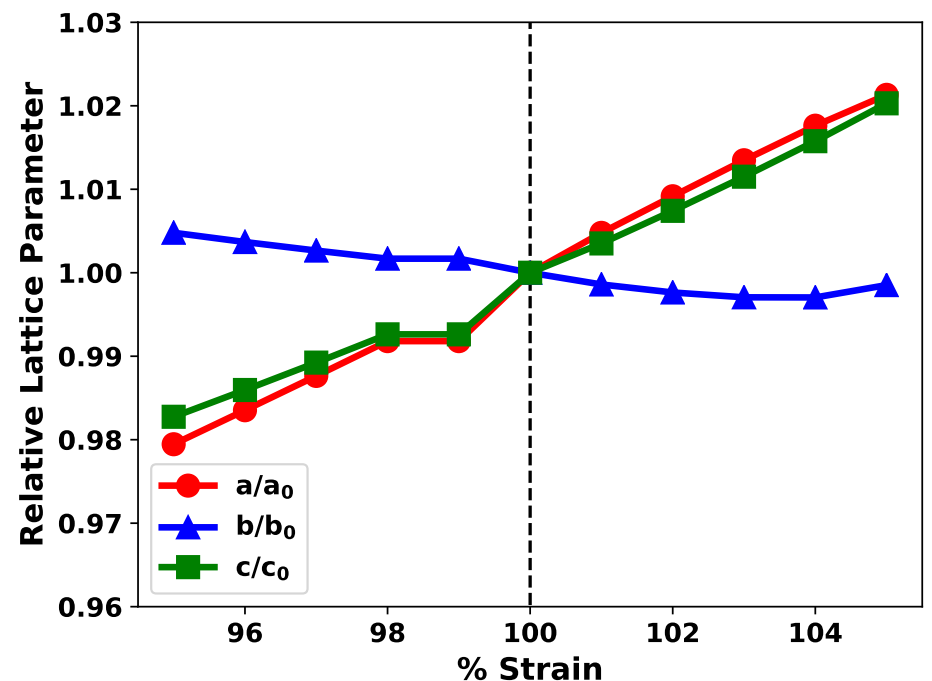

Figure 5.16: Relative change in the lattice parameters under strain applied along the $(0.75,0.45,-0.49)$ direction. Both the a-axis and c-axis have an auxetic response to the applied strain.

and above the Fermi level. At lower energies, the Ni $d$ electrons dominate. The ELF is plotted in Figures 5.20b and 5.20a The plot displays the electron density for two different orientations, the first 5.20a is in the [100] plane, and the second $5.20 \mathrm{~b}$ us in the [010] plane. These two orientations are shown to contrast the $\mathrm{Ni}$ 


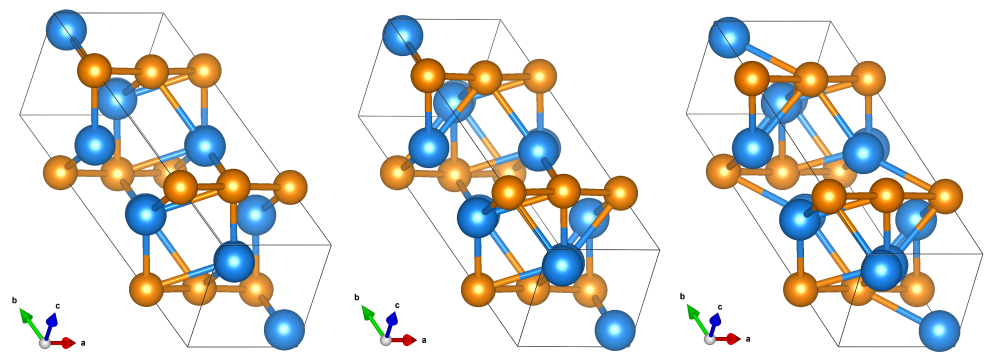

Figure 5.17: Bonding under (left) Compressive strain (middle) No strain and (right) Tensile strain.

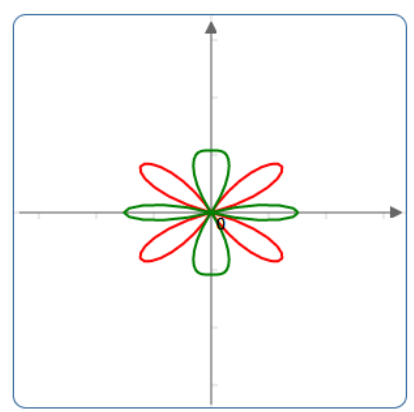

(a) xy plane

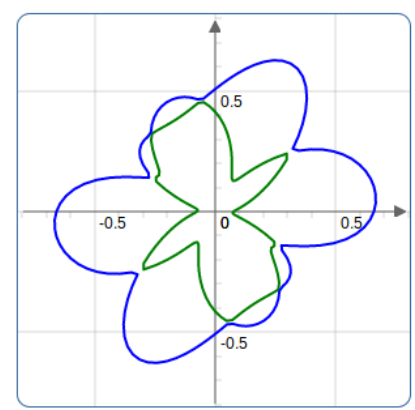

(b) xz plane

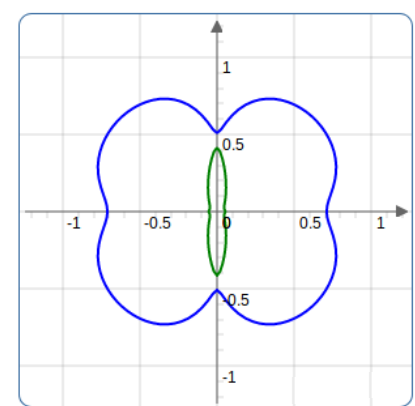

(c) yz plane

Figure 5.18: Spatial dependence of Poisson ratio for $\mathrm{NiTi}_{2}$ with space group $C 1_{2} / \mathrm{m}$. Green curves correspond to positive values and red curves to negative values. The blue curve is the contour along the maximal angle, based on a three-angle decomposition of the two vectors necessary to generate the directional depedence of the Poisson ratio.

and $\mathrm{Ti}$ atoms; there is a larger electron density between pairs of $\mathrm{Ti}$ atoms than there are between $\mathrm{Ni}$ and $\mathrm{Ti}$ atoms. This is in agreement with the calculated density of states.

\section{$5.5 \mathrm{NiTi}$}

As equiatomic NiTi is well-known for the shape-memory effect, it has likewise been very well-studied. Because of this, both the phases necessary for this effect as well as the ground-state properties have been fairly well-established. It is known that for the shape-memory effect, a low-energy martensite state with space group $P 2_{1} / m$ will transform into a higher-energy ausentite phase with space group 


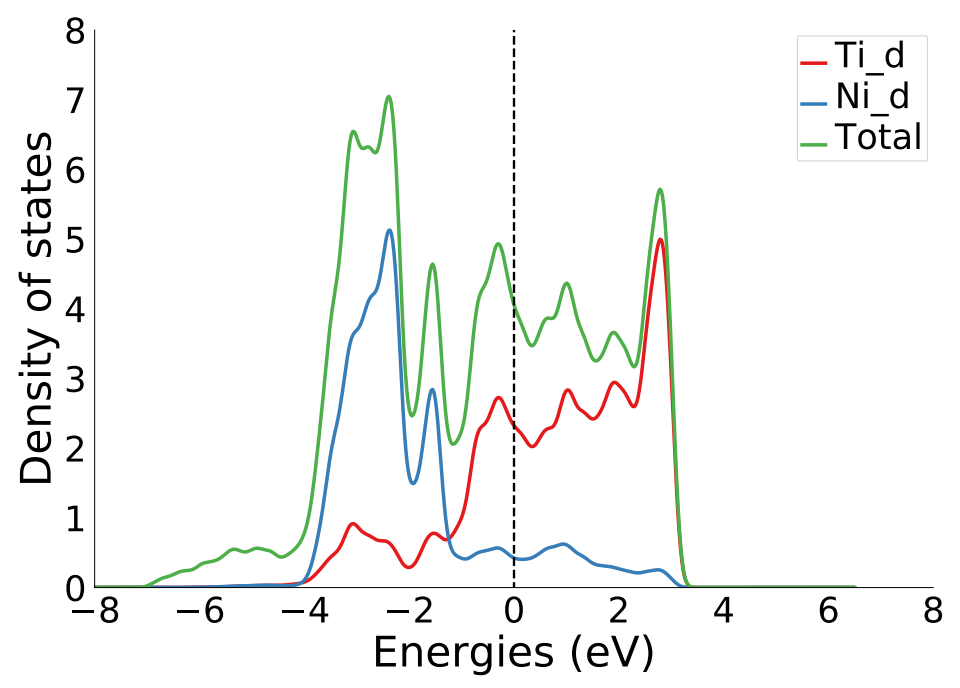

Figure 5.19: Density of states for $\mathrm{NiTi}_{2}$ with space group $C I_{2} / m$.

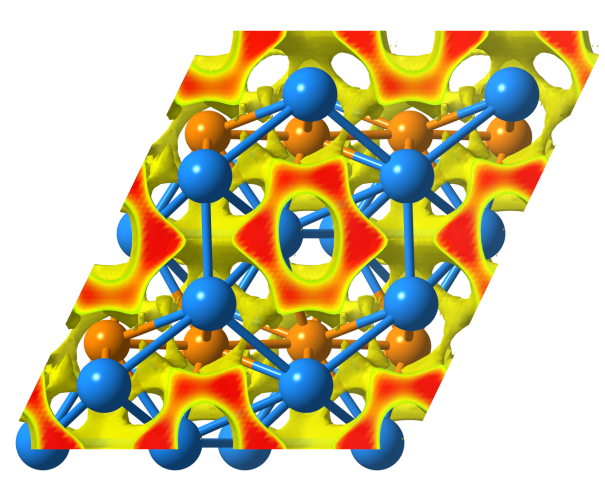

(a) Electron localization function in the $\mathrm{YZ}$ plane for $\mathrm{NiTi}_{2}$ with space group $\mathrm{Cl}_{2} / m$

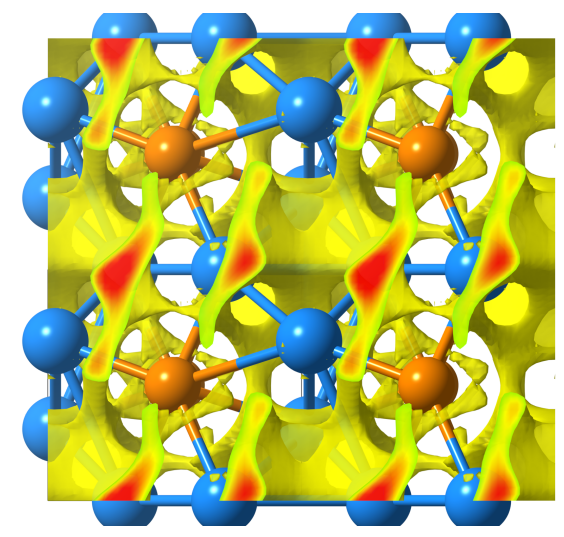

(b) Electron localization function in the $\mathrm{XZ}$ plane for $\mathrm{NiTi}_{2}$ with space group $\mathrm{Cl}_{2} / \mathrm{m}$

Figure 5.20: Electron localization function for $\mathrm{NiTi}_{2}$ with space group $\mathrm{Cl}_{2} / m$. Electrons are more localized between pairs of Ti atoms

Pm-3m. Interestingly, while the martensite phase is the experimentally found ground state of NiTi, this is not in agreement with DFT predictions. DFT predicts than orthorhombic phase with space group $\mathrm{Cncm}$ is more stable relative to the monoclinic $P 2{ }_{1} / m$ phase. In order to determine the validity of this result, 
$\mathrm{NiTi}_{2} \mathrm{Cl2} / \mathrm{m}$

\begin{tabular}{|c|c|c|}
\hline $\mathbf{a}(\AA)$ & $\mathbf{b}(\AA)$ & $\mathbf{c}(\AA)$ \\
\hline 4.61 & 4.86 & 5.11 \\
\hline
\end{tabular}

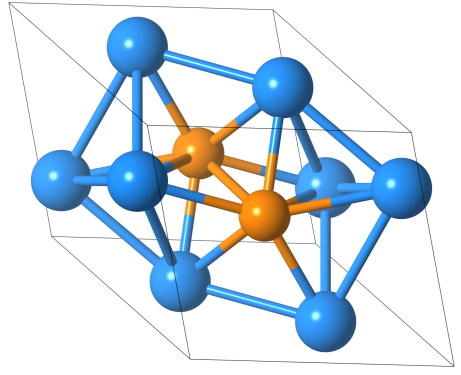

Figure 5.21: Unit cell of $\mathrm{NiTi}_{2}$ with space group $C 12 / m$.

\begin{tabular}{|c|c|c|c|c|}
\hline Atom & $\begin{array}{c}\text { Wyckoff } \\
\text { Position }\end{array}$ & $\mathrm{x}$ & $\mathrm{y}$ & $\mathrm{z}$ \\
\hline $\mathrm{Ni} 1$ & $\mathrm{a}$ & 0.50 & 0.25 & 0.50 \\
$\mathrm{Ni} 2$ & $\mathrm{a}$ & 0.50 & 0.75 & 0.49 \\
$\mathrm{Ti} 1$ & $\mathrm{a}$ & 0.31 & 0.17 & 0.03 \\
$\mathrm{Ti} 2$ & $\mathrm{a}$ & 0.69 & 0.82 & 0.03 \\
Ti3 & $\mathrm{a}$ & 0.00 & 0.34 & 0.30 \\
Ti4 & $\mathrm{a}$ & 0.00 & 0.66 & 0.70 \\
\hline
\end{tabular}

Figure 5.22: Structural information for $\mathrm{NiTi}_{2}$ with space group $\mathrm{I} / \mathrm{mmm}$.

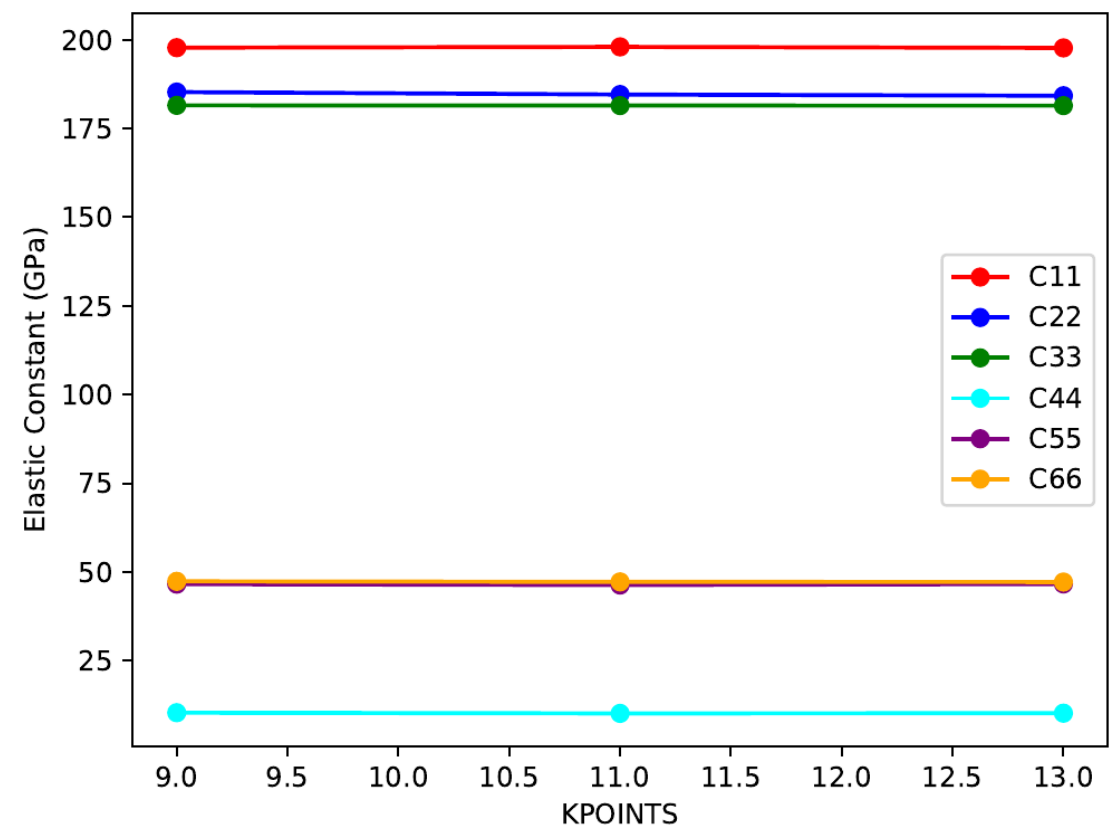

Figure 5.23: Elastic constant convergence as a function of the k-point mesh. This shows that the with a 9x9x9 k-point grid, the elastic constants are well-converged. 
Huang et. al. performed DFT calculations on these two structures in addition to the related Pnma structure, as this is the martensite structure in two other shapememory materials, PdTi and PtTi, which are driven by the martensite transformation. While the Pnma orthorhombic structure was reported by Hunag et. al, it does not appear in either OQMD nor the Materials Project. Ultimately, they found that the Pnma structure is stable relative to the monoclinic structure. This has dramatic consequences for the shape-memory effect, as it was previously assumed that there was a atom-to-atom correspondence between the monoclinic martensite and cubic austenite structures. Instead, internal stresses stabilize the monoclinic structure relative to the orthorhombic phase, meaning that the orthrhombic phase is not likely to be found experimentally. In agreement with their results, it was found to be $1.7 \mathrm{meV} /$ atom lower in energy than the monoclinic structure. It must be emphasized that this energetic difference is rather small. Overall, results are reported which are in agreement with previous experimental and theoretical results. The structural information for each of the four structures described above is listed in Tables 5.31, 5.27, 5.29, and 5.33. To assess dynamical stability, phonon calculations were performed using the Phonopy software package. Stable phonons were found for both orthrhombic phases as well as the monoclinc phase, which demonstrates dynamic stability. For the cubic phase, there is a large negative phonon mode around the M symmetry point, which demonstrates that the cubic phase is not thermally stable at 0 temperature. This is not surprising, as the cubic phase is only stabilized at higher temperatures. In addition, this phonon spectrum is in agreement with previously reported theoretical calculations. The phonon spectra for each structures are displayed in Figures 5.34c, 5.34a, 5.34b, and 5.34d.

The band structures of each orthrhombic phase are show in Figures 5.24 and 5.25. In each case, the d-orbital contributions are projected for both $\mathrm{Ni}$ and $\mathrm{Ti}$ atoms. In the case of the Pnma phase, the d-orbitals of both atoms are below the Fermi level. In the $\mathrm{Cncm}$ phase, The d-orbital contirbutions from the Ni atoms are below the Fermi level, but the d-orbital contributions from Ti span a range of -1 $\mathrm{eV}$ to $4 \mathrm{eV}$.

In addition a metastable phase for NiTi with space group $P 2 / m$ was located with NLC. With the use of the ELATE software package [33], the directional dependence of the elastic properties was determined. While the average values of each are positive, they are quite anisotropic. From this analysis, NLC attains a maximal negative value in the a-c plane. The maximal value of NLC is -4.1732 $\mathrm{TPa}^{-1}$, and is directed along the unit vector $(-0.89,0.00,-0.46)$. In the rutile structure class, $\mathrm{TeO}_{2}$ displays the maxmimal $\mathrm{NLC}$ value of $-5.1 \mathrm{TPa}^{-1} \cdot \mathrm{BiB}_{3} \mathrm{O}_{6}$, which displays NLC due to a tilted network mechanism, has been shown experimentally 


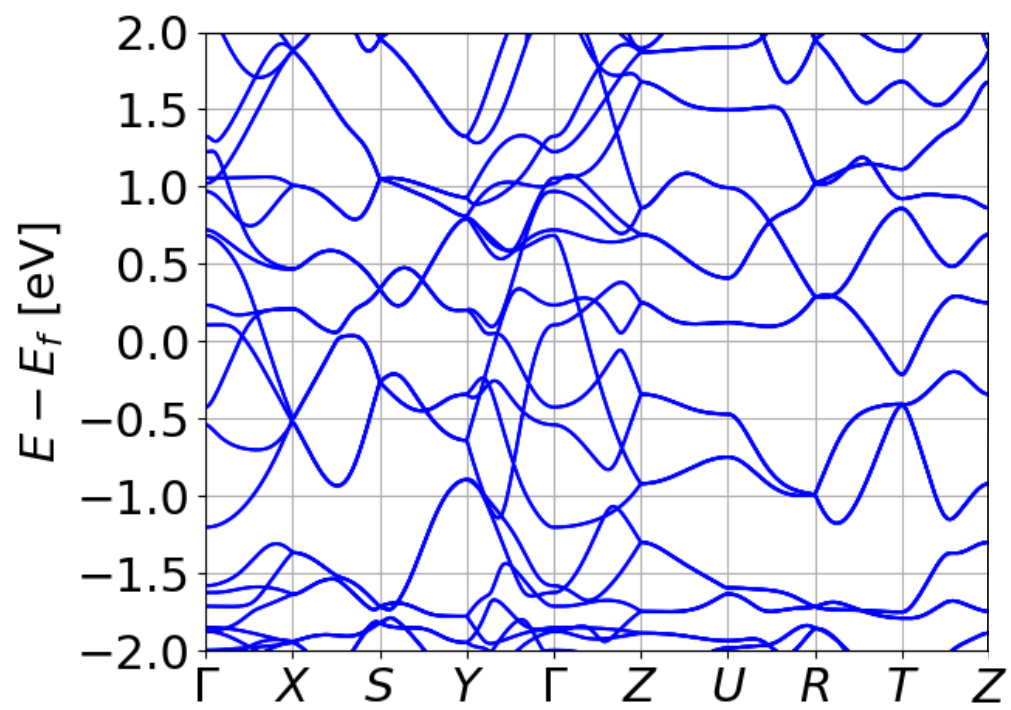

Figure 5.24: Band structure for NiTi with space group $\mathrm{Cncm}$. Since the conduction bands cross at the Fermi level, this structure is a conductor.

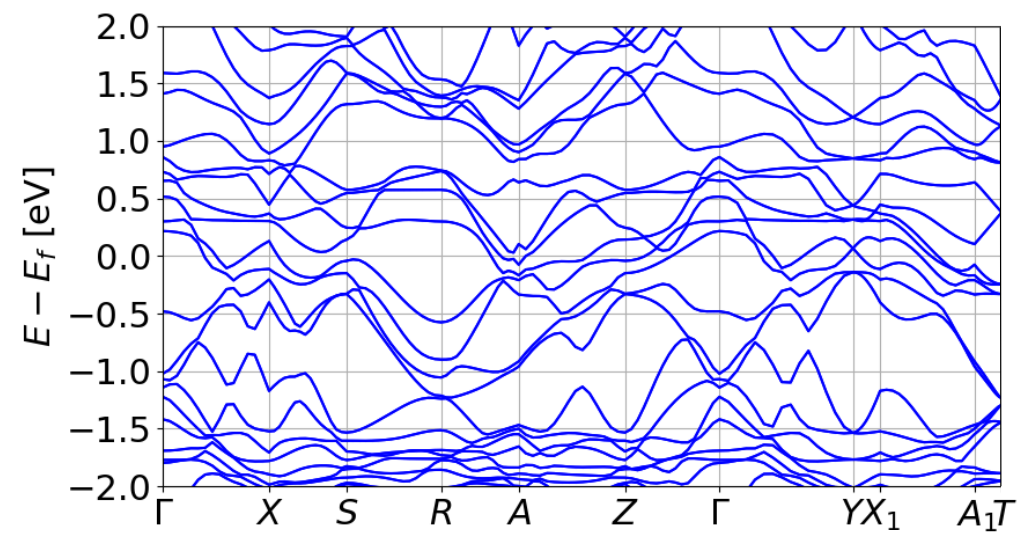

Figure 5.25: Band structure for NiTi with space group Pnma. Since the conduction bands cross at the Fermi level, this structure is a conductor.

to have NLC equal to $-12.5 \mathrm{TPa}^{-1}$. However, a much larger value of $42 \mathrm{TPa}^{-1}$ was found in zinc dicyanoaurate $\mathrm{Zn}\left[\mathrm{Au}(\mathrm{CN})_{2}\right]_{2}$ [17]. Thus, this structure has a robust NLC that is comparable in value to previously discovered crystals. To show the mechanism is not unique to the particular choice of $\mathrm{Ni}$ and $\mathrm{Ti}$, additional calculations were performed with $\mathrm{Ni}$ and $\mathrm{Ti}$ replaced with other elements common to 


\begin{tabular}{|c|c|c|}
\hline \multicolumn{3}{|c|}{ NiTi Pnma } \\
\hline $\mathbf{a}(\stackrel{\circ}{A})$ & $\mathbf{b}(\stackrel{\circ}{A})$ & c $(\stackrel{\circ}{A})$ \\
\hline 5.20 & 4.10 & 5.12 \\
\hline
\end{tabular}

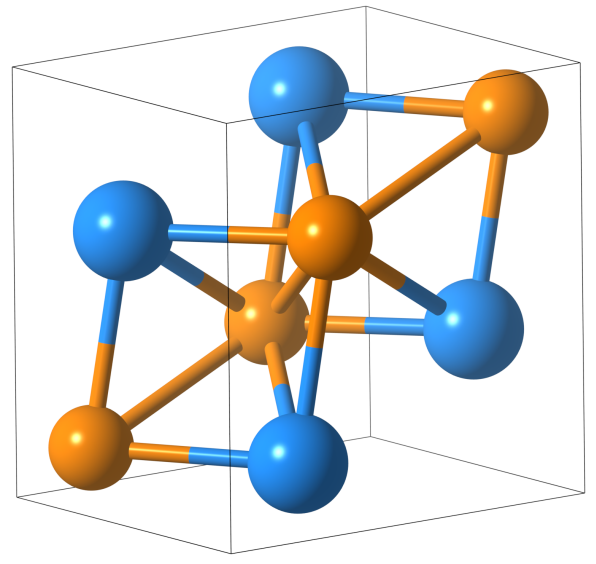

Figure 5.26: Unit cell of NiTi with space group Pnma

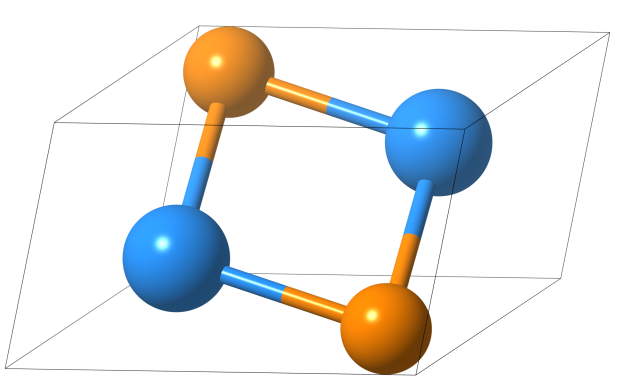

Figure 5.28: Unit cell of NiTi with space group $\mathrm{Cmcm}$

\begin{tabular}{|c|c|c|c|c|}
\hline Atom & $\begin{array}{c}\text { Wyckoff } \\
\text { Position }\end{array}$ & $\mathrm{x}$ & $\mathrm{y}$ & $\mathrm{z}$ \\
\hline $\mathrm{Ni} 1$ & $\mathrm{c}$ & 0.44 & 0.35 & 0.36 \\
$\mathrm{Ni} 2$ & $\mathrm{c}$ & 0.06 & 0.75 & 0.86 \\
$\mathrm{Ni} 3$ & $\mathrm{c}$ & 0.56 & 0.75 & 0.64 \\
$\mathrm{Ni} 4$ & $\mathrm{c}$ & 0.94 & 0.25 & 0.14 \\
$\mathrm{Ti} 1$ & $\mathrm{c}$ & 0.84 & 0.25 & 0.63 \\
$\mathrm{Ti} 2$ & $\mathrm{c}$ & 0.66 & 0.75 & 0.13 \\
$\mathrm{Ti} 3$ & $\mathrm{c}$ & 0.16 & 0.75 & 0.37 \\
$\mathrm{Ti} 4$ & $\mathrm{c}$ & 0.34 & 0.25 & 0.87 \\
\hline
\end{tabular}

Figure 5.27: Structural information for NiTi with space group Pnma

\begin{tabular}{|c|c|c|}
\multicolumn{3}{|c}{ NiTi Cmcm } \\
\hline $\mathbf{a}(\stackrel{\circ}{A})$ & b $(\stackrel{\circ}{A})$ & $\mathbf{c}(\stackrel{\circ}{A})$ \\
\hline 2.93 & 4.00 & 4.90 \\
\hline
\end{tabular}

\begin{tabular}{|c|c|c|c|c|}
\hline Atom & $\begin{array}{c}\text { Wyckoff } \\
\text { Position }\end{array}$ & $\mathrm{x}$ & $\mathrm{y}$ & $\mathrm{z}$ \\
\hline Ni1 & $\mathrm{c}$ & 0.08 & 0.75 & 0.83 \\
$\mathrm{Ni} 2$ & $\mathrm{c}$ & 0.91 & 0.25 & 0.17 \\
Ti1 & $\mathrm{c}$ & 0.36 & 0.75 & 0.29 \\
Ti2 & $\mathrm{c}$ & 0.64 & 0.25 & 0.72 \\
\hline
\end{tabular}

Figure 5.29: Structural information for NiTi with space group $\mathrm{Cmcm}$

intermetallics. The elements chosen where $\mathrm{Ag}, \mathrm{Cd}, \mathrm{Cu}, \mathrm{Zn}, \mathrm{Mn}$, and $\mathrm{Nb}$. Out of all substitutions performed, only NiMn yielded a stable structure with the same crystalline lattice. The directional dependence of the linear compressibility are displayed in Figure 5.36. The green curves show where the linear compressbility is positive, and the red curves where it is negative. NiMn also displays NLC, which is similar in character to NiTi. It appears that Ni is critical to this effect, as 


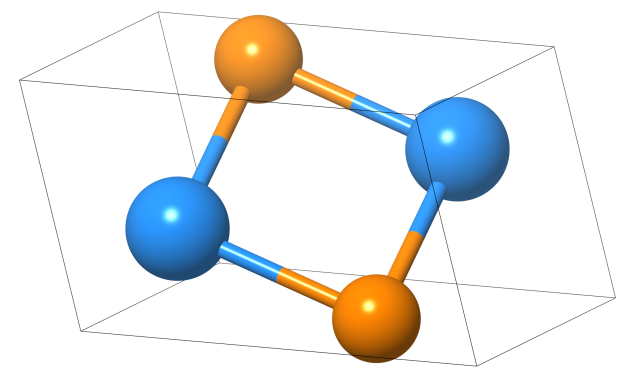

Figure 5.30: Unit cell of NiTi with space group $P 2{ }_{1} / m$

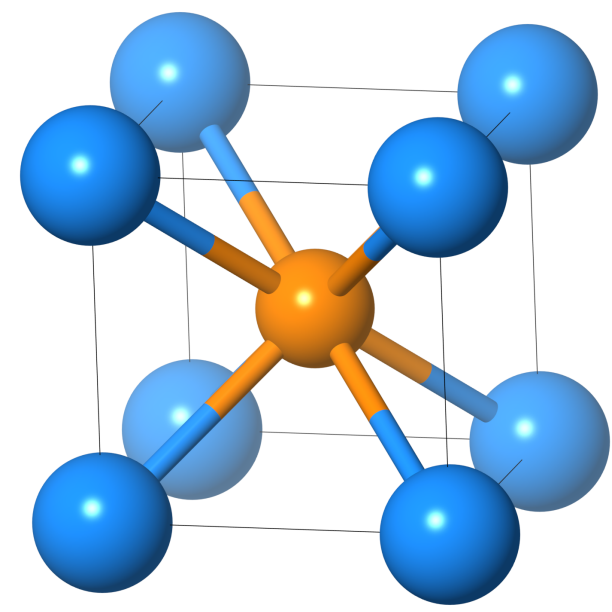

Figure 5.32: Unit cell of NiTi with space group $\mathrm{Pm}-3 \mathrm{~m}$

\begin{tabular}{|c|c|c|}
\multicolumn{3}{|c}{ NiTi $\boldsymbol{P} 2_{1} / \boldsymbol{m}$} \\
\hline $\mathbf{a}(\AA)$ & b $(\stackrel{\circ}{A})$ & $\mathbf{c}(\stackrel{\circ}{ })$ \\
\hline 4.93 & 4.01 & 2.91 \\
\hline
\end{tabular}

\begin{tabular}{|c|c|c|c|c|}
\hline Atom & $\begin{array}{c}\text { Wyckoff } \\
\text { Position }\end{array}$ & $\mathrm{x}$ & $\mathrm{y}$ & $\mathrm{z}$ \\
\hline Ni1 & $\mathrm{e}$ & 0.67 & 0.25 & 0.09 \\
Ni2 & $\mathrm{e}$ & 0.33 & 0.75 & 0.91 \\
Ti1 & $\mathrm{e}$ & 0.21 & 0.25 & 0.35 \\
Ti2 & $\mathrm{e}$ & 0.78 & 0.75 & 0.65 \\
\hline
\end{tabular}

Figure 5.31: Structural information for NiTi with space group $P 21 / m$

\begin{tabular}{|c|c|c|}
\hline \multicolumn{3}{|c}{ NiTi Pm-3m } \\
\hline a $(\stackrel{\circ}{A})$ & b $(\stackrel{\circ}{A})$ & $\mathbf{c}(\stackrel{\circ}{A})$ \\
\hline 4.93 & 4.01 & 2.91 \\
\hline
\end{tabular}

\begin{tabular}{|c|c|c|c|c|}
\hline Atom & $\begin{array}{c}\text { Wyckoff } \\
\text { Position }\end{array}$ & $\mathrm{x}$ & $\mathrm{y}$ & $\mathrm{z}$ \\
\hline Ni1 & $\mathrm{e}$ & 0.67 & 0.25 & 0.09 \\
Ni2 & $\mathrm{e}$ & 0.33 & 0.75 & 0.91 \\
Ti1 & $\mathrm{e}$ & 0.21 & 0.25 & 0.35 \\
Ti2 & $\mathrm{e}$ & 0.78 & 0.75 & 0.65 \\
\hline
\end{tabular}

Figure 5.33: Structural information for NiTi with space group $\mathrm{Pm}-3 \mathrm{~m}$

it is common to both phases which display NLC.

Constrained relaxation of the crystal structures within DFT allows us to determine the changes in inter-atomic distances and cell-parameters under applied hydrostatic pressure. Pressure in the range of 0-100 GPa was applied in increments of $20 \mathrm{GPa}$. Under positive pressure, the unit cell increases in length in the a and c-directions, while it decreases in the b direction. An increase in a and c cell parameters would indicate an increase in area, but, as stress is applied, the angle between a and c decreases sufficiently to yield an overall area in the a-c 

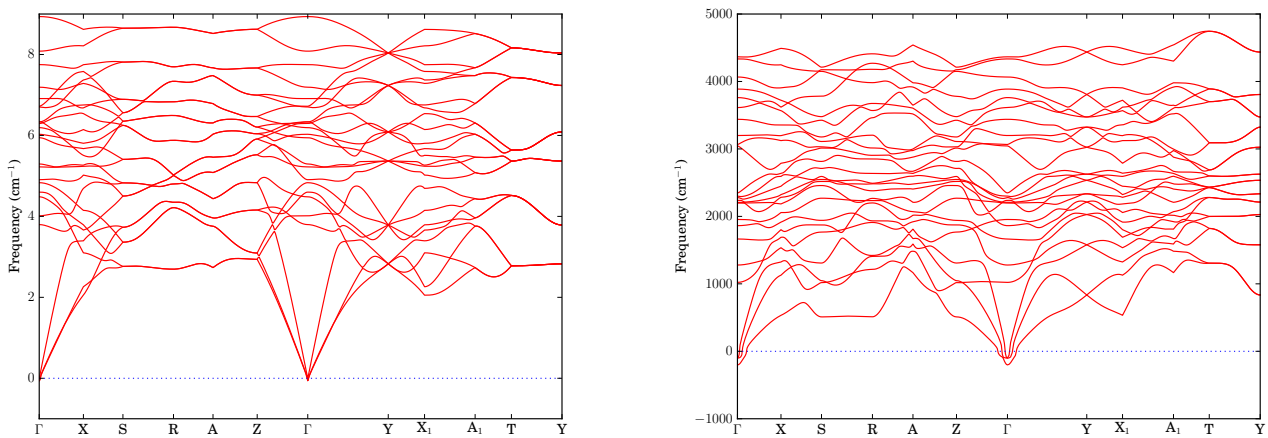

(a) Phonons for NiTi with space group Pnma

(b) Phonons for NiTi with space group $\mathrm{Cncm}$

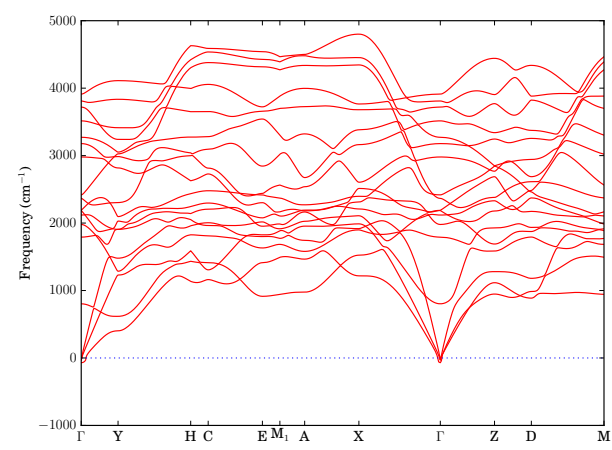

(c) Phonons for NiTi with space group $P 2{ }_{1} / m$

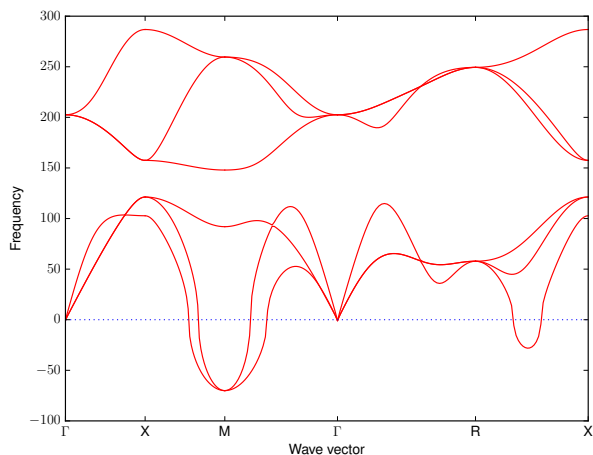

(d) Phonon spectrum for equiatomic NiTi with space group $P m-3 m$

Figure 5.34: Phonon modes for structures found using MHM for NiTi. All calculations were performed at $0 \mathrm{~K}$. The $P m-3 m$ has a large negative mode at the $\mathrm{M}$ point, but this is to be expected as it is the high-energy austenite phase; this structure is stabilized at higher temperatures.

plane which shrinks. This implies that the NLC is not along the crystalline axes (in agreement with the direction of maximal NLC found with ELATE), which is unsurprising, as the structure has monoclinic symmetry [16]. The mechanism for NLC is displayed in Figure 5.37. Under hydrostatic strain, Ni atoms in the [101 plane are forced closer together. This forces the Ti atoms in this plane further apart; this is precisely the same as the well-known wine-rack model. The ELFCAR plot in Figure 5.38 shows why this occurs. There is strong electron localization between pairs of $\mathrm{Ti}$ atoms in the [101] direction, which is lacking between 


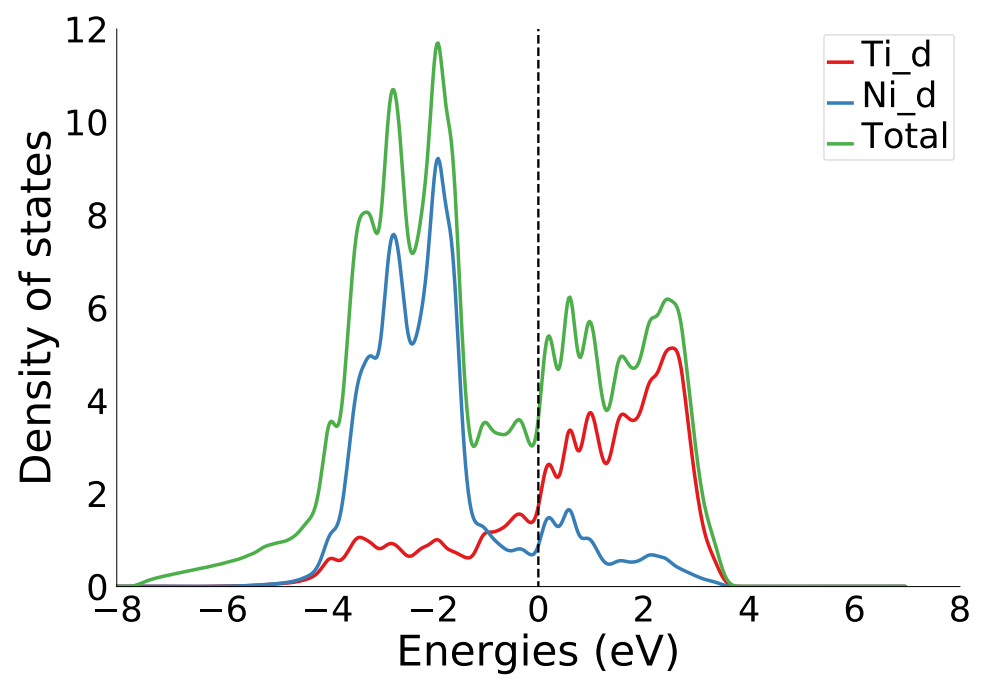

Figure 5.35: Density of states for NiTi with space group Pnma.

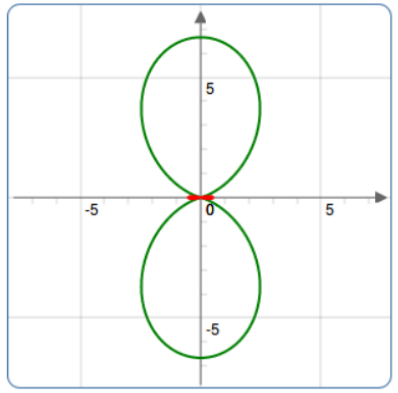

(a) xy plane

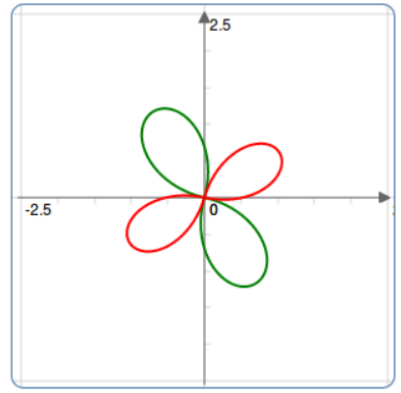

(b) xz plane

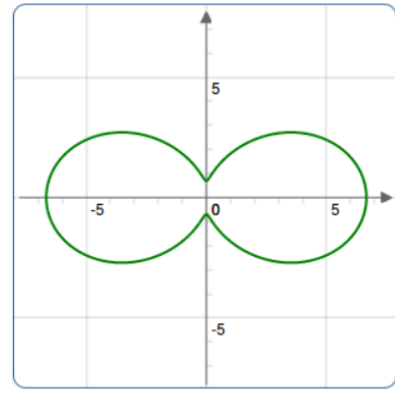

(c) yz plane

Figure 5.36: Directional dependence of the linear compressibility for NiTi with space group $P 2 / m$. Green curves display positive linear compressibility, and red curves display negative linear compressibility. The maximal negative value occurs in the $\mathrm{XZ}$ plane.

pairs of $\mathrm{Ni}$ atoms. Under hydrostatic strain. the $\mathrm{Ni}$ atoms are forced closer together. Due to the strong bonding between Ti atoms along the [101], the distance between them remains fixed. This contraction between $\mathrm{Ni}$ atoms in turn forces $\mathrm{Ti}$ atoms separated along the [-102] direction further apart. As further evidence for the effect, the Ti d-states are more highly occupied at the Fermi level, displayed in Figure 5.40 . 

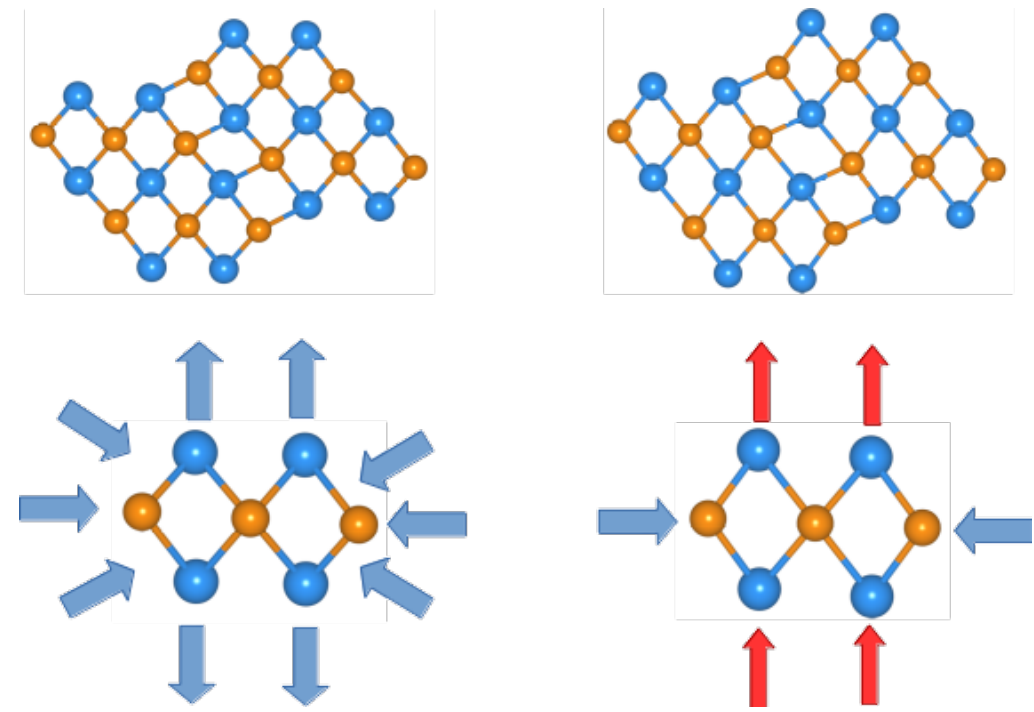

Figure 5.37: Mechanism for NLC for NiTi with space group $P 2 / m$. The mechanism is equivalent to the wine-rack model.

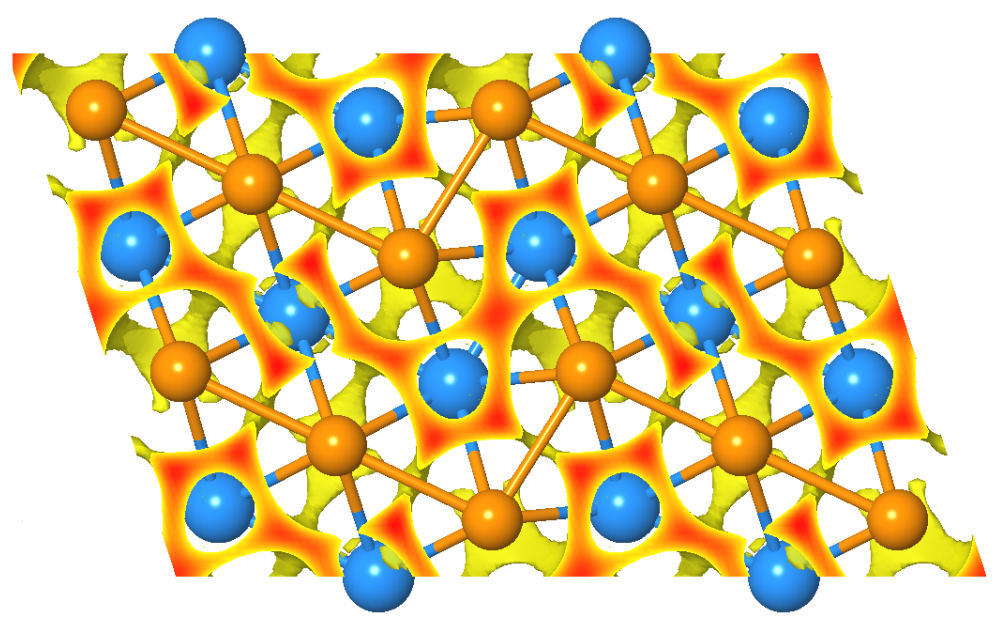

Figure 5.38: ELF for NiTi with space group $P 2_{1} / m$. Along the diagonally arranged Ni atoms, there is strong electron localization indicating that these bonds are strong.

\section{$5.6 \quad \mathrm{Ni}_{3} \mathrm{Ti}$}

For $\mathrm{Ni}_{3} \mathrm{Ti}$, two structures were found which are nearly energy degenerate, a hexagonal structure with space group $\mathrm{P}_{3} / \mathrm{mmc}$ and a cubic structure with space group 


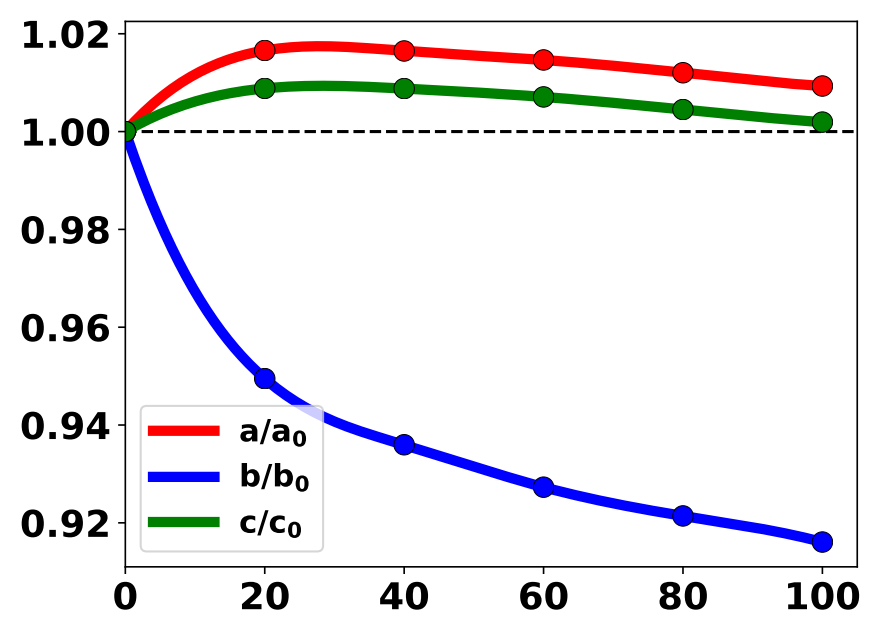

Figure 5.39: Relative change in the lattice parameters under hydrostatic pressures of $2,4,6,8$, and $10 \mathrm{GPa}$ for $\mathrm{Ni}_{1} \mathrm{Ti}_{1}$. The increase in a and $\mathrm{b}$ lattice parameters indicates that this structure has NLC.

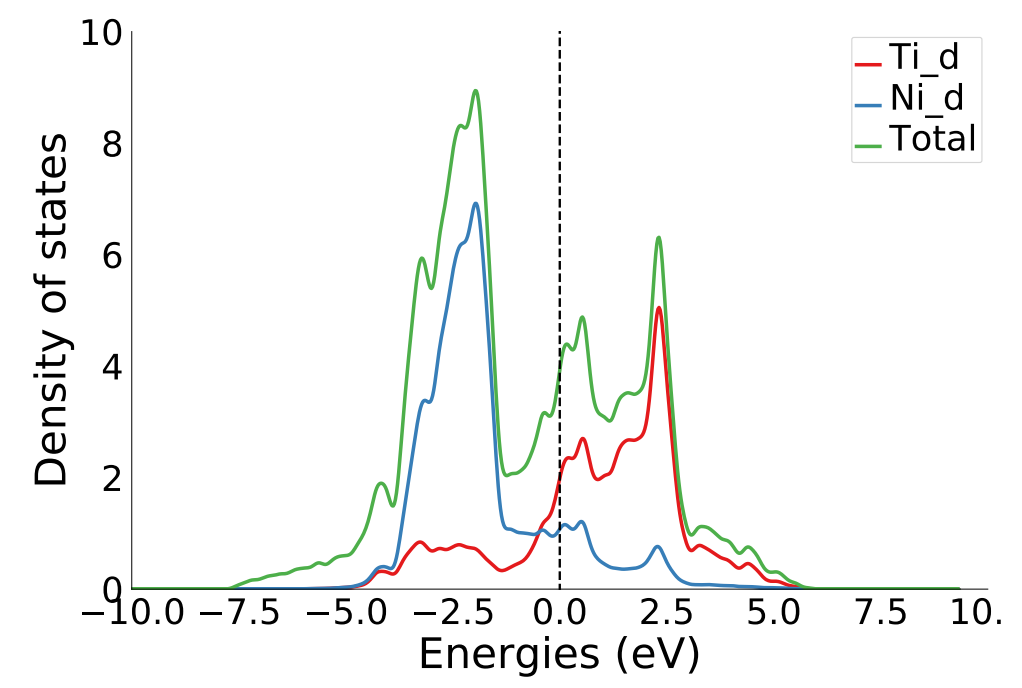

Figure 5.40: Density of states of NiTi with space group $P 2 / m$. Hybridization of Ti and Ni states occurs at the Fermi level.

Pm-3m. Both of these structures were previously reported in both the Materials Project and OQMD. The structural information for both are listed in Tables 5.42 
and 5.44. The phonon spectra for each structure is displayed in Figures $5.45 \mathrm{a}$ and $5.45 \mathrm{~b}$. Since the phonon modes are positive for both phases, both are dynamically stable.

The Born conditions are satisfied for both structures, indicating mechanical stability. The elastic constants are displayed in Table 5.2.

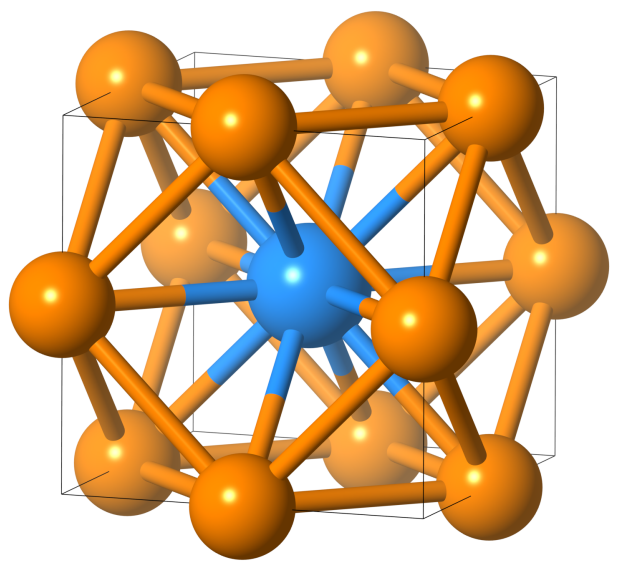

Figure 5.41: Unit cell of $\mathrm{Ni}_{3} \mathrm{Ti}$ with space group $\mathrm{P}_{3} / \mathrm{mmc}$

\begin{tabular}{|c|c|c|}
\multicolumn{3}{|c}{$\mathbf{N i}_{3} \mathbf{T i}$ P6 $_{3} / \mathbf{m m c}$} \\
\hline $\mathbf{a}(\stackrel{\circ}{A})$ & $\mathbf{b}(\stackrel{\circ}{A})$ & $\mathbf{c}(\stackrel{\circ}{A})$ \\
\hline 5.10 & 4.17 & 5.10 \\
\hline
\end{tabular}

\begin{tabular}{|c|c|c|c|c|}
\hline Atom & $\begin{array}{c}\text { Wyckoff } \\
\text { Position }\end{array}$ & $\mathrm{x}$ & $\mathrm{y}$ & $\mathrm{z}$ \\
\hline $\mathrm{Ni} 1$ & $\mathrm{~b}$ & 0.67 & 0.50 & 0.84 \\
$\mathrm{Ni} 2$ & $\mathrm{~b}$ & 0.16 & 0.50 & 0.32 \\
$\mathrm{Ni3}$ & $\mathrm{a}$ & 0.84 & 0.00 & 0.68 \\
Ni4 & $\mathrm{a}$ & 0.84 & 0.00 & 0.16 \\
Ni5 & $\mathrm{a}$ & 0.32 & 0.00 & 0.16 \\
Ni6 & $\mathrm{b}$ & 0.16 & 0.50 & 0.84 \\
Ti1 & $\mathrm{b}$ & 0.66 & 0.50 & 0.33 \\
Ti2 & $\mathrm{a}$ & 0.33 & 0.00 & 0.66 \\
\hline
\end{tabular}

Figure 5.42: Structural information for $\mathrm{Ni}_{3} \mathrm{Ti}$ with space group $\mathrm{P}_{3} / \mathrm{mmc}$

\section{$5.7 \quad \mathbf{N i}_{5} \mathbf{T i}$}

A previously unreported structure was found on the convex hull with space group Cmmm. This structure is just barely on the convex hull. This would entail that any synthesis of this structure would likely result in an arbitrary mixture of $\mathrm{Ni}, \mathrm{Ni}_{3} \mathrm{Ti}$ and $\mathrm{Ni}_{5} \mathrm{Ti}$. Nonetheless, it is a new structure which is on the convex hull. The structural information is listed in Table 5.47 From the phonon band structure in Figure 5.48, the structure is dynamically stable. The slight negativity at $\Gamma$ is for the same reasons as the hexagonal $\mathrm{Ni}_{3}$ Ti structure. The bands stucture is displayed in Figure 5.51. Additionally, the elastic constants satisfy the Born criteria, indicating that the structure is also mechanically stable. Of interest are the structure's elastic properties, which are listed in Table 5.2. The band The elastic properties of this phase are enhanced in comparison to pristine $\mathrm{Ni}$ and pristine $\mathrm{Ti}$. 


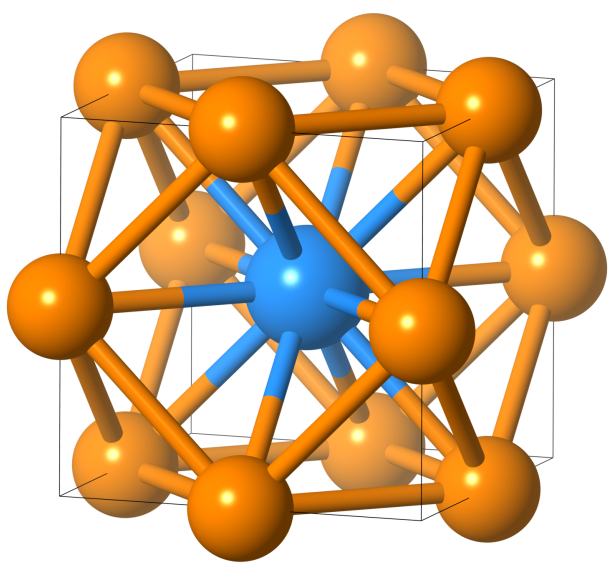

Figure 5.43: Unit cell of $\mathrm{Ni}_{3} \mathrm{Ti}$ with space group $P m-3 m$

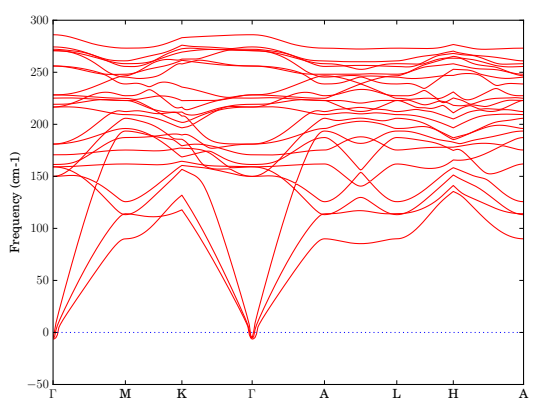

(a) Phonon modes for $\mathrm{Ni}_{3} \mathrm{Ti}$ with space group $\mathrm{P}_{3} / m m c$. Since this structure satisfies the Born criteria for mechanical stability, the slight negativity at the $\Gamma$ point is a convergence issue; all modes are positive so this structure is dynami-

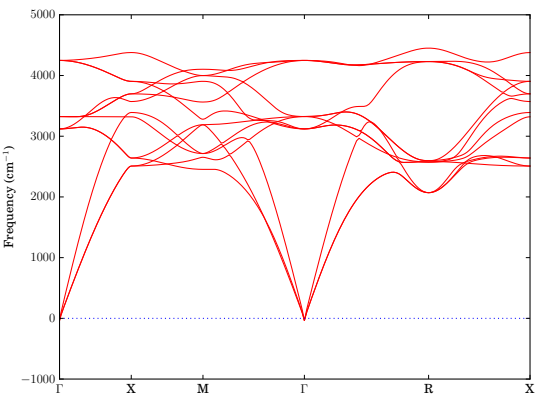

(b) Phonon modes for $\mathrm{Ni}_{3} \mathrm{Ti}$ with space group Pm-3m. Since all phonon modes are positive, this structure is dynamically stable cally stable.

This enhancement primarily stems Ni-Ni bonds in the [001] plane. This can be seen from the projected Crystal Orbital Hamiltonian Population plot (pCOHP) displayed in Figure 5.50, pCOHP is a method which is used to partition the bandstructure energy as a sum of orbital pair contributions. This allows the generation 


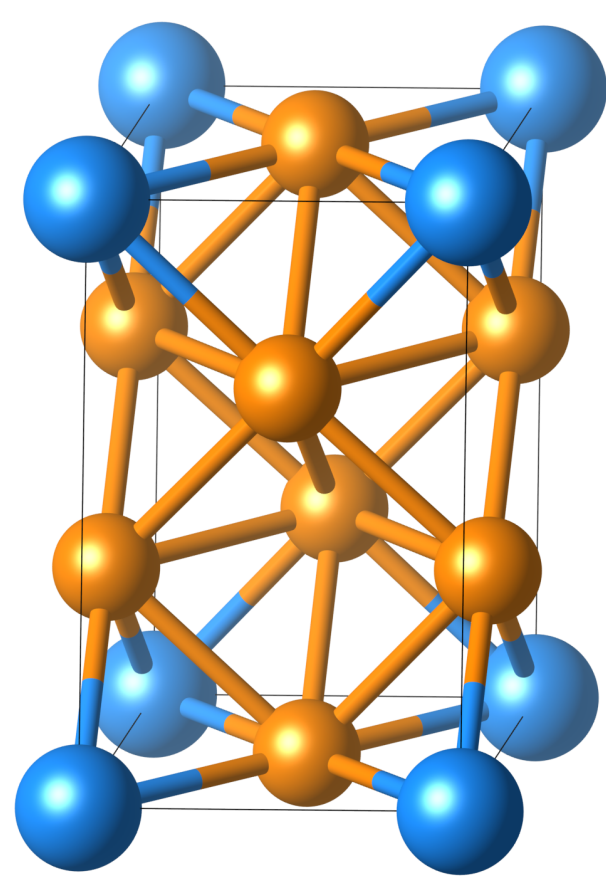

Figure 5.46: Unit cell of $\mathrm{Ni}_{5} \mathrm{Ti}$ with space group $\mathrm{Cmmm}$

\begin{tabular}{|c|c|c|c|c|}
\hline \multicolumn{5}{|c|}{$\mathrm{NiTi}_{5} \mathrm{Cmmm}$} \\
\hline $\mathbf{a}(\stackrel{\circ}{A})$ & \multicolumn{2}{|c|}{$\mathbf{b}(\stackrel{\circ}{A})$} & \multicolumn{2}{|c|}{$\mathbf{c}(\stackrel{\circ}{A})$} \\
\hline 3.57 & \multicolumn{2}{|c|}{3.61} & \multicolumn{2}{|c|}{5.63} \\
\hline Atom & $\begin{array}{l}\text { Wyckoff } \\
\text { Position }\end{array}$ & $\mathrm{X}$ & $\mathrm{y}$ & $\mathrm{Z}$ \\
\hline Ni1 & J & 0.17 & 0.50 & 0.33 \\
\hline $\mathrm{Ni} 2$ & $\mathrm{j}$ & 0.83 & 0.50 & 0.66 \\
\hline $\mathrm{Ni3}$ & $\mathrm{c}$ & 0.50 & 0.50 & 0.00 \\
\hline $\mathrm{Ni} 4$ & $\mathrm{i}$ & 0.66 & 0.00 & 0.33 \\
\hline $\mathrm{Ni} 5$ & $\mathrm{i}$ & 0.33 & 0.00 & 0.66 \\
\hline Ti1 & a & 0.00 & 0.00 & 0.00 \\
\hline
\end{tabular}

Figure 5.47: Structural information for $\mathrm{Ni}_{5} \mathrm{Ti}$ with space group $\mathrm{Cmmm}$

of a -pCOHP diagram which indicated bonding (positive), anti-bonding (negative), and non-bonding (zero) energy regions. In this plot, the contributions between pairs of $\mathrm{Ni}$ and $\mathrm{Ti}$ atoms is displayed. At the Fermi energy, contributions from pairs of $\mathrm{Ni}$ atoms in the [001] plane dominate, which can be more easily seen in the inset plot. This can be further seen from the Electron Localization Function (ELF) plot in Figure 5.49, as there is strong electron localization in between pairs of Ni atoms. The density of states plot in Figure 5.52 provides further evidence for these bonds. The calculated electron densities are the same in both spin channels, so the phase is non-magnetic. In addition, the total DOS in this figure is the sum of both spin-channels. At the Fermi level, occupied Ni d-states are the dominant contribution.

In addition to this new phase on the convex hull, a cubic phase with space group $F-43 m$ and a monoclinc phase with space group $C 2 / m$ were found for $\mathrm{Ni}_{5} \mathrm{Ti}$. These phases are of interest due to the nature of the martenistic transformation in near equiatomic NiTi, as it entails a low-energy monoclinc phase being transformed into a higher-energy cubic phase. Similarly, the low-energy phase for NiTi 


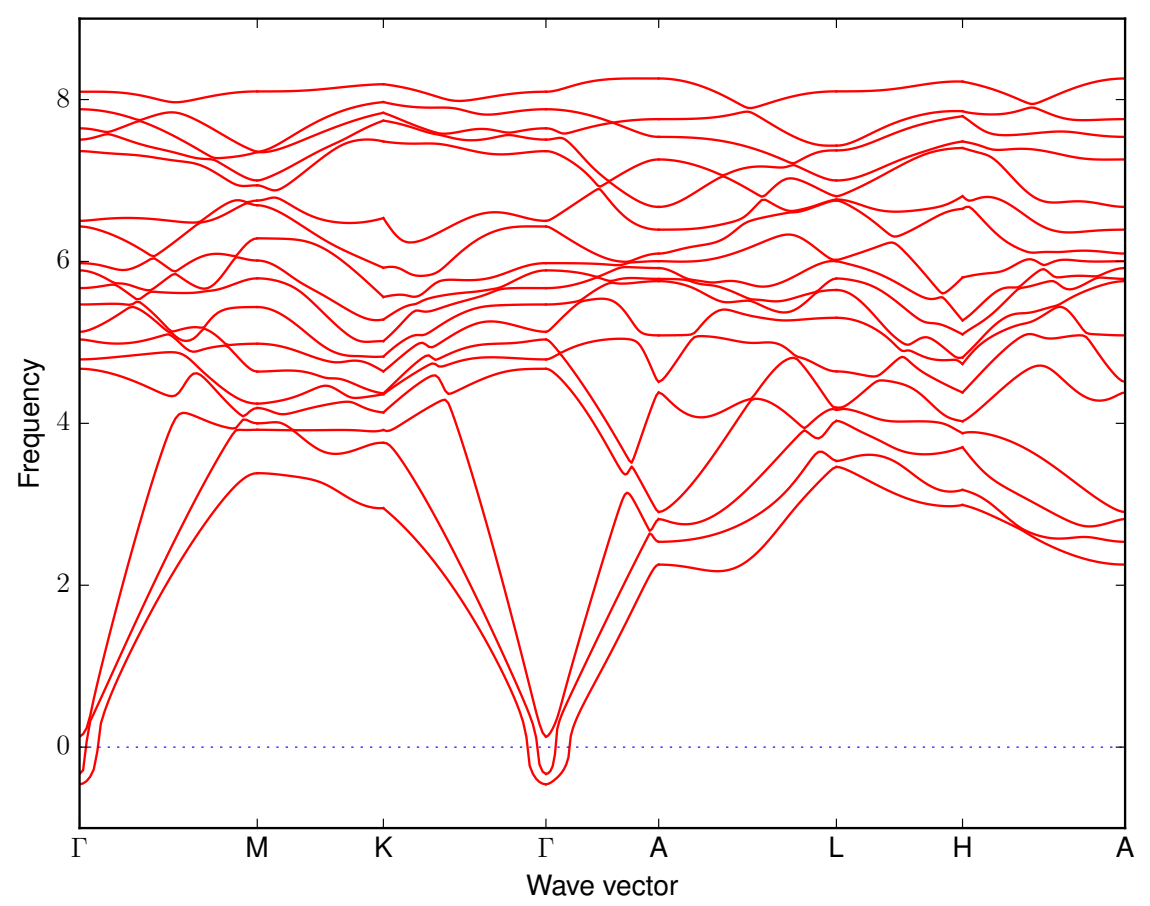

Figure 5.48: Phonons for $\mathrm{Ni}_{5} \mathrm{Ti}$ with space group $\mathrm{Cmmm}$. Since this structure satisfies the Born criteria for mechanical stability, the slight negativity at the $\Gamma$-point is a convergence issue; the structure is dynamically stable.

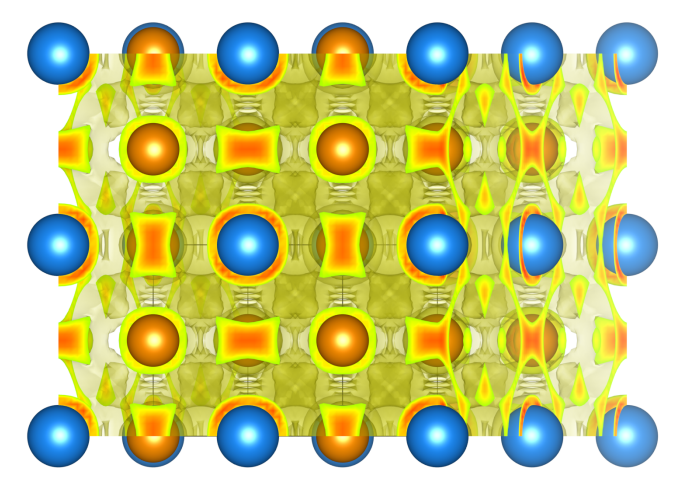

Figure 5.49: ELF for $\mathrm{Ni}_{5} \mathrm{Ti}$ with space group Cmmm. Dense electron localization between the $\mathrm{Ni}$ atoms indicates that this structure has strong Ni-Ni bonds. 


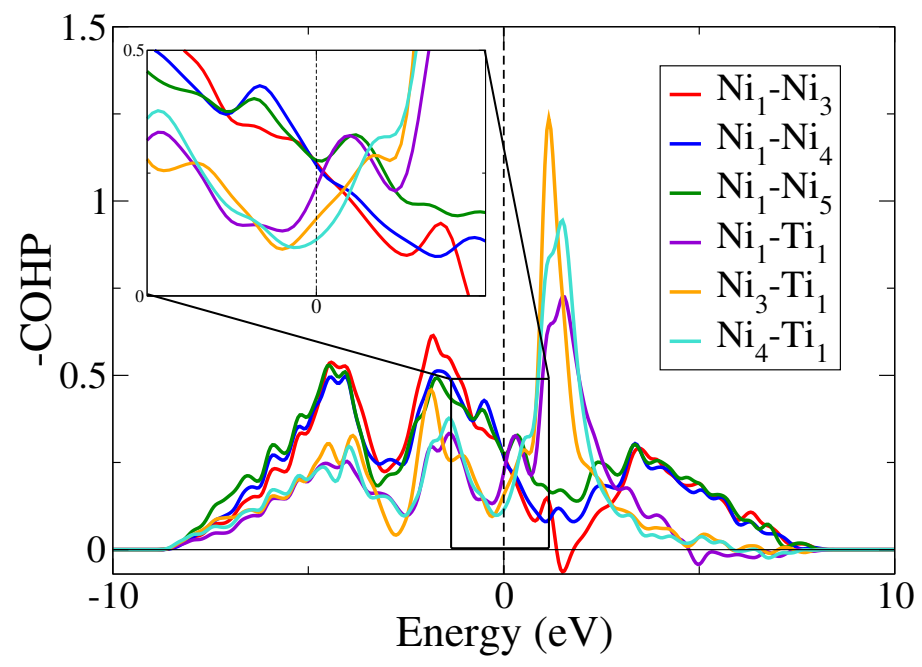

Figure 5.50: pCOHP plot for $\mathrm{Ni}_{5} \mathrm{Ti}$ with space group Cmmm. Ni-Ni contributions are dominant at the Fermi level.

is an orthorhombic phase. However, since $\mathrm{Ni}_{5} \mathrm{Ti}$ is just barely on the convex hull, it is unlikely that such a phase could be realized in isolation, as a material made with the composition will likely be a combination of $\mathrm{Ni}_{5} \mathrm{Ti}, \mathrm{Ni}_{3} \mathrm{Ti}$, and pristine $\mathrm{Ni}$.

\subsection{Chapter Summary}

Structural search by the means of minima hopping was performed for forty compositions of the binary system NiTi. These forty compositions were determined through the constraint that at most ten atoms per unit cell were considered. From this search, the resulting convex hull was mostly in agreement with previous results. The only differences were a new phase was found on the convex for $\mathrm{NiTi}_{2}$ was located with space group $14 / \mathrm{mmm}$. This differs from the previously reported cubic structure with space group $F m-3 m$. This phase is of interest not only because it is on the convex hull. but also because it has flat bands at the $\Gamma$-point. Due to this, the Fermi surface of this phase is two nested cylinders. Additionally, a new composition appears on the convex hull, $\mathrm{Ni}_{5} \mathrm{Ti}$, with space group Cmmm. However, this structure is only barely on the convex hull, so any attempts to create 


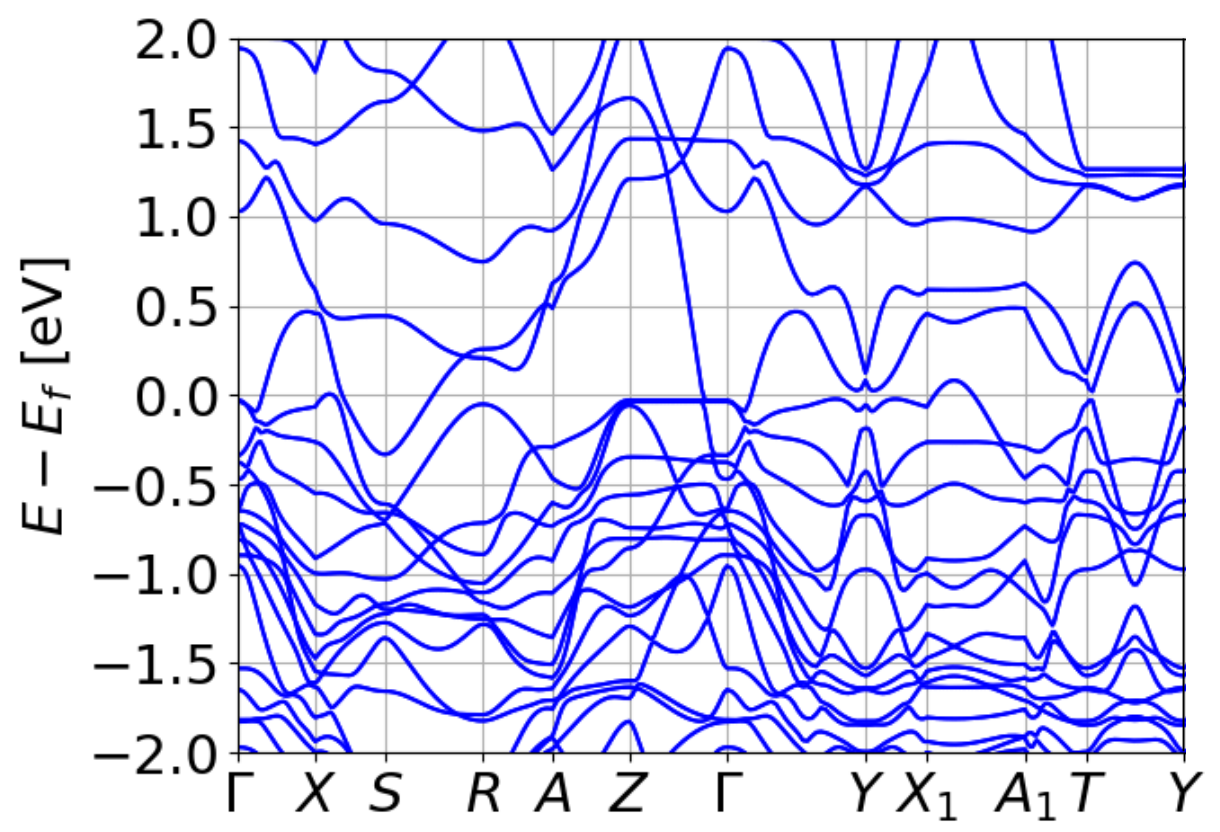

Figure 5.51: Bands structure of $\mathrm{Ni}_{5} \mathrm{Ti}$ with space group $\mathrm{Cmmm}$

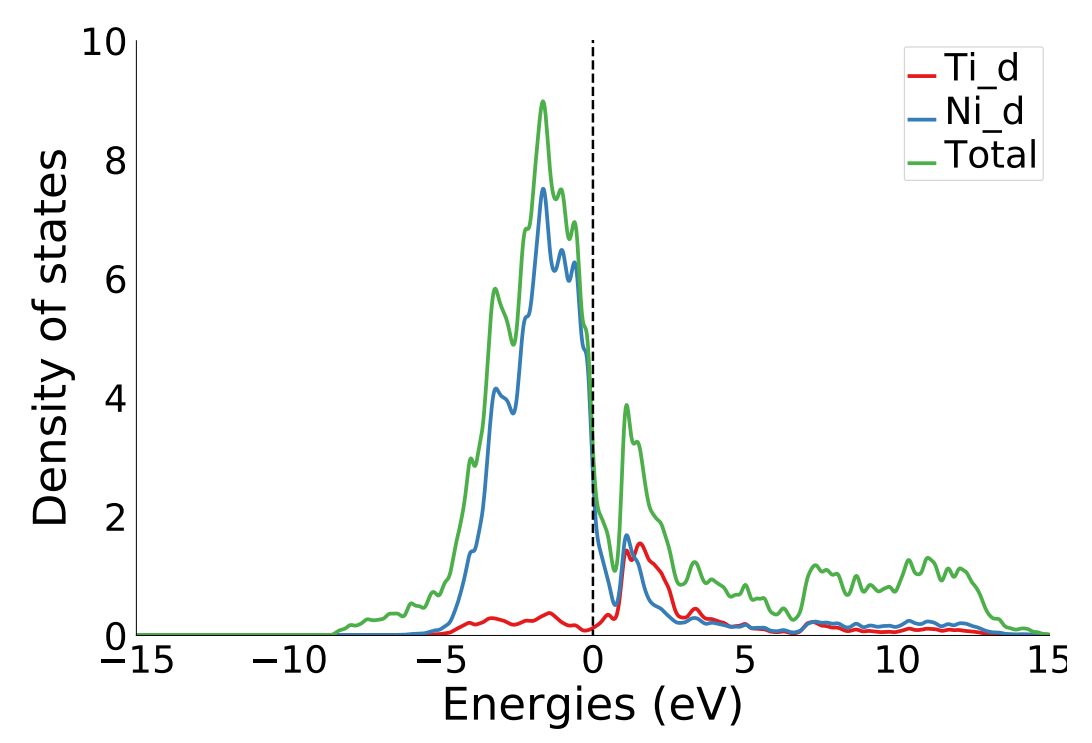

Figure 5.52: Density of states for $\mathrm{Ni}_{5} \mathrm{Ti}$ with space group $\mathrm{Cmmm}$. 
it in the lab would likely result in a combination of $\mathrm{Ni}_{5} \mathrm{Ti}_{1}, \mathrm{Ni}_{3} \mathrm{Ti}$, and pristine $\mathrm{Ti}$. However, if it could be experimentally realized, it has elastic properties which are enhanced in comparison to pristine $\mathrm{Ni}$ and Ti. Results for all other compositions which exist on the convex hull were in agreement with previous results, in particular NiTi, were both the monoclinc martensite phase and the cubic austenite phase were found. Because of the martensitic transformation in equiatomic NiTi, determing if other compositions support a cubic phase was aslo of interest. Only two other compositions, $\mathrm{Ni}_{3} \mathrm{Ti}$ and $\mathrm{Ni}_{5} \mathrm{Ti}$ support a cubic phase. For $\mathrm{Ni}_{3} \mathrm{Ti}$, this cubic phase is the ground state, so it's highly unlikely $\mathrm{Ni}_{3} \mathrm{Ti}$ supports this transformation. With $\mathrm{Ni}_{5} \mathrm{Ti}$, as the low-energy configuration is just barely on the convex hull, its unlikely $\mathrm{Ni}_{5} \mathrm{Ti}$ can be isolated to begin with.

Of further interest, a metastable monoclinc phase with space group $C l_{2} / m$ was found which has a negative Poisson ratio. Materials with this property are known as "auxetics", which means they respond to applied strain in a manner opposite to what is expected in a typical material. Additionally, a monoclinc NiTi phase with space group $P 2_{1} / m$ was found which displays negative linear compressibility. The mechanism for this effect is the well-known wine rack model. 


\section{Chapter 6}

\section{Summary and Outlook}

In this work materials prediction methods were carried out to optimize three particular properties: Magnetic moments, orbital occupations, and crystal structures. For the first two properties, a new methodology based on the metaheuristic firefly algorithm was presented. The rationale behind the development and use of this algorithm is that it lends itself well to multi-modal problems, that is material properties which support multiple energetically degenerate solutions.

For the magnetic moment case, these are frustrated magnetic systems. Two of the three test cases were of the classically frustrated picture; magnetic moments that lie on a triangular lattice. In both cases multiple degenerate configurations were found. In the case of $\mathrm{Mn}_{3} \mathrm{Pt}$, three different frustrated solutions were found, which is in agreement with theoretical arguments. For the molecular system, two degenerate configurations were found, which is also in agreement with a previous theoretical result. While the weak ferromagnetic $\mathrm{NiFi}_{2}$ is not frustrated, it is noncollinear, and the canting angle between the $\mathrm{Ni}$ manetic moments is on the order of 0.6 degrees. This result is supported both experimentally and theoretically. FA was able to locate a low-energy solution with this canting angle which also has the correct magnetocrystalline anisotropy. In all three cases, FA found results which are in good agreement, indicating that FA is well-suited to the problem of magnetic moment optimization.

In the orbital occupation optimization case, the AFM perovskite-like system $\mathrm{KCoF}_{3}$ and the $\mathrm{AFM} \mathrm{UO} \mathrm{U}_{2}$ were considered. Each was chosen based on the necessary orbital corrections to correctly describe the physics of each, for $\mathrm{KCoF}_{3}$, d-electron corrections are necessary. For $\mathrm{UO}_{2}$, f-electrons must be corrected. This was to show that FA works equally well regardless of whather d or f-electron corrections are required. For $\mathrm{KCoF}_{3}$, the low-energy state had an occupation which 
is in agreement with theoretical predictions based on octohedral crystal field splitting. In addition, the band gap and the magnetic exchange constants for this state are in agreement with the experimentally observed ground state. Similarly, the low-energy occupation for $\mathrm{UO}_{2}$ is in agreement with theoretical arguments. Also of relevance, the metastable states found are in agreement with a previous theoretical study which considered possible stable and metastable occupancies. While any searcher algorithm must yield results which are in agreement with experiment for any known system, what is striking about the use of FA for magnetic moment optimization is the multitude of metastable solutions which were found using the search. Most of this metastable states only differed in the sub-meV range, which conclusively demonstrates the necessity for search methods in magnetic systems. Thus, FA is well-suited to multi-modal optimization problems in real material systems. While the beauty of metaheuristic algorithms are their lack of dependence on system specific details, analysis of symmetries of a system could be implemented to limit the physically realizable states, as this would reduce to total number of candidates which must be searched over. As each search performed with FA in this work required the evaluation of 500 candidates, such an implementation would be useful to reduce computational cost.

For structural search, the minimum hopping method was used to locate stable crystalline structures of varying stoichiometry in the binary NiTi system. As the shape-memory effect in equiatomic NiTi has been well-studied, both theoretical and experimental results for this composition are well-known. The stable structures found using MHM for this composition are in agreement with what is previously known. For the other compositions on the convex hull, $\mathrm{Ni}_{3} \mathrm{Ti}$ is in complete agreement with previous results, a new low-energy structure with tetragonal symmetry is found for $\mathrm{NiTi}_{2}$, and a brand new orthorhombic structure is found for $\mathrm{Ni}_{5} \mathrm{Ti}$. 


\section{Bibliography}

[1] Jeremy P. Allen and Graeme W. Watson. "Occupation matrix control of $\mathrm{d}$ - and f-electron localisations using DFT + U'. In: Phys. Chem. Chem. Phys. 16 (39 2014), 21016-21031.

[2] Jeremy P. Allen and Graeme W. Watson. "Occupation matrix control of $\mathrm{d}$ - and f-electron localisations using DFT + U'. In: Phys. Chem. Chem. Phys. 16 (39 2014), 21016-21031.

[3] B. Amadon, F. Jollet, and M. Torrent. " $\gamma$ and $\beta$ cerium: LDA + U calculations of ground-state parameters”. In: Phys. Rev. B 77 (15 Apr. 2008), 155104.

[4] Maximilian Amsler and Stefan Goedecker. "Crystal structure prediction using the minima hopping method". In: The Journal of Chemical Physics 133.22 (2010), 224104.

[5] V. I. Anisimov and O. Gunnarsson. "Density-functional calculation of effective Coulomb interactions in metals". In: Phys. Rev. B 43 (10 Apr. 1991), 7570-7574.

[6] V I Anisimov et al. "First-principles calculations of the electronic structure and spectra of strongly correlated systems: dynamical mean-field theory”. In: Journal of Physics: Condensed Matter 9.35 (Sept. 1997), 73597367.

[7] Vladimir I Anisimov, F Aryasetiawan, and A I Lichtenstein. "First-principles calculations of the electronic structure and spectra of strongly correlated systems: theLDA+Umethod". In: Journal of Physics: Condensed Matter 9.4 (Jan. 1997), 767-808.

[8] Guillermo Avenda no-Franco and Aldo H. Romero. "Firefly Algorithm for Structural Search". In: Journal of Chemical Theory and Computation 12.7 (2016). PMID: 27232694, 3416-3428. 
[9] Ray Baughman et al. "Negative Poisson's ratios as a common feature of cubic metals". In: 392 (Mar. 1998), 362-365.

[10] Lowell W. Beineke, Ortrud R. Oellermann, and Raymond E. Pippert. "The average connectivity of a graph". In: Discrete Math. 252.1 (2002), 31-45. ISSN: 0012-365X.

[11] Kamel Benyelloul and Hafid Aourag. "Elastic constants of austenitic stainless steel: Investigation by the first-principles calculations and the artificial neural network approach". In: Computational Materials Science 67 (2013), 353-358. ISSN: 0927-0256.

[12] P. E. Blöchl. "Projector augmented-wave method". In: Phys. Rev. B 50 (24 Dec. 1994), 17953-17979.

[13] Eric Bousquet and Nicola Spaldin. "J dependence in the LSDA+U treatment of noncollinear magnets”. In: Phys. Rev. B 82 (22 Dec. 2010), 220402.

[14] D.J. Breed, K. Gilijamse, and A.R. Miedema. "Magnetic properties of K2CoF4 and Rb2CoF4; two-dimensional Ising antiferromagnets". In: Physica 45.2 (1969), 205-216. ISSN: 0031-8914.

[15] R. Caciuffo et al. "Magnetic excitations and dynamical Jahn-Teller distortions in $\mathrm{UO}_{2}$ ". In: Phys. Rev. B 59 (21 June 1999), 13892-13900.

[16] Andrew B. Cairns and Andrew L. Goodwin. "Negative linear compressibility". In: Phys. Chem. Chem. Phys. 17 (32 2015), 20449-20465.

[17] Andrew B. Cairns et al. "Giant negative linear compressibility in zinc?dicyanoaurate". In: Nature Materials 12.3 (2013), 212-216. ISSN: 1476-4660.

[18] V. H. Carneiro, J. Meireles, and H. Puga. "Auxetic materials - A review”. In: Materials Science-Poland 31.4 (Oct. 2013), 561-571. ISSN: 2083-134X.

[19] Anne M. Chaka et al. "Density-functional theory investigation of oxidative corrosion of UO2". In: Computational and Theoretical Chemistry 987 (2012). Applications of computational chemistry in materials science and materials chemistry, 90-102. ISSN: 2210-271X.

[20] Matteo Cococcioni. “The LDA + U Approach : A Simple Hubbard Correction for Correlated Ground States". In: 2012.

[21] Aron J. Cohen, Paula Mori-Sánchez, and Weitao Yang. "Fractional spins and static correlation error in density functional theory". In: The Journal of Chemical Physics 129.12 (2008), 121104. 
[22] Aron J. Cohen, Paula Mori-Sánchez, and Weitao Yang. "Insights into Current Limitations of Density Functional Theory". In: Science 321.5890 (2008), 792-794. ISSN: 0036-8075.

[23] A H Cooke, K A Gehring, and R Lazenby. "The magnetic properties of NiF 2”. In: Proc. Phys. Soc. 85.5 (1965), 967.

[24] John Dagdelen et al. "Computational prediction of new auxetic materials". In: Nature Communications 8.1 (2017), 323. ISSN: 2041-1723.

[25] Elbio Dagotto. "Complexity in Strongly Correlated Electronic Systems". In: Science 309.5732 (2005), 257-262. ISSN: 0036-8075.

[26] D.M. Daven et al. "Structural optimization of Lennard-Jones clusters by a genetic algorithm”. In: Chemical Physics Letters 256.1 (1996), 195-200. ISSN: 0009-2614.

[27] Boris Dorado et al. "DFT +U calculations of the ground state and metastable states of uranium dioxide". In: Phys. Rev. B 79 (23 June 2009), 235125.

[28] Boris Dorado et al. "Stability of oxygen point defects in $\mathrm{UO}_{2}$ by firstprinciples DFT $+U$ calculations: Occupation matrix control and JahnTeller distortion”. In: Phys. Rev. B 82 (3 July 2010), 035114.

[29] Ken E Evans. "Auxetic polymers: a new range of materials". In: Endeavour 15.4 (1991), 170-174. ISSN: 0160-9327.

[30] Marcelo do Amaral Ferreira, Marco AntÃ'nio Luersen, and Paulo CÃ Borges. "Nickel-titanium alloys: a systematic review". en. In: Dental Press Journal of Orthodontics 17 (June 2012), 71-82. ISSN: 2176-9451.

[31] Alessio Filippetti and Nicola A. Spaldin. "Self-interaction-corrected pseudopotential scheme for magnetic and strongly-correlated systems". In: Phys. Rev. B 67 (12 Mar. 2003), 125109.

[32] Romain Gaillac, Pluton Pullumbi, and François-Xavier Coudert. "ELATE: an open-source online application for analysis and visualization of elastic tensors". In: Journal of Physics: Condensed Matter 28.27 (May 2016), 275201.

[33] Romain Gaillac, Pluton Pullumbi, and François-Xavier Coudert. "ELATE: an open-source online application for analysis and visualization of elastic tensors”. In: Journal of Physics: Condensed Matter 28.27 (2016), 275201.

[34] Ruben Gatt, Roberto Caruana-Gauci, and Joseph Grima. "Negative linear compressibility: Giant response". In: 12 (Mar. 2013), 182-3. 
[35] Hua Y. Geng et al. "Interplay of defect cluster and the stability of xenon in uranium dioxide from density functional calculations". In: Phys. Rev. B 82 (9 Sept. 2010), 094106.

[36] Antoine Georges et al. "Dynamical mean-field theory of strongly correlated fermion systems and the limit of infinite dimensions". In: Rev. Mod. Phys. 68 (1 Jan. 1996), 13-125.

[37] Stefan Goedecker. "Minima hopping: An efficient search method for the global minimum of the potential energy surface of complex molecular systems”. In: The Journal of Chemical Physics 120.21 (2004), 9911-9917.

[38] A.A. Golestaneh and J.M. Carpenter. "Study of the martensitic transformation in shape-memory nitinol alloy by time-of-flight neutron diffraction techniques". In: Acta Metallurgica et Materialia 38.7 (1990), 1291-1305. ISSN: 0956-7151.

[39] Xavier Gonze et al. "Recent developments in the ABINIT software package". In: Computer Physics Communications 205 (2016), 106-131.

[40] X Gonze et al. "A brief introduction to the ABINIT software package". In: Zeitschrift für Kristallographie.(Special issue on Computational Crystallography.) 220 (2005), 558-562.

[41] X. Gonze et al. "ABINIT: First-principles approach to material and nanosystem properties". In: Computer Physics Communications 180.12 (2009), 2582-2615. ISSN: 0010-4655.

[42] X. Gonze et al. "First-principles computation of material properties: the \{ABINIT\} software project". In: Computational Materials Science 25.3 (2002), 478-492. ISSN: 0927-0256.

[43] G Greaves et al. "Poisson's ratio and modern materials". In: 10 (Nov. 2011), 986.

[44] Joseph Grima, Andrew Alderson, and Kenneth E. Evans. "Negative Poisson's ratio from rotating rectangles". In: 10 (Jan. 2004), 137-145.

[45] Stefan Grimme. "Semiempirical hybrid density functional with perturbative second-order correlation”. In: The Journal of Chemical Physics 124.3 (2006), 034108.

[46] K. Held et al. "Realistic investigations of correlated electron systems with LDA + DMFT”. In: physica status solidi (b) 243.11 (2006), 2599-2631. 
[47] David K. Hoffman, Richard C. Raffenetti, and Klaus Ruedenberg. "Generalization of Euler Angles to N-Dimensional Orthogonal Matrices". In: Journal of Mathematical Physics 13.4 (1972), 528-533.

[48] T M Holden et al. "Excitations in KCoF3-I. Experimental". In: Journal of Physics C: Solid State Physics 4.14 (Nov. 1971), 2127-2138.

[49] Xiangyang Huang et al. "Lattice instabilities of cubic NiTi from first principles". In: Phys. Rev. B 65 (1 Dec. 2001), 014108.

[50] M. T. Hutchings et al. "Neutron and Optical Investigation of Magnons and Magnon-Magnon Interaction Effects in $\mathrm{NiF}_{2}$ ". In: Phys. Rev. B 2 (5 Sept. 1970), 1362-1373.

[51] Anubhav Jain et al. "Formation enthalpies by mixing GGA and GGA + U calculations". In: Physical Review B 84.4 (July 2011), 045115. ISSN: 1098-0121.

[52] Anubhav Jain et al. "The Materials Project: A materials genome approach to accelerating materials innovation". In: APL Materials 1.1 (2013), 011002. ISSN: 2166532X.

[53] W.M. Jenkins. "Towards structural optimization via the genetic algorithm". In: Computers \& Structures 40.5 (1991). Special Issue: Computational Structures Technology, 1321-1327. ISSN: 0045-7949.

[54] Min Ji et al. "Ultrahigh-pressure phases of $\mathrm{H}_{2} \mathrm{O}$ ice predicted using an adaptive genetic algorithm”. In: Phys. Rev. B 84 (22 Dec. 2011), 220105.

[55] François Jollet, Marc Torrent, and Natalie Holzwarth. "Generation of Projector Augmented-Wave atomic data: A 71 element validated table in the XML format". In: Computer Physics Communications 185.4 (2014), 1246-1254. ISSN: 0010-4655.

[56] Gérald Jomard et al. "Structural, thermodynamic, and electronic properties of plutonium oxides from first principles". In: Phys. Rev. B 78 (7 Aug. 2008), 075125.

[57] K. Knox. "Perovskite-like fluorides. I. Structures of $\mathrm{KMnF}_{3}, \mathrm{KFeF}_{3}, \mathrm{KNiF}_{3}$ and $\mathrm{KZnF}_{3}$. Crystal field effects in the series and in $\mathrm{KCrF}_{3}$ and $\mathrm{KCuF}_{3}$ ". In: Acta Crystallographica 14.6 (June 1961), 583-585.

[58] Dm. M. Korotin et al. "Calculation of exchange constants of the Heisenberg model in plane-wave-based methods using the Green's function approach". In: Phys. Rev. B 91 (22 June 2015), 224405. 
[59] Y. Kota, H. Tsuchiura, and A. Sakuma. "Ab-Initio Study on the Magnetic Structures in the Ordered Mn ${ }_{3} \mathrm{Pt}$ Alloy". In: IEEE T. Magn. 44.11 (Nov. 2008), 3131-3133. ISSN: 0018-9464.

[60] G. Kotliar et al. "Electronic structure calculations with dynamical meanfield theory". In: Rev. Mod. Phys. 78 (3 Aug. 2006), 865-951.

[61] E. Krén et al. "Magnetic Structures and Exchange Interactions in the MnPt System”. In: Phys. Rev. 171 (2 July 1968), 574-585.

[62] G. Kresse and J. Furthmüller. "Efficiency of ab-initio total energy calculations for metals and semiconductors using a plane-wave basis set". In: Computational Materials Science 6.1 (1996), 15-50. ISSN: 0927-0256.

[63] G. Kresse and J. Hafner. "Ab initio molecular dynamics for liquid metals". In: Phys. Rev. B 47 (1 Jan. 1993), 558-561.

[64] G. Kresse and J. Hafner. "Ab initio molecular-dynamics simulation of the liquid-metal-amorphous-semiconductor transition in germanium”. In: Phys. Rev. B 49 (20 May 1994), 14251-14269.

[65] J Kubler et al. "Density functional theory of non-collinear magnetism". In: J. Phys. F: Met. Phys. 18.3 (1988), 469.

[66] Y Kudoh et al. "Crystal structure of the martensite in Ti-49.2 at.\%Ni alloy analyzed by the single crystal X-ray diffraction method". In: Acta Metallurgica 33.11 (1985), 2049-2056. ISSN: 0001-6160.

[67] P. Larson et al. "Electronic structure of rare-earth nitrides using the LSDA+ $U$ approach: Importance of allowing $4 f$ orbitals to break the cubic crystal symmetry". In: Phys. Rev. B 75 (4 Jan. 2007), 045114.

[68] A. I. Liechtenstein, V. I. Anisimov, and J. Zaanen. "Density-functional theory and strong interactions: Orbital ordering in Mott-Hubbard insulators". In: Phys. Rev. B 52 (8 Aug. 1995), R5467-R5470.

[69] A.I. Liechtenstein et al. "Local spin density functional approach to the theory of exchange interactions in ferromagnetic metals and alloys". In: Journal of Magnetism and Magnetic Materials 67.1 (1987), 65-74. ISSN: 0304-8853.

[70] Sijie Luo et al. "Noncollinear Spins Provide a Self-Consistent Treatment of the Low-Spin State of a Biomimetic Oxomanganese Synthetic Trimer Inspired by the Oxygen Evolving Complex of Photosystem II". In: The Journal of Physical Chemistry Letters 2.20 (2011), 2629-2633. 
[71] Jian Lv et al. "Particle-swarm structure prediction on clusters". In: The Journal of Chemical Physics 137.8 (2012), 084104.

[72] Scott M. Woodley et al. "The prediction of inorganic crystal structures using a genetic algorithm and energy minimisation”. In: Phys. Chem. Chem. Phys. 1 (10 1999), 2535-2542.

[73] V. Manivannan, P. Parhi, and Jonathan W. Kramer. "Metathesis synthesis and characterization of complex metal fluoride, $\mathrm{KMF} 3(\mathrm{M}=\mathrm{Mg}, \mathrm{Zn}$, $\mathrm{Mn}, \mathrm{Ni}, \mathrm{Cu}$ and $\mathrm{Co}$ ) using mechanochemical activation". In: Bulletin of Materials Science 31.7 (Dec. 2008), 987-993. ISSN: 0973-7669.

[74] M. J. Marcinkowski, A. S. Sastri, and D. Koskimaki. "Martensitic behaviour in the equi-atomic Ni-Ti alloy”. In: The Philosophical Magazine: A Journal of Theoretical Experimental and Applied Physics 18.155 (1968), 945-958.

[75] L. M. Matarrese and J. W. Stout. "Magnetic Anisotropy of $\mathrm{NiF}_{2}$ ". In: Phys. Rev. 94 (6 June 1954), 1792-1793.

[76] Arash A. Mostofi et al. "An updated version of wannier90: A tool for obtaining maximally-localised Wannier functions". In: Computer Physics Communications 185.8 (2014), 2309-2310. ISSN: 0010-4655.

[77] M. H. Mueller and H. W. Knott. "The Crystal Structures of Ti2Cu, Ti2Ni, Ti4Ni2O, and Ti4Cu2O". In: Transactions of American Institute of Metallurgical Engineers 227 (June 1963).

[78] Taku Onishi and Yasunori Yoshioka. "The Theoretical Study on the Spin States of the Perovskite-Type $\mathrm{KCoF}_{i} \operatorname{sub}_{i} 3_{i} / \mathrm{sub}_{i}$ Solid". In: $e$-Journal of Surface Science and Nanotechnology 5 (2007), 17-19.

[79] A. Otero-de-la-Roza and Víctor Luaña. "Gibbs2: A new version of the quasi-harmonic model code. I. Robust treatment of the static data". In: Computer Physics Communications 182.8 (2011), 1708-1720. ISSN: 00104655.

[80] G. Pari, S. Mathi Jaya, and R. Asokamani. "Electronic structure and magnetism of $\mathrm{KMF}_{3}(\mathrm{M}=\mathrm{Mn}, \mathrm{Fe}, \mathrm{Co}, \mathrm{Ni})$ ". In: Phys. Rev. B 50 (12 Sept. 1994), 8166-8169.

[81] Hyowon Park, Andrew J Millis, and Chris A Marianetti. "Computing total energies in complex materials using charge self-consistent DFT+ DMFT". In: Physical Review B 90.23 (2014), 235103. 
[82] Adam Payne et al. "Firefly Algorithm Applied to Noncollinear Magnetic Phase Materials Prediction”. In: Journal of Chemical Theory and Computation 14.8 (2018). PMID: 29966084, 4455-4466.

[83] J. P. Perdew and Alex Zunger. "Self-interaction correction to densityfunctional approximations for many-electron systems". In: Phys. Rev. B 23 (10 May 1981), 5048-5079.

[84] John P. Perdew. "Density functional theory and the band gap problem". In: International Journal of Quantum Chemistry 28.S19 (1985), 497-523.

[85] John P. Perdew et al. "Atoms, molecules, solids, and surfaces: Applications of the generalized gradient approximation for exchange and correlation”. In: Phys. Rev. B 46 (11 Sept. 1992), 6671-6687.

[86] VICTOR POLO, ELFI KRAKA, and DIETER CREMER. "Electron correlation and the self-interaction error of density functional theory". In: Molecular Physics 100.11 (2002), 1771-1790.

[87] P. L. Richards. "Far-Infrared Magnetic Resonance in $\mathrm{NiF}_{2}$ ". In: Phys. Rev. 138 (6A June 1965), A1769-A1775.

[88] Shantanu Roy, Stefan Goedecker, and Vladimir Hellmann. "Bell-EvansPolanyi principle for molecular dynamics trajectories and its implications for global optimization”. In: Phys. Rev. E 77 (5 May 2008), 056707.

[89] James E. Saal et al. "Materials Design and Discovery with High-Throughput Density Functional Theory: The Open Quantum Materials Database (OQMD)". In: JOM 65.11 (Nov. 2013), 1501-1509. ISSN: 1543-1851.

[90] Joseph E. Sarneski et al. "Assembly of high-valent oxomanganese clusters in aqueous solution. Redox equilibrium of water-stable Mn3O44+ and Mn2O23+ complexes". In: Journal of the American Chemical Society 112.20 (1990), 7255-7260.

[91] Dong-Kyun Seo. "Self-interaction correction in the LDA + U method". In: Phys. Rev. B 76 (3 July 2007), 033102.

[92] M.J. van Setten et al. "The PseudoDojo: Training and grading a 85 element optimized norm-conserving pseudopotential table". In: Computer Physics Communications 226 (2018), 39-54. ISSN: 0010-4655.

[93] David J. Singh and Dimitris A. Papaconstantopoulos. Electronic Structure and Magnetism of Complex Materials. 1st ed. Vol. 54. Springer-Verlag Berlin Heidelberg, 2003. ISBN: 9783540433828. 
[94] Tatiana Sobottka Spini et al. "Transition temperature range of thermally activated nickel-titanium archwires". In: Journal of applied oral science : revista FOB 22.2 (Apr. 2014), 109-117. ISSN: 1678-7765.

[95] J. Strempfer et al. "Magnetic properties of transition metal fluorides $M \mathrm{~F}_{2}$ ( $M=\mathrm{Mn}, \mathrm{Fe}, \mathrm{Co}, \mathrm{Ni})$ via high-energy photon diffraction". In: Phys. Rev. B 69 (1 Jan. 2004), 014417.

[96] A. Teklu et al. "Single-crystal elastic constants of Fe-15Ni-15Cr alloy". In: Metallurgical and Materials Transactions A 35.10 (Oct. 2004), 31493154. ISSN: 1543-1940.

[97] A Togo and I Tanaka. "First principles phonon calculations in materials science”. In: Scr. Mater. 108 (Nov. 2015), 1-5.

[98] Dragan Toprek, Jelena Belosevic-Cavor, and Vasil Koteski. "Ab initio studies of the structural, elastic, electronic and thermal properties of NiTi2 intermetallic". In: Journal of Physics and Chemistry of Solids 85 (2015), 197-205. ISSN: 0022-3697.

[99] Marc Torrent et al. "Implementation of the projector augmented-wave method in the $\{$ ABINIT $\}$ code: Application to the study of iron under pressure". In: Computational Materials Science 42.2 (2008), 337-351. ISSN: 0927-0256.

[100] Fabien Tran et al. "Hybrid exchange-correlation energy functionals for strongly correlated electrons: Applications to transition-metal monoxides". In: Phys. Rev. B 74 (15 Oct. 2006), 155108.

[101] S Q Wu et al. "An adaptive genetic algorithm for crystal structure prediction". In: Journal of Physics: Condensed Matter 26.3 (Dec. 2013), 035402 .

[102] Xifan Wu, David Vanderbilt, and D. R. Hamann. Systematic treatment of displacements, strains, and electric fields in density-functional perturbation theory. July 2005.

[103] S.-W. Yu et al. " $f$ - $f$ origin of the insulating state in uranium dioxide: $\mathrm{X}$-ray absorption experiments and first-principles calculations”. In: Phys. Rev. B 83 (16 Apr. 2011), 165102.

[104] G. A. Yurko, J. W. Barton, and J. G. Parr. "The crystal structure of $\mathrm{Ti}_{2} \mathrm{Ni}$ ". In: Acta Crystallographica 12.11 (Nov. 1959), 909-911. 
[105] J. Zaanen, C. Westra, and G. A. Sawatzky. "Determination of the electronic structure of transition-metal compounds: $2 \mathrm{p}$ x-ray photoemission spectroscopy of the nickel dihalides". In: Phys. Rev. B 33 (12 June 1986), 8060-8073.

[106] Zhao-Yi Zeng et al. "Lattice dynamics and phase transition of NiTi alloy". In: Solid State Communications 149.47 (2009), 2164-2168. ISSN: 00381098. 